\title{
The Interaction of Hydrogen with Deep Level Defects in Silicon
}

\author{
R. Jones, B. J. Coomer, J. P. Goss, B. Hourahine, and A. Resende \\ School of Physics, Exeter University, Stocker Road, Exeter, EX4 4QL, UK
}

\section{Table of Contents}

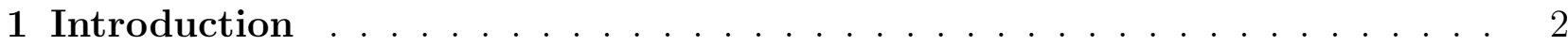

2 Theoretical Techniques ....................... 5

3 Interstitial Hydrogen . . . . . . . . . . . . . . . . . . . . . . 10

4 The $\mathbf{H}_{2}^{*}$ defect . . . . . . . . . . . . . . . . . . . . 12

4.1 Infra-Red Absorption Studies . . . . . . . . . . . . . . . . . 12

5 Hydrogen-vacancy and multi-vacancy defects . . . . . . . . . . . . . . 14

5.1 Vacancy-hydrogen complexes . . . . . . . . . . . . . . . . 15

$5.2 \mathrm{VH}_{m}$ defects in silicon . . . . . . . . . . . . . . . . 16

$5.3 \mathrm{~V}_{n} \mathrm{H}_{m}$ defects in silicon . . . . . . . . . . . . . . . . . 19

6 Self-interstitial-hydrogen complexes in silicon and germanium . . . . . . . . 21

6.1 Experimental work on $\mathrm{IH}_{2}$ defects . . . . . . . . . . . . . . . . . . . 21

6.2 The silicon self-interstitial . . . . . . . . . . . . . . . . . 22

6.3 Interstitial complexes with one hydrogen atom . . . . . . . . . . . . . 23

6.4 Di-hydrogenated split interstitials . . . . . . . . . . . . . . . . . 23

$7 \mathbf{H}_{2}$ molecules . . . . . . . . . . . . . . . . . . . . . . . 24

7.1 Experimental background . . . . . . . . . . . . . . . 26

7.2 Theoretical treatment of the hydrogen molecule in $\mathrm{Si}$. . . . . . . . . . . . . 28

7.3 Molecular diffusion barriers . . . . . . . . . . . . . . . . 32

7.4 Complexes of oxygen with hydrogen molecules . . . . . . . . . . . . . . 33

7.5 Other $\mathrm{O}-\mathrm{H}$ complexes in silicon . . . . . . . . . . . . . . . . 36

8 Evolution of $\mathbf{H}$ species in $\mathbf{S i} \ldots \ldots \ldots \ldots \ldots$

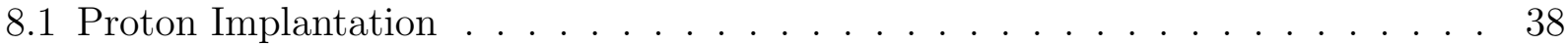

8.2 High temperature in-diffusion . . . . . . . . . . . . . . . . . . . . . . . . . . . . . . . . . . . . . 40

8.3 Plasma Treatments . . . . . . . . . . . . . . . . . . . . . . . . . . . . . . . . 43

8.4 Wet etching . . . . . . . . . . . . . . . . . . 45

9 Transition metal-hydrogen defects . . . . . . . . . . . . . . . . . . . 45

9.1 Substitutional transition-metal defects . . . . . . . . . . . . . . . . . . . . . . . . . . . . . . . . .

9.2 Modelling deep-level TM centres . . . . . . . . . . . . . . . . . . . 50

9.3 Theoretical Results . . . . . . . . . . . . . . . . . 54

$\mathrm{Au}-\mathrm{H}$ and $\mathrm{Ag}-\mathrm{H}$ defects . . . . . . . . . . . . . . . . . . . . . . . . . . . . . . . . . . . . . 54

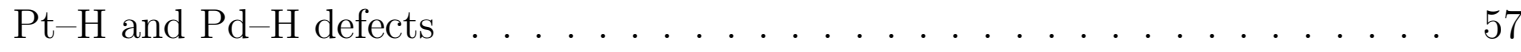

9.4 Experimental Techniques . . . . . . . . . . . . . . . . . 58

9.5 Structural properties of TM-H defects . . . . . . . . . . . . . . . . . 60

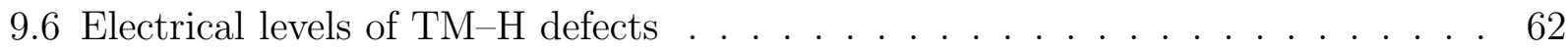

$\mathrm{Pt}-\mathrm{H}$ and $\mathrm{Pd}-\mathrm{H}$ defects . . . . . . . . . . . . . . . . . 62 
$\mathrm{AuH}$ and $\mathrm{AgH}$ defects . . . . . . . . . . . . . . . . . . . 64

9.7 Electrical passivation . . . . . . . . . . . . . . . . . 65

10 Concluding Remarks . . . . . . . . . . . . . . . . . . . . . 65

11 Acknowledgements ........................ . . 67

To be published in 'Special defects in semiconducting materials', edited by R. P. Agarwala.

\section{Introduction}

Monatomic hydrogen is a very aggressive chemical species, readily attacking $\mathrm{Si}-\mathrm{Si}$ bonds, creating an unusual bond centred defect. It reacts especially with strained bonds in vacancies and those surrounding transition metal impurities. From these considerations, it is clear that hydrogen has a great effect on centres with deep levels, as these often involve unsaturated Si atoms or possess weak bonds. However, hydrogen can also react with itself and forms several passive defects, for example hydrogen molecules, $\mathrm{H}_{2}^{*}$ defects, platelets etc. In these quiescent forms, hydrogen lies in a reservoir, but it can be extracted during processing, by heat or radiation treatments and resume its aggressive behaviour.

The monatomic impurity diffuses with a low activation energy, and is able to reach and bind with other defects. The resulting complexes are often electrically inactive but this is not always the case. The passivation of shallow impurities is well known. Less well understood is the interaction of hydrogen with neutral impurities, extended defects, and defects with deep levels such as vacancies and transition metal impurities. In some cases, gap levels are displaced or introduced and the defects can give photoluminescence lines. Hydrogen also has important catalytic effects on atomic processes, e.g., O-diffusion in $\mathrm{Si}$ and the growth of chemically vapour deposited diamond. This multitude of hydrogen related phenomena has generated extensive theoretical and experimental work. Much of this has been reviewed previously $[1,2,3]$ and here we shall concentrate on hydrogen aggregates, interstitial and vacancy hydrogen defects, and interactions with transition metal impurities, with the discussion mainly focusing on silicon.

There are four common methods used for introducing hydrogen into semiconductors. These are implantation, in-diffusion from the gas phase at high temperatures and pressures followed by a quench, via a plasma, and from the surface by the use of a wet acid etch. All are used for the study of the interactions of hydrogen with defects, but the preferred method depends on the type of experiment to be conducted. For both Fourier transform infra-red spectroscopy (FTIR) 
and electron paramagnetic resonance (EPR), high-temperature in-diffusion of hydrogen gas is particularly useful. This is because the method leads to a uniform distribution of hydrogen in the sample. FTIR observations of localised vibrational modes (LVMs) yields structural details of the bonding between hydrogen and silicon and EPR gives information on the symmetry of the defect, as well as its $g$ and hyperfine tensors. The other techniques yield inhomogeneous distributions but they can have other advantages. The latter two methods for hydrogen introduction are favoured for deep level transient spectroscopic methods (DLTS), which are used to extract the electronic levels of defects as well as their thermal stabilities.

Proton implantation at low temperatures has been used to explore the most primitive and least stable hydrogen defects. However, the techniques not only introduce very different hydrogen species but different intrinsic defects such as vacancies and interstitials. Thus these different incorporation methods, as we shall discuss below, yield more than one type of deep level defect which interact with hydrogen in different ways that are difficult to unravel. Moreover, it seems to be a general rule that deep level defects can complex with more than one hydrogen atom. For example, the vacancy can yield up to four hydrogen defects, and the interstitial two although only one has been detected so far. Similarly, transition metal impurities can react with several hydrogen atoms and there is strong evidence that Pt, for example, can bind three hydrogen atoms. All this has meant that the study of the interaction of hydrogen with deep level defects is much less developed than with shallow dopants.

Perhaps the most important properties of the deep-level hydrogen defects are their electronic levels. The principal technique used to determine these is DLTS. But, as there are always several coexisting hydrogen species, each producing levels in the band gap, the task of assigning levels to specific defects is extremely difficult. This is compounded by the difficulty that DLTS has in identifying the chemical composition and symmetry of the defect. Nevertheless, experimental groups have been resourceful. The developments of new techniques such as Laplace transform capacitance spectroscopy, the application of stress to DLTS probes, advances in defect profiling, and the correlation of DLTS with EPR and FTIR studies, has led to considerable progress. Theoretical investigations have also played an important role.

The great explosion of interest in hydrogen in semiconductors occurred at the same time as enormous progress in developments in computational theory. The widespread acceptance of 
ab initio theories coupled with a great increase in computer power, has meant that calculations and predictions are now much more reliable. Thus formation energies, equilibrium defect concentrations, structural details, vibrational modes, diffusion energies, and hyperfine parameters are routine products of these computational investigations and can be compared directly with the results of experiments. The general acceptance of the chemically challenging bond centred $\mathrm{H}$ species came so quickly because of these calculations and their explanation of the anomalous muonium species first observed in silicon and diamond. However, it is not clear whether theory will prove so useful for deep defects if their electronic levels are the prime focus. For example, $\mathrm{Au}$ appears to form at least two defects with hydrogen, and $\mathrm{Au}, \mathrm{AuH}_{1}$ and $\mathrm{AuH}_{2}$ have acceptor $(-/ 0)$ levels ${ }^{1}$ which differ only by $0.04 \mathrm{eV}$, while their donor levels differ by about $0.2 \mathrm{eV}$. Ab initio methods cannot calculate these levels to this precision: there are even difficulties in describing the band gap correctly. Thus there is a need to develop new theoretical methods, probably involving some empirical ingredient, which can address these issues. This is a real challenge and success is far from certain.

The plan of this review is as follows. We first briefly discuss the principle types of theoretical methods that have been used to describe hydrogen defects. We then discuss the results of low temperature proton implantation studies, as these have led to the most detailed information about the most primitive hydrogen species: isolated hydrogen at a bond centred site. This is a deep level defect which is bistable and has a negative- $U$, i.e. its acceptor level lies below the donor level. It is locked in the lattice at low temperatures unable to move. Upon heating the crystal, either it moves or vacancies move to it, for other defects are created and recognised from their FTIR spectra. At an early stage a dimer species called $\mathrm{H}_{2}^{*}$ is created along with vacancy-hydrogen defects. Around room temperature, a multitude of these defects denoted by $\mathrm{V}_{n} \mathrm{H}_{m}$ exist, together with the Si interstitial which is stabilised by the addition of two hydrogen atoms. These are discussed in Sections 4, 5 and 6. At higher temperatures, it has been claimed that molecules of hydrogen located at interstitial sites are produced but the evidence is not convincing. There is, however, strong experimental support for their existence, but it came from FTIR experiments carried out on material in which a different method was used to incorporate

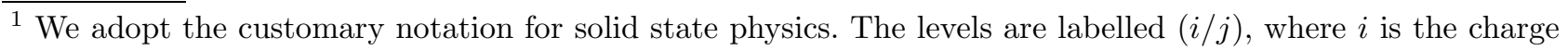
state of the defect when it is occupied and $j$ when empty.
} 
hydrogen.

Section 7 describes hydrogen complexes produced by high temperature in-diffusion treatments. Here, the dominant species in cooled specimens are hydrogen molecules. The infra-red activity and assignments of these are currently very controversial. The molecule diffuses around $100{ }^{\circ} \mathrm{C}$ although it can be trapped by many other defects, for example oxygen. These observations are important as these molecules provide a reservoir for hydrogen within the lattice, which can subsequently interfere with electrically active dopants, e.g., boron, and other defects during anneals.

Plasma treatments probably introduce all these defects together with extended ones such as platelets and voids. These will be discussed in Section 8.3. There is rather little detailed information on them at present.

Section 9 deals with the interaction of hydrogen with transition metal impurities concentrating on $\mathrm{Au}$ and Pt. There has been a great deal of recent progress in understanding these centres, and they are likely to be prototypes for many transition-metal-hydrogen defects.

There are several lattice sites within a diamond lattice which are important for a description of defects, which we shall refer to constantly. The most important of these are illustrated in Fig. 1. Among these are the bond centred $(B C)$ site which is the mid-point of the bond; the tetrahedral interstitial site, $T$, which lies at $\frac{a}{4}(\overline{1} \overline{1} \overline{1})$; the anti-bonding $(A B)$ site lying between the lattice site at (000) and the $T$ site; the $C$ site which is found at $\frac{a}{2}(100)$. Here $a$ is the lattice constant. Even if an impurity is not exactly at these sites — which often happens — we still describe it by reference to the nearest high symmetry site.

\section{Theoretical Techniques}

In treating hydrogen related defects it is essential to use a method that allows atoms to move, or relax, and permit charge to flow between atoms. This is because the energy differences between structural configurations are very sensitive to bond lengths and the charge state of the defects. This is illustrated very well by the hydrogen interstitial in Si which is bistable: the lattice location of $\mathrm{H}^{+}$is quite different from $\mathrm{H}^{-}$and in the former there is an enormous disruption to the Si lattice emphasising the need to allow for atomic relaxation.

The two standard methods of calculating the energy and structure of a defect by solving 


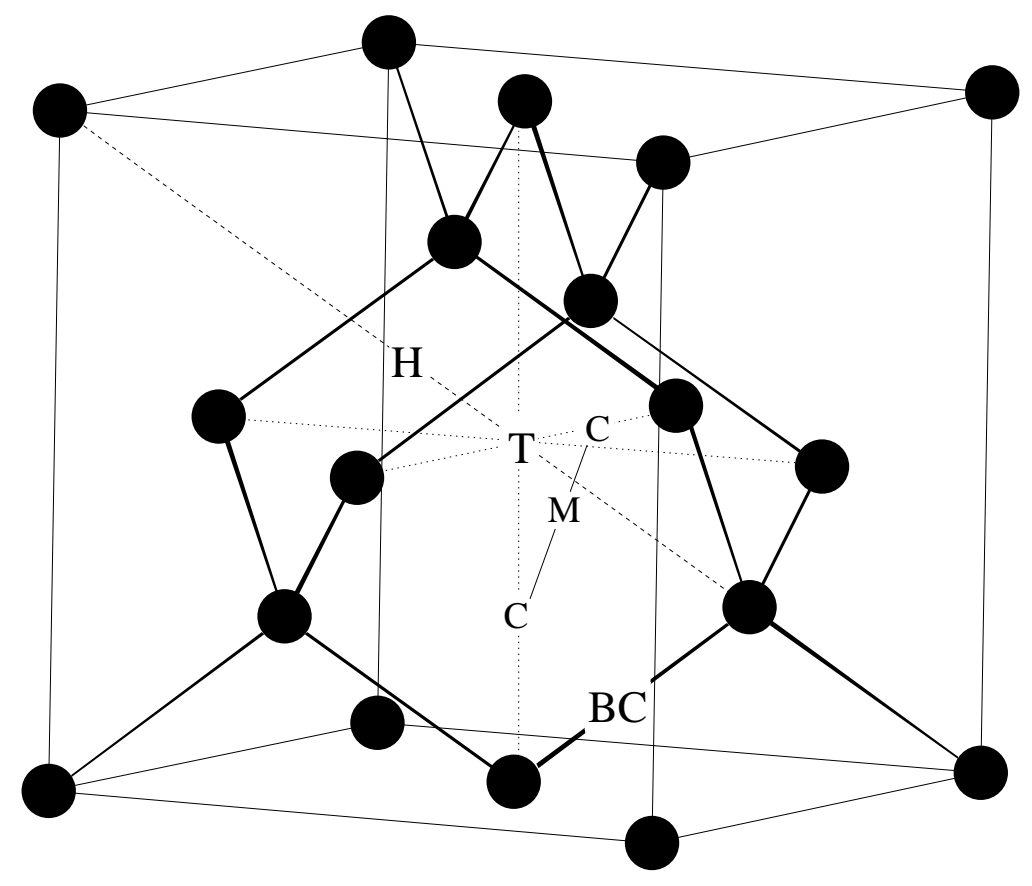

Fig. 1. A schematic of a section of diamond lattice on which the $T_{d}(T)$, hexagonal $(H)$, bond-centred $(B C), C$ and $M$ sites are indicated. The $C$ site is defined as the centre of the rhombus made up from the $T$ site and three neighbouring lattice sites, and the $M$ site is midway between two $C$ a sites.

the many-body Schrödinger equation are based on Hartree-Fock (HF) and local density functional (LDF) theories $[4,5]$. These are not free from approximations and assumptions but they have been found to be particularly useful for describing ground state molecular and crystalline structures. They are both variational procedures with HF theory assuming the wavefunction to be the variational variable whereas LDF theory, applicable only to non-degenerate ground states, takes this to be the charge density. Whereas the wavefunction depends upon the coordinates of all the electrons, the charge density is simply a function of three spatial coordinates. In the spin-polarised version, the variational variables include the magnetisation density. Both theories lead to single particle Schrödinger equations with the potential acting on an electron arising from an effective field due to all the others. Thus both require a self-consistent equation to be solved. However, there are important differences - especially in the treatment of exchange and correlation. HF theory ignores the latter and its inclusion via say Möller-Plesset [6] perturbation theory is difficult. LDF theory includes an exchange-correlation term derived from the homogeneous electron gas, but its utility in multi-atomic systems where the charge density varies rapidly is well proven.

The exchange energy in HF theory is a four-centre integral and its evaluation requires the 
computation of $\mathcal{O}\left(N^{4}\right)$ integrals, where $N$ is the basis size. This is to be contrasted with LDF theory whose exchange-correlation energy is an integral of a function of the electron density $n(\mathbf{r})$ and its evaluation requires $\mathcal{O}\left(N^{2} M\right)$ computations, where $M$ is the number of points or operations involved in estimating this integral. The development of pseudopotentials [7, 8] which remove core electrons from the problem leaving only the valence electrons to be considered, has been essential in allowing systems containing large numbers of heavy atoms to be treated. The total electron density is composed of two parts: the core and valence densities. The former is the sum of contributions from different atomic cores and is large near each nucleus but falls off rapidly to zero outside the core. The valence charge density, which although varying rapidly near the core (because of the constraints imposed by orthogonalisation), is relatively smooth around the centres of chemical bonds. Norm-conserving pseudopotentials [9] have pseudo-wavefunctions which agree exactly with those of the true atom outside the core. Thus the charge density, exchange-correlation potential and Hartree potential derived from the pseudopotential are exactly the same as those given by a full atom calculation, where all the electrons are treated explicitly. Inside the core, the pseudopotential has a repulsive part making the pseudo-wavefunctions smooth and nodeless and this makes it easier to represent them with a simple basis - although the 1s-orbital of hydrogen causes problems when a basis of plane waves is utilised. This repulsive potential is chosen so that a) the energy levels agree with the valence levels of the atom, b) the scattering phase shifts are the same for the atom and pseudo-atom and c) the core of the pseudo-atom contains the same charge as the true atom. These properties make the repulsive potential dependent on the orbital angular momentum and consequently the pseudopotential is a non-local operator. The belief is that these pseudopotentials can correctly describe different types of bonding between atoms but there is no formal proof of this.

The nature of the exchange energy in HF theory involving, as it does, the product of four orbital functions, some of which may be from the core, has made it more difficult to develop reliable pseudopotentials. It is for this reason that few calculations on large systems have been carried out and instead a multitude of approximate methods have been developed. Those with the abbreviations CNDO and MINDO ignore integrals representing matrix elements involving a pair of basis functions at distinct sites. Of course there is no compelling mathematical reason for this and it is then necessary to adjust the diagonal contributions. This is done by using 
fits to an empirical data base consisting usually of equilibrium geometries, ionisation potentials and other spectroscopic information. It is difficult to be persuaded that these methods can consistently model hydrogen defects correctly. This is especially the case for those centres far away from the situations found in the fitted data base, such as atomic configurations at saddle points or where there are unusual bonding configurations. Nevertheless, there has been an accumulation of wisdom in using these methods extending over two decades and, in the right hands, they can yield great insight into complex defects and processes. It appears, for example, that the geometries predicted by the method are often very good while the frequencies of local vibrational modes are invariably overestimated, but often by a constant amount which allows systematic scaling. Other authors [10] employ a variant of the MINDO/3 scheme, namely a cyclic-cluster scheme, in which the wavefunction is forced to be periodic within the cluster. The method seems to give surprisingly good formation and migration energies.

Nowadays, however, the emphasis is on using ab initio or, more correctly, non-empirical methods. Here there is no recourse to any experimental data base and their chief deficiencies lie as follows:

1. Their simplistic treatment of exchange-correlation;

2. The incompleteness of the basis sets used to represent the wave function or charge density;

3. The size of the cluster or supercell;

4. The difficulty in assessing whether the relaxation procedure has produced a global or local energy minimum (i.e. one corresponding to a metastable structure);

5. The convergence of the difference in the energy of the stable and saddle-point structures for the determination of migration energies;

6. The accurate location of donor and acceptor levels which control the charge states of the diffusing species.

In practice, it seems that the methods can yield excellent structures, fair vibrational modes, but the errors in defect energies are much greater. These can sometime be improved if gradient corrections are used. Electrical levels cannot easily be calculated and we describe in Section 9 an empirical correction which has proved useful.

For HF itself the method of PRDDO (partial retention of diatomic overlap), developed by Halgren and Lipscomb [11], has given useful results in a number of problems involving $\mathrm{H}$ in 
$\mathrm{Si}[2]$. Here one is judicious in the neglect of basis functions products by requiring the basis functions to be orthogonal. Only the four centre integrals involving distinct sites are neglected. In this way no parameters are necessary and the method seems efficient at giving sensible bond lengths. There are difficulties for saddle point energies — invariably too large (an effect that can happen in any theory where a seriously incomplete basis is used) and in determining vibrational modes.

Gaussian basis functions have been a popular choice for both CNDO and MINDO, as well as LDF theories. Saito and Oshiyama [12] and Jones and Briddon [13] describe methods of implementing LDF schemes using such orbitals for clusters.

In the cluster method it is necessary to terminate the cluster in such a way that the properties of the inner part are insensitive to this surface. Most workers have used H atom saturation. The idea here is to passivate surface dangling bonds which would otherwise draw charge from the inner part of the cluster to the surface and interfere greatly with the properties of the defect lying there. It is important to choose a short H-surface length as this depresses the H-bonding states below the bulk valence band top and elevates the $\mathrm{H}$-antibonding states to above the bulk conduction band. However since the host feels a confining potential from the surface $\mathrm{H}$ atoms, its valence and conduction bands are also depressed and elevated respectively, resulting in an increased band gap. However, it seems that this band gap widening does not significantly affect structural or vibrational properties, although it does lead to defect levels lying deeper in the gap than observed. It is known that small H-terminated molecules have structures and vibrational modes close to those of the bulk.

There are two ways of avoiding the use of clusters. Either one uses a Green function technique or a supercell. Kelly and Car [14], describe a Green function approach using Gaussian orbitals and incorporating pseudopotentials. The advantage of this method is that one treats an isolated defect in an infinite Si lattice. The usual problem with the Green function method is the difficulty of including the relaxation of a large number of atoms. The more atoms one relaxes, the greater the number of elements of the Green function that are required and this makes the method very time consuming.

Other LDF calculations $[15,16]$ have been carried out using plane waves and a supercell, sometimes with the molecular dynamical method of Car and Parrinello [17, 18]. These methods 
have given reasonable formation energies of defects. Here one treats the degrees of freedom of the electrons, namely the coefficients of the expansion of each orbital in terms of plane waves, together with the positions of the ions as a set of generalised coordinates in analytical dynamics. The Lagrangian for this system can then be written down together with the equations of motion. These are solved as in the usual molecular dynamical method. The ion temperature is then gradually reduced to enable the system to assume the lowest potential energy. This technique allows very large numbers of plane waves to be used in the basis.

\section{Interstitial Hydrogen}

Theoretical work on $\mathrm{H}$ in $\mathrm{Si}[19,20,21,22,23,24,15,25,26,27,28]$ has shown that $\mathrm{H}^{+}$and $\mathrm{H}^{0}$ are attracted to the lattice locations with a high charge density such as $B C$ sites and easily diffuse through the lattice with a low activation energy around $0.2-0.4 \mathrm{eV}$ [25]. In these sites there is a massive distortion of the lattice with the $\mathrm{Si}-\mathrm{Si}$ bond occupied by $\mathrm{H}$ expanding by about $40 \%$. On the other hand, $\mathrm{H}^{-}$is attracted to locations with low charge density such as an $A B$ or $T$ site. The $(0 /+)$ energy level of $\mathrm{H}_{B C}$ has been calculated to lie at $\sim E_{c}-0.2 \mathrm{eV}$ while $\mathrm{H}$ at a $T$ site has a mid-gap acceptor level $[29,30]$. The levels of the defect are then inverted and, as we shall see in Section 8.1, the neutral defect can only be metastable.

Van de Walle [31] has calculated the energies of several hydrogen defects. He finds that $\mathrm{H}_{B C}^{0}$ is higher in energy than a hydrogen molecule in the gas phase by $1.21 \mathrm{eV}$, which must then be the dissolution energy for hydrogen in silicon.

Several groups $[22,32,33,26,25,28]$ have calculated the stretch frequencies of $\mathrm{H}_{B C}$ in the positive and neutral charge states, and these are listed in Table 1.

Table 1. Calculated and experimental vibrational frequencies $\left(\mathrm{cm}^{-1}\right)$ of the bond-centred interstitial hydrogen defect in Si.

\begin{tabular}{cccl}
\hline \hline & $\mathrm{H}^{0}$ & $\mathrm{H}^{+}$ & Ref. \\
\hline Calc. & 784 & & Deák et al. $[22]$ \\
& 977 & \multirow{2}{*}{1950} & Deák et al. $[33,32]$ \\
& 800 & & DeLeo et al. $[26]$ \\
& 1945 & 2210 & Van de Walle et al. $[25]$ \\
& 1768 & 2203 & Briddon and Jones $[28]$ \\
\hline Expt. & & 1998 & Budde $[34]$ \\
\hline \hline
\end{tabular}


Experimental information on interstitial $\mathrm{H}$ has come chiefly from EPR [35], channelling [36], DLTS [29, 37, 38, 39, 40], FTIR [34] and from muon spin-polarisation [41, 19, 42]. The AA9 EPR centre attributed to bond centred hydrogen was first detected as the analogous muon centre using muon spin spectroscopy $[43,41]$. Subsequently, the same defect was detected in low temperature proton implanted $\mathrm{Si}$ [35].

The $A A 9$ centre possesses trigonal symmetry with two equivalent Si neighbours and the good agreement between the calculated and observed hyperfine parameters [44] supports its assignment to neutral $\mathrm{H}_{B C}$. The defect is metastable and only detected in the presence of band gap light. The implantation generates vacancies and possibly interstitials having deep acceptor levels leading to a Fermi-level lying below the $(0 /+)$ donor level of $\mathrm{H}_{B C}$. Thus the centre is usually positively charged and diamagnetic. However, the capture of photo-generated electrons leads to a paramagnetic species. The $A A 9$ defect disappears at different temperatures dependent on whether the annealing is carried out in the dark or in the presence of light [45]. Uniaxial stress measurement show that the activation energy for reorientation among the four $B C$ sites surrounding a Si atom is $0.43 \mathrm{eV}[46]$ - almost the same as the barrier to long range diffusion of $\mathrm{H}$ introduced into $\mathrm{Si}$ at high temperature [47].

Annealing studies show that the loss of the $A A 9$ centre around $\sim 200 \mathrm{~K}$ is correlated with the loss of the E3' DLTS level, at $E_{c}-0.16 \mathrm{eV}$, thus demonstrating that the latter is the $(0 /+)$ level of $\mathrm{H}_{B C}$. The signal can be recovered by injection of holes, implying that the loss is due to a charge state change to $\mathrm{H}^{-}$, presumably located at a $T$ site. Interestingly, Irmscher [37] found that the number of E3' centres roughly equals the number of protons implanted at low temperature and thus most end up at $B C$ sites. The same result has come from channelling [36].

The $1998 \mathrm{~cm}^{-1}$ IR absorption band detected in low temperature $\mathrm{H}$ implanted Si, and first observed by Stein [48], has recently been assigned to $\mathrm{H}_{B C}^{+}$[34]. The annealing behaviour of the $A A 9$ centre, both in the dark and under illumination, follows closely that of the $1998 \mathrm{~cm}^{-1} \mathrm{IR}$ absorption band even though the defects are in different charge states [34]. This mode has a very large effective charge of $3 e$. Unfortunately, the signal is broad $\left(\sim 2 \mathrm{~cm}^{-1}\right)$ and no satellite lines due to ${ }^{28} \mathrm{Si}-\mathrm{H}-{ }^{30} \mathrm{Si}$ combinations could be resolved whose intensities would provide irrefutable evidence that $\mathrm{H}$ is attached to two equivalent Si atoms. Nevertheless, the theoretical calculations in Table 1 are in reasonable agreement with the assignment. Curiously, no IR active modes have 
been found that could be attributed to $\mathrm{H}^{0}$ and $\mathrm{H}^{-}$, suggesting that either their effective charges or frequencies are very low.

Annealing at $\sim 200 \mathrm{~K}$ leads to the disappearance of the $1998 \mathrm{~cm}^{-1}$ band due to $\mathrm{H}_{B C}^{+}$and the creation of modes assigned to $\mathrm{H}_{2}^{*}, \mathrm{~V}_{2} \mathrm{H}$ and $\mathrm{VH}_{2}$ defects [34]. Interestingly, no Si-interstitialhydrogen defects are reported. Thus most protons implanted at low temperatures form $\mathrm{H}_{B C}^{+}$ defects, and these evolve into $\mathrm{H}_{2}^{*}$, multivacancy- $\mathrm{H}$ centres and possibly $\mathrm{H}_{2}$ molecules during warm up to room temperatures. This must be contrasted with the behaviour of hydrogen introduced from the gas phase at $1200^{\circ} \mathrm{C}$, where $\mathrm{H}_{2}^{*}$ and $\mathrm{V}_{n} \mathrm{H}_{m}$ defects are not found (see Sections 7 and 8).

Although the properties of hydrogen in its neutral and positive charge state are reasonably well understood, those of $\mathrm{H}^{-}$are more controversial. DLTS experiments have been carried out on $n$-type $\mathrm{Si}$ into which $\mathrm{H}$ has been introduced by a plasma [29]. A mid-gap (-/0) level has been deduced which is in agreement with theoretical estimates [29, 30]. However, these experiments have been criticised [39]. Evidence for the $(-/ 0)$ level has recently come from muon spin resonance experiments, carried out on $n$-type $\mathrm{Si}$ [49], and which locate the $(-/ 0)$ level of $\mathrm{Mu}^{-}$at $E_{c}-0.56 \mathrm{eV}$.

\section{$4 \quad$ The $\mathbf{H}_{2}^{*}$ defect}

Proton implantation at low temperatures leads to the formation of $\mathrm{H}_{B C}^{+}$. Annealing leads to the appearance of a new species with pronounced infrared activity. Interstitial $\mathrm{H}$ diffuses rapidly around $200 \mathrm{~K}$ and is expected to complex with many defects including itself. Early theoretical calculations in diamond suggested that a close-by donor-acceptor complex consisting of $\mathrm{H}_{B C}-\mathrm{H}_{A B}$ as shown in Fig. 2 was particularly stable [50]. Subsequent work indicated that such defects were stable in silicon $[51,16]$ (about $0.2-0.4 \mathrm{eV}$ higher in energy than $\mathrm{H}_{2}$ molecules) and germanium [52].

\subsection{Infra-Red Absorption Studies}

IR absorption studies on proton implanted silicon [53] revealed four LVMs at 2062, 1838, 1599 and $817 \mathrm{~cm}^{-1}$, now assigned to $\mathrm{H}_{2}^{*}$. These anneal out together around $180^{\circ} \mathrm{C}$ showing that they arise from the same defect. Uniaxial stress measurements demonstrate that the defect has $C_{3 v}$ 
symmetry. There are two distinct modes due to the H-D species of the defect implying that the two $\mathrm{H}$ atoms in $\mathrm{H}_{2}^{*}$ are inequivalent. The weak line at $1599 \mathrm{~cm}^{-1}$ is an overtone of the $E$-mode at $817 \mathrm{~cm}^{-1}$.

Cluster calculations [53] of the modes of the defect and their isotope shifts are consistent with this model and reproduce the small shifts in the H-stretch modes when the second $\mathrm{H}$ atom is replaced by deuterium. This implies that the distance between the $\mathrm{H}$ atoms, which controls their dynamical coupling, is roughly correct and rules out earlier suggestions as to the origin of the lines [54]. The calculated and experimental frequencies are compared in Table 2. The 2062 and $1838 \mathrm{~cm}^{-1}$ LVMs represent $\mathrm{Si}-\mathrm{H}_{B C}$ and $\mathrm{Si}-\mathrm{H}_{A B}$ stretch modes respectively. No satellite lines due to $\mathrm{Si}$ isotopes have been reported. It has been suggested that $\mathrm{H}_{2}^{*}$ is both mobile [51], and also unstable under illumination [55]. It is not believed to be electrically active. Van de Walle [31] finds the dissociation energy of $\mathrm{H}_{2}^{*}$ into two $\mathrm{H}_{B C}^{0}$ defects to be $1.2 \mathrm{eV}$.

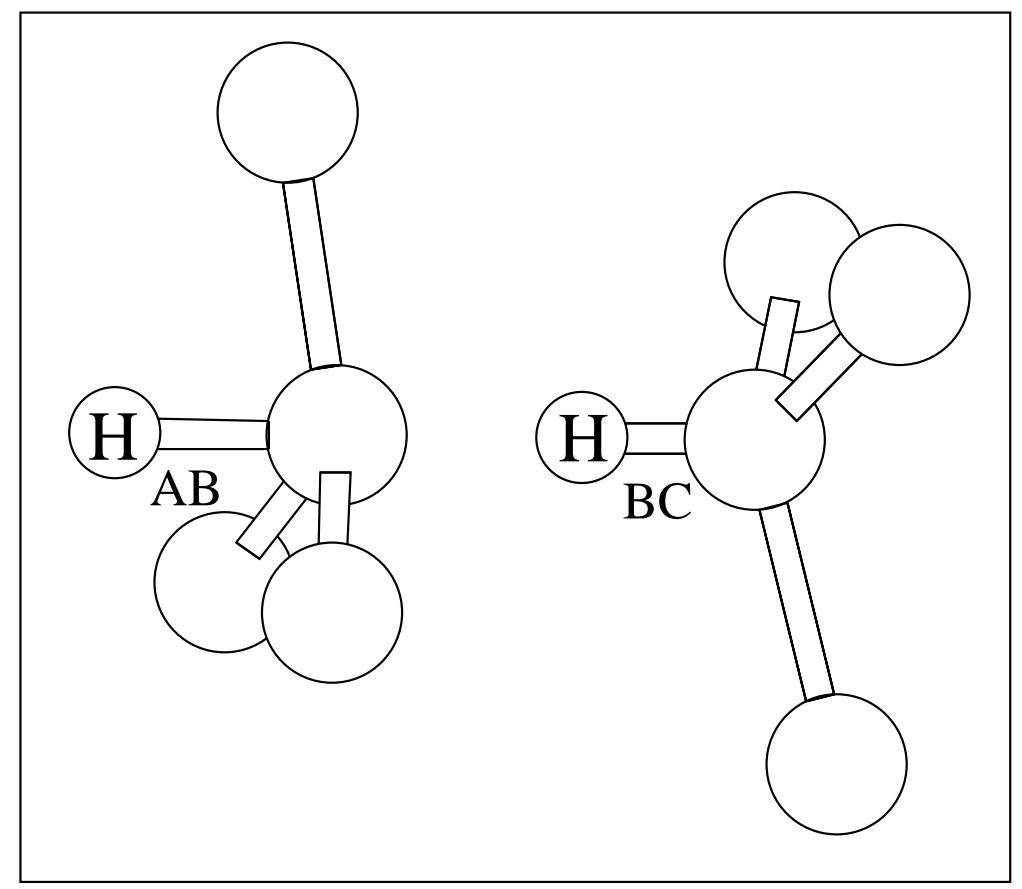

Fig. 2. Schematic figure of the $\mathrm{H}_{2}^{*}$ defect, showing the hydrogen atoms at the near bond centre $(B C)$ and anti-bonding $(A B)$ sites in the diamond lattice.

$\mathrm{H}_{2}^{*}$ has also been shown to exist in Ge. In a combined theoretical and experimental paper, Budde et al. [52] found that proton implantation in germanium produced a series of IR active lines at 765, 1499, 1774 and $1989 \mathrm{~cm}^{-1}$ with correlated intensities due to a trigonal defect. The $765 \mathrm{~cm}^{-1}$ mode was two dimensional, with an overtone at $1499 \mathrm{~cm}^{-1}$, and primarily involving 
Table 2. Calculated and measured frequencies of $\mathrm{H}_{2}^{*}$ in silicon $\left(\mathrm{cm}^{-1}\right)$.

\begin{tabular}{ccccl}
\hline \hline \multicolumn{2}{c}{$\mathrm{H}_{B C}$} & \multicolumn{3}{c}{$\mathrm{H}_{A B}$} \\
Stretch & Wag & Stretch & Wag & Ref. \\
\hline 2070 & 220 & 1480 & 690 & Chang et al. $[56]$ \\
2164 & 612 & 1844 & 1002 & Holbech et al. $[53]$ \\
2062 & & 1838 & 817 & Experimental [53] \\
\hline \hline
\end{tabular}

the stretch of the $A B$-hydrogen atom. The 1774 and $1989 \mathrm{~cm}^{-1}$ lines are one dimensional stretch modes of the $A B$ - and $B C$-hydrogen atoms respectively.

\section{$5 \quad$ Hydrogen-vacancy and multi-vacancy defects}

The interaction of hydrogen with intrinsic point defects is currently of great interest. The perturbation in the structure and electronic levels of lattice vacancies caused by the addition of hydrogen is of fundamental importance as it has implications for our understanding of Jahn-Teller (JT) systems. Room temperature proton implantation leads, as we have discussed in Section 3, to $\mathrm{V}_{n} \mathrm{H}_{m}$ centres. It is easy to understand their formation through the interaction of the intrinsic defects created by implantation with hydrogen. However, vacancy (V) and self-interstitial (I) clusters may also be created during growth, through a variety of processing techniques or even during device operation. Although intrinsic defects are always present to some degree, their abundance can, in certain circumstances, be greatly enhanced during growth. For example, rapidly grown Si crystals tend to have a larger vacancy concentrations than slowly grown ones. Impurity doping or contamination can also alter the vacancy concentration. Processing stages such as ion implantation and surface treatments such as etching and contact deposition are also known to increase the abundance of intrinsic defects. Finally, devices employed in radiation environments suffer degradation due to the creation of critical concentrations of vacancies and interstitials. Although the monovacancy and interstitial are initially the more abundant radiation defects, the dominant centre observed in electron irradiated high-purity silicon at room temperature is the divacancy $\left(\mathrm{V}_{2}\right)[57]$. Annealing at higher temperatures leads to larger vacancy aggregates such as $V_{3}$ and $V_{4}[58,59]$. Higher order aggregates like $V_{6}$ are thought to undergo extensive reconstruction and as a result can be stable defects persisting to high temperatures [60], although this centre is yet to be identified experimentally. 
In any irradiation treatment, the primary damage must result in as many interstitials as vacancies. However, it seems that the interstitials are much less reactive with hydrogen than vacancies. Whereas there are many $\mathrm{V}_{n} \mathrm{H}_{m}$ defects already characterised, e.g., $\mathrm{VH}_{n}, n=1, . .4$, $\mathrm{V}_{2} \mathrm{H}, \mathrm{V}_{3} \mathrm{H}$ and $\mathrm{V}_{2} \mathrm{H}_{6}$, there is only one interstitial $\mathrm{H}$ defect known, namely $\mathrm{IH}_{2}$. We can speculate that this is because the interstitial has a donor level leaving it in a positive charge state in

low temperature proton implanted material, and will not complex with the dominant $\mathrm{H}_{B C}^{+}$ species. Indeed, it may only be after the creation of $\mathrm{H}_{2}^{*}$, that $\mathrm{IH}_{2}$ can be formed in detectable concentrations. However, whatever the true explanation, it certainly is the case that $\mathrm{V}_{n} \mathrm{H}_{m}$ form the dominant defects and as a result the detailed interaction of $\mathrm{H}$ with the vacancy and divacancy has received considerable attention $[61,31,62]$.

\subsection{Vacancy-hydrogen complexes}

Theoretical studies of the vacancy and divacancy centres in silicon has given rise to intense controversy concerning the exact nature of the JT distortions involved. It is now apparent that the high sensitivity of the 'reconstructed bonds' characteristic of these defects gives rise to slow convergence of structure with calculation size. The addition of hydrogen to these centres adds further subtleties to their treatment. Theoretical studies of the interaction of hydrogen with intrinsic defects have thus far been restricted to monovacancy-hydrogen and interstitialhydrogen complexes. The first have been investigated by NDDO [63], tight-binding theory [64, 65], $\mathrm{HF}[65]$ and DFT [61, 66, 67].

Experimentally, $\mathrm{VH}_{m}(m=1,2,3$, and 4$)$ complexes in Si have been studied using IRabsorption [62, 68], optical detection of magnetic resonance (ODMR) [69] and more recently by EPR [70, 71]. The FTIR experiments reveal a set of LVMs between 2222 and $2052 \mathrm{~cm}^{-1}$ which are believed to be due to $\mathrm{VH}_{m}$ complexes. The EPR studies yield the symmetry of the defects and details of the JT activity of the various charge states. In the case of the vacancy or divacancy where weak, elongated bonds and dangling bonds are available, the binding of $\mathrm{H}$ to these sites is expected to be strongly exothermic. The dissociation energy of $\mathrm{VH}_{2}$ into $\mathrm{V}$ and $\mathrm{H}_{2}$ has been calculated to be $4.0 \mathrm{eV}$ [72]. Other calculations [73] show that $\mathrm{V}_{2}^{0}$ and $\mathrm{V}_{2}^{-}$also provide active sites for $\mathrm{H}_{2}$ dissociation with associated energies of 2.5 and $2.1 \mathrm{eV}$ respectively. 


\section{2 $\quad \mathrm{VH}_{m}$ defects in silicon}

The structure of $\mathrm{VH}_{m}$ has been calculated by a variety of methods. One recent calculation [63] predicts that $\mathrm{VH}_{4}$ consists of four $\mathrm{H}$ atoms lying in $\mathrm{Si}-\mathrm{Si}$ bonds around the second coordination shell leaving the vacancy region free. However, this structure is not favoured by more sophisticated treatments. The most established model assigns the set of LVMs between 2222 and $2052 \mathrm{~cm}^{-1}$ to the stretch modes of $\mathrm{Si}-\mathrm{H}$ bonds where the $\mathrm{H}$ atoms saturate the dangling bonds of the vacancy (see Fig. 3). For the case of $\mathrm{VH}$ and $\mathrm{VH}_{2}$ the reconstruction of the remaining dangling bonds occurs. There is still some debate concerning this model $[74,75,76]$.

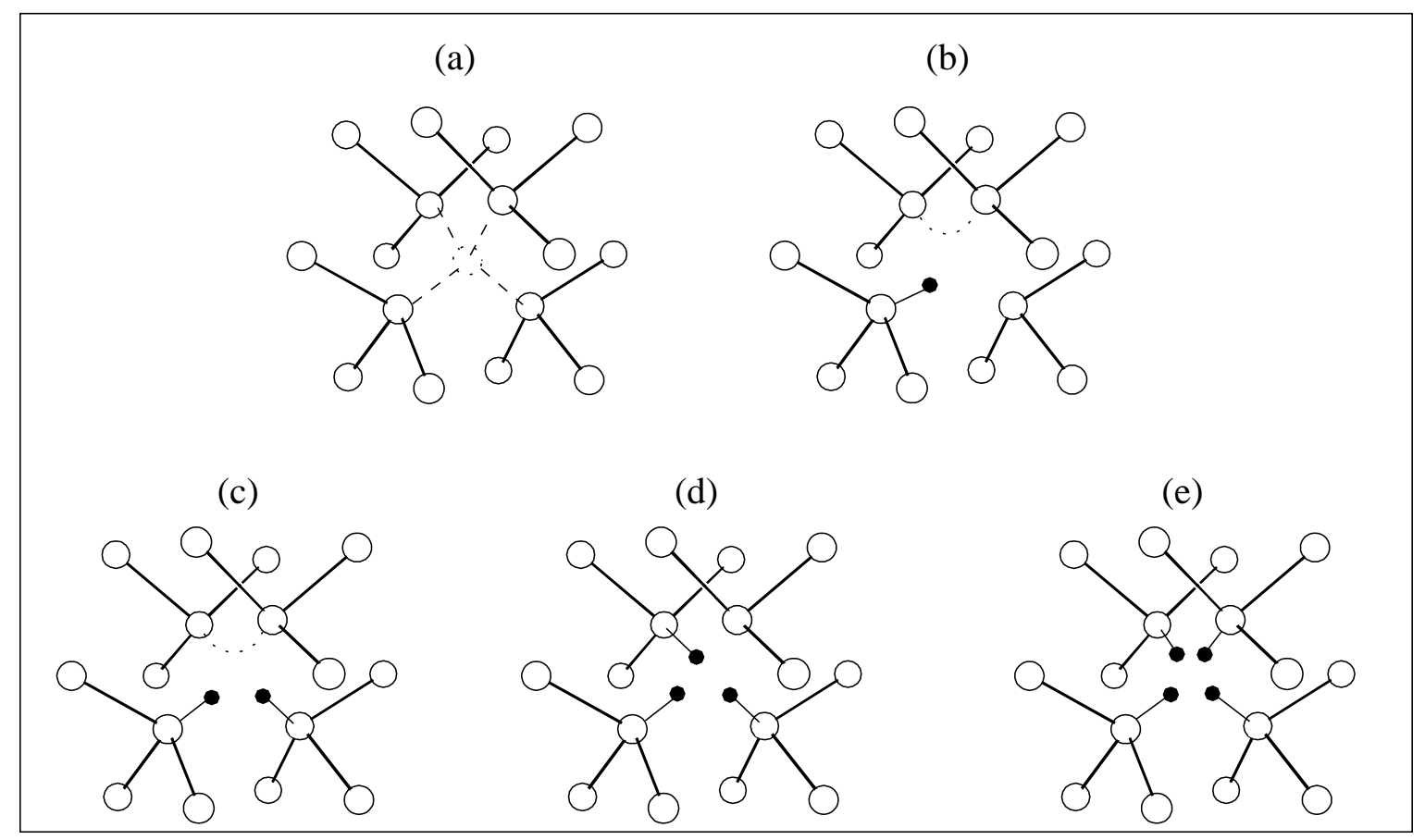

Fig. 3. The vacancy and vacancy-hydrogen structures in silicon. Dotted lines indicate weakly reconstructed bonds.

Systematic FTIR and EPR investigations of the complexes of hydrogen with native defects in silicon have provided conclusive identifications of many fundamental centres. Using isotopic substitution and annealing techniques the FTIR signals corresponding to $\mathrm{Si}-\mathrm{H}$ stretch modes of $\mathrm{VH}, \mathrm{VH}_{2}, \mathrm{VH}_{3}, \mathrm{VH}_{4}$ and $\mathrm{V}_{2} \mathrm{H}_{6}$ are assigned [34]. The assignment of two stretch modes at 2191 and $2166 \mathrm{~cm}^{-1}$ was initially made to $\mathrm{VH}_{3}[62,77]$. However, further annealing studies by the same group show that the EPR signal corresponding to $\mathrm{VH}_{3}$ anneals out at $\sim 500 \mathrm{~K}$, the temperature at which the 2191 and $2166 \mathrm{~cm}^{-1}$ LVMs first appear. Moreover, these IR lines show a greater thermal stability than those attributed to $\mathrm{VH}_{4}$. Therefore these stretch modes 
were reassigned to $\mathrm{V}_{2} \mathrm{H}_{6}$. Another pair of stretch modes at 2185 and $2155 \mathrm{~cm}^{-1}$ have since been ascribed to $\mathrm{VH}_{3}[78]$.

LDA-DFT calculations [67] using 134 atom clusters on $\mathrm{VH}_{4}$ show that the defect has tetrahedral symmetry with four $\mathrm{Si}-\mathrm{H}$ bonds of length $1.489 \AA$ pointing towards the centre of the defect (Fig. 3e). As a result of accommodating the $\mathrm{H}$ atoms the $\mathrm{Si}$ atoms are pushed away from the defect centre resulting in a $67 \%$ volume increase over the ideal vacancy. A $T_{2}$ stretch mode is observed at $2222 \mathrm{~cm}^{-1}$. The calculated (quasi-harmonic approximation) frequency is $2319 \mathrm{~cm}^{-1}$. A higher, infrared inactive mode of $A_{1}$ symmetry is calculated to lie at $2404 \mathrm{~cm}^{-1}$.

Calculations performed on $\mathrm{VH}_{3}$ in both the positive and neutral charge states predict a trigonal symmetry $\left(C_{3 v}\right)$. Neutral $\mathrm{VH}_{2}$ and $\mathrm{VH}$ are found to possess $C_{2 v}$ and $C_{1 h}$ symmetries respectively. In both cases, a weak bond was formed between a pair of the unhydrogenated Si atoms (Fig. 3b and 3c). The $\mathrm{Si}-\mathrm{H}$ stretch modes are calculated to increase with the number of $\mathrm{H}$ atoms in the vacancy $[67,32,79]$. This reflects a modest contraction of the $\mathrm{Si}-\mathrm{H}$ bond due to the repulsive $\mathrm{H} \cdots \mathrm{H}$ interaction within the vacancy. This frequency shift is also observed experimentally [62].

Table 3. Observed and calculated (quasi-harmonic) stretch frequencies $\left(\mathrm{cm}^{-1}\right)$ [67].

\begin{tabular}{lccccc}
\hline \hline & $\mathrm{VH}_{4}$ & $\mathrm{VH}_{3} \mathrm{D}$ & $\mathrm{VH}_{2} \mathrm{D}_{2}$ & $\mathrm{VHD}_{3}$ & $\mathrm{VD}_{4}$ \\
\hline Obs. & $\mathrm{N}-\mathrm{IR}, 2222$ & $2250,2224,1620$ & $2244,2225,1628,1615$ & $2236,1636,1616$ & $\mathrm{~N}-\mathrm{IR}, 1617$ \\
Cal. & 2404,2319 & $2384,2319,1677$ & $2364,2319,1690,1663$ & $2342,1705,1664$ & 1721,1664 \\
\hline \hline & $\mathrm{VH}_{3}$ & $\mathrm{VH}_{2} \mathrm{D}$ & $\mathrm{VHD}_{2}$ & $\mathrm{VD}_{3}$ & \\
\hline Obs. & 2185,2155 & & & \\
Cal. & 2318,2256 & $2298,2256,1632$ & $2277,1646,1619$ & \\
\hline & $\mathrm{VH}_{2}$ & $\mathrm{VHD}$ & $\mathrm{VD}_{2}$ & & \\
\hline Obs. & 2144,2121 & 2134,1555 & 1564,1547 & \\
Cal. & 2316,2267 & 2292,1641 & 1658,1625 & \\
\hline \hline
\end{tabular}

Table 3 compares the calculated quasi-harmonic vibrational frequencies of vacancy-hydrogen complexes with those determined experimentally [62]. Other groups have found similar modes 
$[32,79]$. The calculated modes consistently overestimate the LVMs by between 4 and $8 \%$. The reason for the overestimate lies partly in the neglect of anharmonic terms. The effect of anharmonicity was tested by finding the energy necessary to displace the atoms in each normal coordinate and then solving the Schrödinger equation for the oscillator using the shooting method [80]. The resultant symmetric frequency of $\mathrm{VH}_{2}$ was found increase by only $2 \mathrm{~cm}^{-1}$. The asymmetric stretch frequency however was found to increase by $47 \mathrm{~cm}^{-1}$ reducing the splitting of the modes to $4 \mathrm{~cm}^{-1}$. This is now a substantial underestimate of the observed $23 \mathrm{~cm}^{-1}$ splitting. The difference is proposed to arise from the neglect of coupling effects between stretch and wag modes in the theoretical treatment. Increased computational power has recently allowed larger calculations to be performed [81] employing larger, 300 atom clusters. The calculated LVMs of $\mathrm{VH}_{4}$ show a significant improvement. The more realistic strain modelling and improvements to the charge density description lowers the absolute error to less than 1\%. In particular, finer treatment of the Coulombic coupling between $\mathrm{H}$ atoms appears to be responsible for the increased accuracy of the stretch modes.

VH undergoes a change in symmetry above $\sim 60 \mathrm{~K}[71]$ from $C_{1 h}$ to $C_{3 v}$. The reconstruction across the vacancy appears to reorientate rapidly between pairs of the three available dangling bond orbitals. This thermally activated reorientation is consistent with a similar effect observed in $\mathrm{V}_{2}$ [57] between 40 and $100 \mathrm{~K}$, where the symmetry changes from $C_{2 h}$ to $D_{3 d}$. The VH EPR signal broadens and disappears above $\sim 200^{\circ} \mathrm{C}$, the broadening indicating a further increase in symmetry, possibly due to rapid reorientation of the $\mathrm{H}$ atom around the vacancy.

Although the acceptor and donor levels of $\mathrm{VH}_{n}$ defects have not been published, information on the electronic activity of the centres can be gleaned from the Kohn-Sham spectra of LDF calculations. When all the dangling bonds are saturated by $\mathrm{H}$, as in $\mathrm{VH}_{4}$, the centre becomes passive. VH is likely to be an amphoteric centre giving rise to both an acceptor and donor level. Both $\mathrm{VH}_{2}$ and $\mathrm{VH}_{3}$ probably introduce single acceptor levels only.

Hydrogen forms covalent bonds with Ge with a strength of $3.6 \mathrm{eV}$ in molecular compounds compared with a Ge-Ge dissociation energy of $1.93 \mathrm{eV}$. In comparison with silicon systems then we would expect largely similar behaviour. However, the few available comparisons of $\mathrm{H}$ in $\mathrm{Si}$ and Ge suggest otherwise. For example, whilst the passivation of shallow centres by $\mathrm{H}$ in silicon is almost complete, in germanium the passivation is only partial [82]. The motivation for the 
study of the passivation of deep centres in germanium is clearly strong particularly with the recent revolution in $\mathrm{Si}_{1-x} \mathrm{Ge}_{x}$ technology.

Surprisingly, only one systematic study of $\mathrm{V}_{n} \mathrm{H}_{m}$ defects in Ge has been performed to date. IR absorption techniques are used in combination with annealing and isotopic substitution to identify the centres $[34,83]$. The only other experimental report includes an assignment of an acceptor level at $E_{v}+0.080 \mathrm{eV}$ to $\mathrm{VH}_{2}[84]$.

The relative frequencies of the vibrational stretch modes of the $\mathrm{VH}_{m}, m=2,3$, and 4 defects in germanium have been observed to be very similar to those identified in silicon with a constant factor mapping stretch frequencies onto their counterparts in germanium. The vibrational modes for $\mathrm{VH}$ have not yet been observed. There has also been some doubt as to the assignment of the stretch modes to $\mathrm{VH}_{3}$. As in silicon, the annealing data demonstrates that these modes arise from a defect which is thermally more stable than $\mathrm{VH}_{4}$ supporting their identification with $\mathrm{V}_{2} \mathrm{H}_{6}$.

The symmetry of the defects in Ge were found to be identical to their Si counterparts. The Ge- $\mathrm{H}$ bond lengths of $\mathrm{VH}_{m}, m=1,2,3$, and 4 have been calculated [83] to be 1.526, 1.536, 1.531, and 1.541 A. Again we observe the effects of repulsion between H atoms in the vacancy. In $\mathrm{VH}_{4}$ the Ge atoms move away from the vacancy by $0.32 \AA$ giving an increase in volume of $64 \%$ over the ideal vacancy.

The shifts and splittings in the Kohn-Sham levels are similar in germanium to silicon.

\section{3 $\quad \mathbf{V}_{n} \mathbf{H}_{m}$ defects in silicon}

Both the experimental and theoretical investigations of $\mathrm{V}_{n} \mathrm{H}_{m}$ complexes are in their infancy. The identification of EPR signals corresponding to $\mathrm{V}_{2} \mathrm{H}^{0}$ and $\mathrm{V}_{3} \mathrm{H}^{0}$ has been made [71]. With a multitude of possible configurations for $\mathrm{V}_{n} \mathrm{H}_{m}$, it is clear that theoretical calculations will be necessary to aid identification of the associated IR, EPR and DLTS signals. However, a serious difficulty is that many of the defects share characteristics which leave them practically indistinguishable from others. For example, it is now clear that dynamically, $\mathrm{V}_{2} \mathrm{H}_{6}$ behaves as two weakly coupled $\mathrm{VH}_{3}$ centres resulting in vibrational modes corresponding to $g$ or $u$ parity. However, only one of these sets is infra-red active and thus the numbers and disposition of FTIR lines due to $\mathrm{VH}_{3}$ are almost identical with those of $\mathrm{V}_{2} \mathrm{H}_{6}$. 
Since the pioneering EPR work performed on irradiated silicon [57], detailed theoretical agreement with experimental data on the divacancy has remained elusive. The problem hinges upon the exact nature of the ground state geometric structure. The positive and negative charge states of $V_{2}$ have been identified with the EPR spectra labelled Si-G6 and Si-G7 respectively. Although the ideal divacancy is a trigonal centre $\left(D_{3 d}\right.$ point group symmetry — see Fig. 4$)$ both spectra have overall spin $S=\frac{1}{2}$ and are found to possess $C_{2 h}$ symmetry below $40 \mathrm{~K}$. The original model put forward proposes a reconstruction of the dangling bonds by pairs across the divacancy resulting in two weak reconstructed bonds and two dangling bonds (so that $l_{b c}<l_{a b}=l_{a c}, l_{b^{\prime} c^{\prime}}<l_{a^{\prime} b^{\prime}}=l_{a^{\prime} c^{\prime}}$ in Fig. 4 , where $l_{a b}$ is the separation between the sites labelled $a$ and $b$ and so on). The distortion is a manifestation of the JT effect. This pairing distortion also offers an explanation of the effects of uniaxial stress upon the defect.
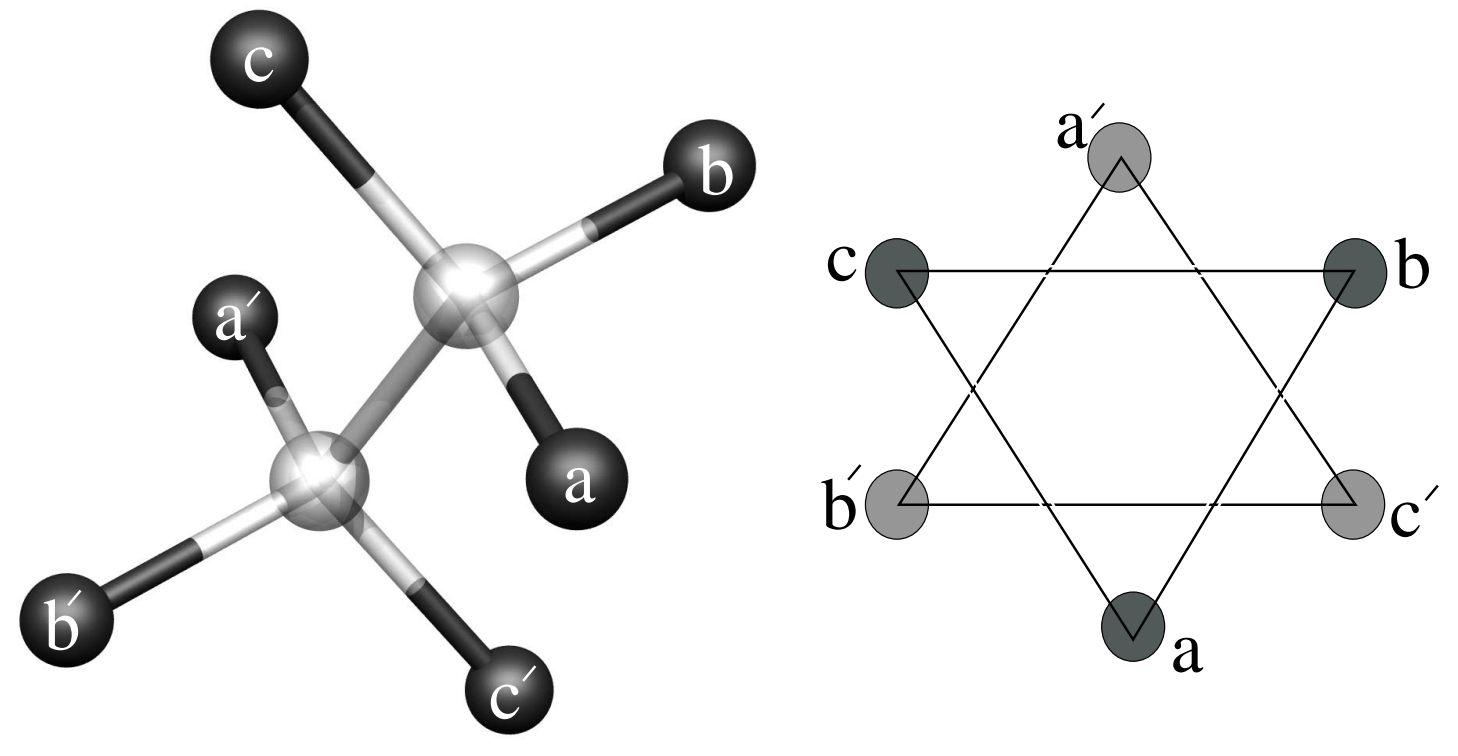

Fig. 4. Undistorted structure of the divacancy ( $D_{3 d}$ point group symmetry).

To date, none of the ab initio calculations performed on $\mathrm{V}_{2}[85,86,87]$ have confirmed the model for both charge states. The first principles calculations all involve supercells with between 64 and 216 atom sites. Saito and Oshiyama [86] find the lowest energy distortion to be inverse $\left(l_{b c}>l_{a b}=l_{a c}, l_{b^{\prime} c^{\prime}}>l_{a^{\prime} b^{\prime}}=l_{a^{\prime} c^{\prime}}\right)$ to the pairing model for the negative charge state. In this case four of the six nearest neighbour atoms move away from the defect centre towards their bonded neighbours. Although this model explains the observed spin density, it appears inconsistent with the results of the stress studies. Even using larger supercells, other calculations appear to 
agree with Oshiyama's 'resonant bonding' model [85]. Cluster-based calculations [88] currently underway, however, support the original pairing model for both charge states.

Evidently, the calculated structure of $\mathrm{V}_{2}$ must be fully reconciled with experiment before progress is made concerning the theory of $\mathrm{V}_{2} \mathrm{H}_{m}$ defects. Assuming the pairing model to be correct, the approximate structures of all but one of the centres can be unambiguously established. When adding $\mathrm{H}$ to the vacancy we simply maximise the number of reconstructed bonds in preference to the relatively high energy dangling bonds. With this procedure only $\mathrm{V}_{2} \mathrm{H}_{3}$ has more than one possible geometric configuration.

\section{Self-interstitial-hydrogen complexes in silicon and germanium}

The structure and properties of self-interstitial defects in elemental semiconductors like Si and Ge have long been of interest, but their rapid movement in $e$-irradiated material and their reactivity have prevented direct observations. Nevertheless, their trapping by other impurities or lattice defects remains highly topical, often providing a mechanism to lower the diffusion energy of the impurity (transient enhanced diffusion). It is well known that $\mathrm{C}, \mathrm{Al}$ and $\mathrm{B}$ can trap interstitials in $\mathrm{Si}$ even at temperatures as low as $77 \mathrm{~K}$, hence carbon is often deliberately incorporated in material to act as a sink for the mobile interstitial Si. It is suspected that $\mathrm{H}$ can react with these mobile self-interstitials, stabilising them and facilitating their detection by experiment. Calculations on this system have been published by a number of groups [33, 89, 90, 91].

\subsection{Experimental work on $\mathrm{IH}_{2}$ defects}

Budde et al. [90, 91], implanted silicon and germanium with protons at $\sim 100 \mathrm{~K}$. In silicon, after annealing at around room temperature, they observed LVMs at 743.1, 748.0, 1986.5 and $1989.4 \mathrm{~cm}^{-1}$. In a previous study on neutron-irradiated Si, which had been plasma treated, Xie et al. [68] had already assigned the 1986.5 and $1989.4 \mathrm{~cm}^{-1}$ modes to $\mathrm{VH}_{2}$. This however, is in conflict with later assignments (see Section 5.2). In germanium four similar modes at $700.3,705.5,1881.8$ and $1883.5 \mathrm{~cm}^{-1}$ were observed. In both materials, annealing and isotopic studies with deuterium showed that the groups of lines were due to one type of defect, with two equivalent but weakly coupled hydrogen atoms. Uniaxial stress gave the defect symmetry as $C_{2}$. These lines disappear at around $\sim 230$ and $\sim 200^{\circ} \mathrm{C}$ for $\mathrm{Si}$ and Ge respectively [34]. 


\subsection{The silicon self-interstitial}

Calculations by a number of groups $[89,92,93,94,95]$ suggest that the minimum energy structure for the self-interstitial is the $\langle 110\rangle$ split-structure with $C_{2 v}$ symmetry shown in Fig. 5a. Here two Si or Ge atoms share a single lattice site oriented along $\langle 110\rangle$. The defect has two, almost degenerate, fully occupied Kohn-Sham levels deep in the gap. There are also two unoccupied levels but the donor and acceptor levels of the centre has not been calculated (see Section 9.2). An alternative configuration for the interstitial is similar to the structure of the carbon interstitial $\left(\mathrm{C}_{i}\right)[96,97]$ and consists of two atoms sharing a lattice site along $\langle 100\rangle$. The equivalence of the two $\mathrm{Si}$ atoms in the defect core for the ideal defect results in $D_{2 d}$ symmetry. Such a defect in the neutral charge state would possess a partially occupied $e$ (doublet) level lying around mid-gap. This is a candidate for a JT distortion which lowers the symmetry, splitting the partially occupied $e$-level into two singlets. Cluster calculations [91] indicate there is a very large movement of one the radicals along the [011] direction during the relaxation, so that the structure of the neutral $\langle 100\rangle$ oriented split self-interstitial has $C_{1 h}$ symmetry. These calculations show that, in silicon, this has $0.31 \mathrm{eV}$ more energy than the $\langle 110\rangle$ form, whilst in Ge the difference in energies is greater at $0.51 \mathrm{eV}$.
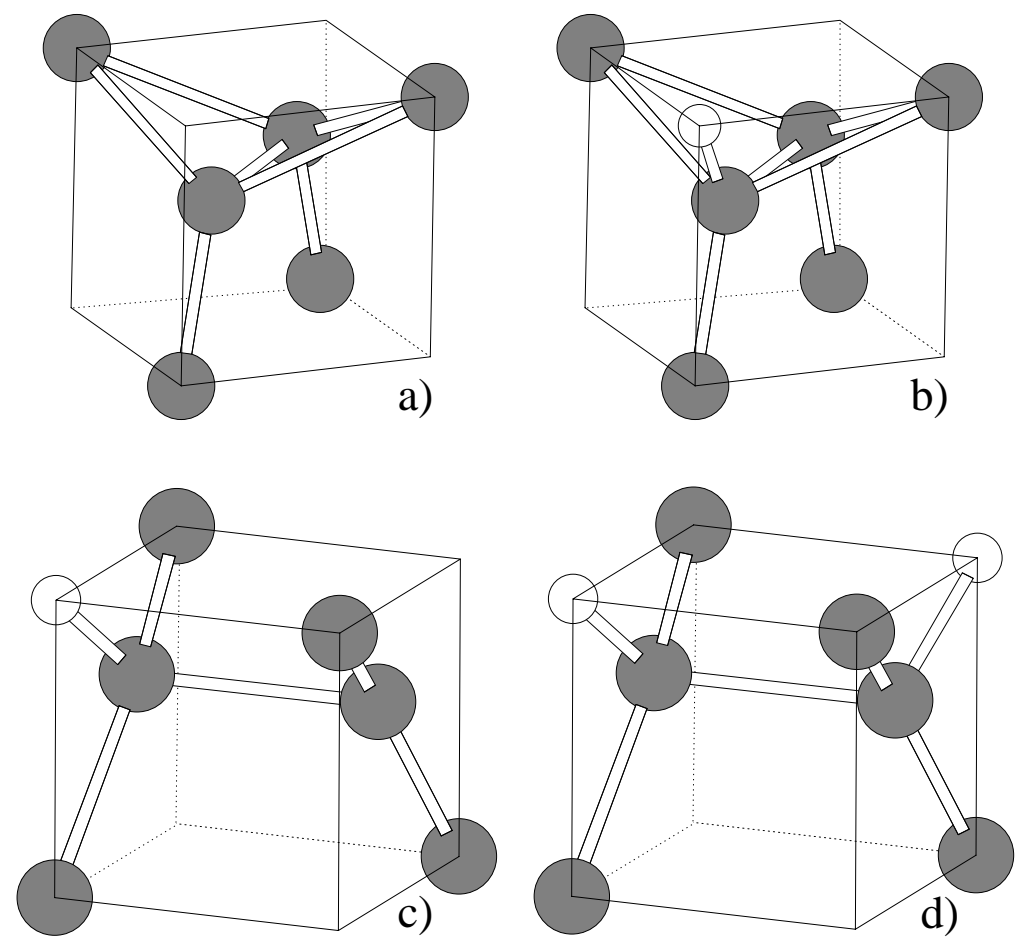

Fig. 5. Schematic figures of a) The $\langle 110\rangle$ split interstitial, b) The singly hydrogenated $\langle 110\rangle$ split interstitial, c) The singly hydrogenated $\langle 100\rangle$ split interstitial, d) $\mathrm{IH}_{2}$. 


\subsection{Interstitial complexes with one hydrogen atom}

We now consider the defect formed when one $\mathrm{H}$ atom is added to the split interstitial. Cluster calculations $[90,91]$ considered starting configurations consisting of $\langle 100\rangle\left(D_{2 d}\right)$ and $\langle 110\rangle$ $\left(C_{2 v}\right)$ self-interstitials with one of the two equivalent radicals hydrogenated, as shown in Fig. $5 \mathrm{c}$ and $5 \mathrm{~b}$ respectively. In each case the relaxed structure possesses $C_{1 h}$ symmetry. For both silicon and germanium, the difference in energies between the two configurations is small. The $\langle 100\rangle$ orientation possesses a lower energy by $0.24 \mathrm{eV}$ in $\mathrm{Si}$ over the $\langle 110\rangle$ derived structure, and in Ge the ground state is degenerate. These results for Si are in disagreement with supercell calculations [89] where a $\langle 110\rangle \mathrm{Si}-\mathrm{H}$ defect was found to be the ground state configuration.

These defects are paramagnetic in the neutral charge state and possess a deep singly occupied level. The calculated H-related LVMs along with their isotope shifts are given in Table 4. There have been no experimental reports of these modes. The stretch mode is considerably higher than the $1870 \mathrm{~cm}^{-1}$ found by the supercell calculation [89] for the $\langle 110\rangle$ configuration.

Table 4. Calculated frequencies $\left(\mathrm{cm}^{-1}\right)$ and characters of the IH defect.

\begin{tabular}{ccccccc}
\hline \hline & & \multicolumn{2}{c}{$\langle 100\rangle$} & \multicolumn{2}{c}{$\langle 110\rangle$} \\
& Symmetry & $\mathrm{H}$ & $\mathrm{D}$ & Symmetry & $\mathrm{H}$ & $\mathrm{D}$ \\
\hline \multirow{6}{*}{$\mathrm{Si}$} & $A$ & 2166.9 & 1556.3 & $A$ & 2190.8 & 1574.1 \\
& $B$ & 743.8 & 578.5 & $A$ & 882.0 & 649.7 \\
& $A$ & 724.2 & 578.8 & $B$ & 749.0 & 539.3 \\
& $A$ & 603.6 & 604.0 & $A$ & 630.8 & 610.1 \\
& $B$ & 577.2 & 547.4 & $A$ & 550.4 & 550.3 \\
\hline \multirow{6}{*}{$\mathrm{Ge}$} & $A$ & 2079.7 & 1478.9 & $A$ & 2073.7 & 1474.8 \\
& $A$ & 734.5 & 521.0 & $A$ & 800.5 & 567.3 \\
& $B$ & 729.9 & 517.8 & $B$ & 762.2 & 541.4 \\
\hline \hline
\end{tabular}

\subsection{Di-hydrogenated split interstitials}

The addition of two $\mathrm{H}$ atoms to the interstitial results in fully saturated bonds eliminating the requirement for a JT distortion. Cluster calculations show that for $\mathrm{Si}[32,89,91]$ and Ge [91] the $\langle 110\rangle$ defect spontaneously rotates into the one derived from the $\langle 100\rangle$ orientation, a schematic of which is shown in Fig. 5d. This possesses $C_{2}$ symmetry and the defect possesses a shallow doubly occupied level close to $E_{v}$. The polar and azimuthal angles were calculated to be $46^{\circ}$ and $10^{\circ}$ respectively for $\mathrm{Si}$, and $46^{\circ}(\mathrm{Ge})$ and $8^{\circ}$ for germanium. Here the polar angle is the 
angle between the $\mathrm{Si}-\mathrm{H}$ bond and $C_{2}$ axis along [001], and the azimuthal angle is that between the projection of the $\mathrm{Si}-\mathrm{H}$ bond onto the (001) plane and the $x$-axis.

The LVMs of the di-hydrogenated interstitial are given in Table 5. These modes are found to lie significantly below those of vacancy-hydrogen defects which were in turn about $7 \%$ higher than the experimental values [98]. This suggests that the Si and Ge radicals largely make weak $p$-like bonds with H. For the case of IHD, the presence of a single H mode lying midway between the two close-by $\mathrm{H}$ modes of $\mathrm{IH}_{2}$ implies that the $\mathrm{H}$ atoms are equivalent and weakly coupled. This is consistent with the calculations and with the view that the defects are interstitial and not vacancy like. Uniaxial stress studies [91] demonstrate that the defect possesses $C_{2}$ symmetry with polar and azimuthal angles of $48^{\circ} \pm 1^{\circ}$ and $0^{\circ} \pm 8^{\circ}$ respectively for $\mathrm{Si}$ whereas for Ge they are $45^{\circ} \pm 1^{\circ}$ and $3^{\circ} \pm 5^{\circ}$. The stretch modes are about $8 \%(\mathrm{Si})$ and $5 \%(\mathrm{Ge})$ too high and the $\mathrm{Si}$ ones are very similar to values found in smaller cluster calculations [90]. In both Si and Ge, the theory places the $A$ mode higher than the $B$ mode, in agreement with observation, with a small splitting of around $2-3 \mathrm{~cm}^{-1}$ in all cases. Four wag modes are predicted between $715-775 \mathrm{~cm}^{-1}$ (Si) and 690-790 $\mathrm{cm}^{-1}(\mathrm{Ge})$. However, in each case only two modes are observed. In Si, two wag modes are observed at 748 and $743 \mathrm{~cm}^{-1}$ having $B$ and $A$ symmetry respectively. In agreement with this, calculations find two modes at 774 and $768 \mathrm{~cm}^{-1}$ possessing $A$ and $B$ symmetry although the ordering is given incorrectly. Again, in Ge two wag modes are observed at 706 and $700 \mathrm{~cm}^{-1}$ but their intensities are too low to allow the determination of their symmetries. The calculations lead to two close-by modes at 787 and $785 \mathrm{~cm}^{-1}$ with $B$ and $A$ symmetries, in fair agreement with the experimental results.

\section{$7 \quad \mathrm{H}_{2}$ molecules}

Low temperature proton implantation gives no hint that molecular species can exist, but hydrogen molecules have long been suspected as a stable form of hydrogen in Si [99, 100]. Theory finds the energy of a $\mathrm{H}_{2}$ molecule at a $T$ site to lie about $0.2-0.4 \mathrm{eV}$ below $\mathrm{H}_{2}^{*}$. The dissociation energy of $\mathrm{H}_{2}$ into two $\mathrm{H}_{B C}$ was found to be $1.6 \mathrm{eV}$ [31] and the energy of two passivated $\mathrm{B}-\mathrm{H}$ complexes to be $0.5 \mathrm{eV}$ below that of the molecule. Consequently, at low temperatures a molecule should dissociate in the presence of boron acceptors. The first indications for the molecular species came from annealing studies which showed at $250^{\circ} \mathrm{C}$ hydrogen-boron complexes dissociate 
Table 5. Calculated frequencies $\left(\mathrm{cm}^{-1}\right)$ and characters of $\mathrm{IH}_{2}$ defects, compared with experiment [91].

\begin{tabular}{cccccc}
\hline \hline & Symmetry & $\mathrm{H}-\mathrm{H}$ & $\mathrm{H}-\mathrm{D}$ & $\mathrm{D}-\mathrm{D}$ & observed \\
\hline \multirow{4}{*}{$\mathrm{Si}$} & $A$ & 2144.7 & 2143.8 & 1540.2 & 1989.4 \\
& $B$ & 2142.9 & 1540.1 & 1539.9 & 1986.5 \\
& $A$ & 774.7 & 771.4 & 590.2 & 748.0 \\
& $B$ & 768.1 & 727.3 & 582.6 & 743.1 \\
& $B$ & 736.4 & 589.8 & 564.3 & \\
& $A$ & 717.5 & 579.8 & 555.0 & \\
$\mathrm{Ge}$ & $A$ & 2056.7 & 2055.1 & 1462.5 & 1883.5 \\
& $B$ & 2053.3 & 1461.3 & 1460.1 & 1881.8 \\
& $B$ & 787.4 & 784.9 & 558.8 & 705.5 \\
& $A$ & 784.7 & 555.9 & 555.7 & 700.3 \\
& $A$ & 725.0 & 712.4 & 514.7 & \\
\hline \hline
\end{tabular}

without any hydrogen effusing from the material [101]. The hydrogen produced by dissociation of such pairs is diamagnetic and gave no optical absorption lines. The suggestion was that it is present in molecular form although it was recognised that plasma damage leads to platelets which could trap this hydrogen. Subsequently NMR studies [102] on deuterated samples showed that molecules are present within voids in Si which had been plasma treated. However, the concentrations produced at that time appeared to be too small for the molecule to be detected by Raman scattering. Recently, Raman active modes around $4158 \mathrm{~cm}^{-1}$ were detected in plasma treated Si which had been etched to remove a surface layer of highly defective material produced by the plasma [103]. The claim was that these molecules were located at interstitial sites. This frequency is very close to the gas value and suggests that the surrounding lattice has little effect on the molecular frequency. This is unlike molecular hydrogen in GaAs where $\mathrm{H}_{2}$ modes red-shifted by $227 \mathrm{~cm}^{-1}$ have been detected by Raman scattering [104]. Furthermore, the intensity of the scattering due to the molecules in Si disappears above $\sim 400{ }^{\circ} \mathrm{C}$ along with a broad band of $\mathrm{Si}-\mathrm{H}$ vibrations around $2000 \mathrm{~cm}^{-1}$ attributed to platelets or voids [105, 106]. The present belief is that modes around $4158 \mathrm{~cm}^{-1}$ are due to molecules within void regions. Suggestions [107] that an EPR signal is due to hydrogen molecules have not been confirmed: the signal is now assigned to VH defects [71].

Nevertheless, circumstantial evidence for an interstitial form of molecule came from studies of $\mathrm{B}-\mathrm{H}$ defects in $\mathrm{Si}$ into which hydrogen had been introduced by high temperature in- 
diffusion [108]. SIMS and FTIR measurements on the B-H complexes in quenched samples showed most of the hydrogen was not bound to boron and yet there were no other $\mathrm{H}$ related defects detected optically at that time. Room temperature electron irradiation however produced $\mathrm{H}_{2}^{*}$ defects, supporting the idea that an invisible molecular fraction was present.

The first definitive evidence for an interstitial molecular species came from infra-red studies on thick crystals into which hydrogen had been in-diffused. $\mathrm{H}-\mathrm{H}$ stretch modes around $3700 \mathrm{~cm}^{-1}$ were detected. Subsequently, these modes were also detected in Raman scattering studies carried out on Si treated with a plasma at a low temperature (below $150^{\circ} \mathrm{C}$ ) where $\mathrm{Si}-\mathrm{H}$ modes characteristic of platelets were not detected [106]. In the FTIR study the trapping of the molecule by oxygen has played a crucial role which will now be discussed.

\subsection{Experimental background}

Early work identified an infra-red active mode at $1075.1 \mathrm{~cm}^{-1}$ in $\mathrm{Cz}-\mathrm{Si}$ into which hydrogen had been diffused at high temperature, which anomalously shifted upwards to $1076.3 \mathrm{~cm}^{-1}$ in deuterated samples $[109,110,111]$. This implies that hydrogen is part of the defect. Satellite lines due to the two naturally occurring isotopes of $\mathrm{Si}$ were shifted by about the same amount found for interstitial oxygen $[112,113]$. This suggests that oxygen is in its normal bond centred site and the hydrogen has perturbed its mode away from $1136 \mathrm{~cm}^{-1}$ characteristic of isolated interstitial oxygen. The defect was initially thought to be an $\mathrm{O}_{B C} \mathrm{H}_{i}$ pair but this was refuted by subsequent observations [112] of modes due to hydrogen dimers around $3700 \mathrm{~cm}^{-1}$. The intensities of these hydrogen-related modes were correlated with the oxygen modes. These later IR-absorption measurements revealed two forms of the $\mathrm{H}_{2}-\mathrm{O}_{B C}$ centre. The higher frequency hydrogen related modes labelled $\nu_{1}$ and $\nu_{2}$ associated with these defects are given in Table 6 . In the mixed H-D case, these modes are shifted roughly mid-way between the H-H and D-D combinations implying that they arise from a $\mathrm{H}-\mathrm{H}$ bond. The splitting of $\nu_{2}$ in the D-D case is not understood.

Upon heating to $110^{\circ} \mathrm{C}$, these bands almost disappear and the band labelled $\nu_{3}$ grows in intensity. This is a reversible process as $\nu_{1}$ and $\nu_{2}$ are reformed during cooling. This suggests that there is an equilibrium between molecules trapped near oxygen and those trapped at $T$ sites. This is supported by the observation that the number of the trapping sites is close to 
the number of possible $T$ sites in the lattice and that $\nu_{3}$ is detected in Float-zone silicon into which hydrogen has been diffused. It is not easy to give a convincing explanation of the effect of annealing unless interstitial molecules are involved — even though they are unexpectedly infrared active. The annealing studies give a binding energy of the molecule to oxygen to be $0.28 \pm 0.02 \mathrm{eV}$ and a barrier for molecular diffusion to be $0.78 \pm 0.05 \mathrm{eV}$ [111]. No DLTS lines have been correlated with these defects [110], consistent with the view that they are electrically inactive.

Table 6. Local vibrational modes $\left(\mathrm{cm}^{-1}\right)$ of hydrogen and oxygen related modes in silicon (after Pritchard et al. $[113,114])$.

\begin{tabular}{rccc}
\hline \hline & $\mathrm{H}_{2}$ & $\mathrm{HD}$ & $\mathrm{D}_{2}$ \\
\hline$\nu_{1}$ & 3788.9 & 3304.3 & 2775.4 \\
$\nu_{2}$ & 3730.8 & 3285.3 & $2715,2716.0$ \\
$\nu_{3}$ & 3618.3 & 3264.8 & 2642.5 \\
O-related mode & 1075.1 & 1076.3 & 1076.6 \\
\hline \hline
\end{tabular}

The molecular frequencies given here are very different from those around $4158 \mathrm{~cm}^{-1}$ detected in plasma treated material where platelets are known to form $[106,113,114,115,116$, 117, 103, 118, 105, 119, 120, 121, 122]. A Raman peak at $4158 \mathrm{~cm}^{-1}$, which has an associated deuterium-related peak at $2990 \mathrm{~cm}^{-1}$ and a rotational mode around $590 \mathrm{~cm}^{-1}$, is now believed to be related to hydrogen molecules in voids or platelets where the molecules are essentially in a gas phase [106]. These modes disappeared upon annealing at $\sim 450{ }^{\circ} \mathrm{C}$ along with IR active bands around $\sim 2100 \mathrm{~cm}^{-1}$ attributed to $\mathrm{Si}-\mathrm{H}$ bonds located on the inner surfaces of the voids. However, if the sample temperature was maintained below $150{ }^{\circ} \mathrm{C}$ during exposure to the plasma, neither the $\mathrm{Si}-\mathrm{H}$ void modes nor the molecular modes around $4200 \mathrm{~cm}^{-1}$ were detected. This suggests that the voids are not being formed. In these samples, however, Raman active modes at 3601 and $2622 \mathrm{~cm}^{-1}$ for hydrogen and deuterium have been detected at room temperature. These shifted upwards to the $\nu_{3}$ frequencies (Table 6) observed by Pritchard et al. for isolated $\mathrm{H}_{2}$ [114] at $10 \mathrm{~K}$. No $\mathrm{H}-\mathrm{D}$ modes were found, nor was a splitting of these modes due to ortho- and para- $\mathrm{H}_{2}$ molecules detected, in contrast to similar studies in GaAs [104]: this suggests that the molecule does not rotate in silicon, unlike $\mathrm{H}_{2}$ in GaAs.

The possibility that the $\nu_{i}$ modes are due to interstitial water molecules can be excluded. 
First, the $\mathrm{H}-\mathrm{D}$ mode lies almost midway between the $\mathrm{H}-\mathrm{H}$ and $\mathrm{D}-\mathrm{D}$ modes implying that a $\mathrm{H}-\mathrm{H}$ bond exists, and secondly the absence of an intense molecular wag mode at $\sim 1595 \mathrm{~cm}^{-1}$. Thus we conclude that $\mathrm{H}_{2}$ molecules exist in $\mathrm{Si}$ and are infrared active.

\subsection{Theoretical treatment of the hydrogen molecule in $\mathrm{Si}$}

Cluster and supercells have been employed in an attempt to ascertain the equilibrium site and vibrational properties of the hydrogen molecule in Si. The earliest calculations used semiempirical approaches. The earliest were performed were Corbett et al. [99] using the MNDO approach with a very small cluster $\left(\mathrm{Si}_{14} \mathrm{H}_{20}\right)$. The hydrogen molecule was found to be most stable at the $T$ site, aligned along [111]. The energy required to dissociate the molecule into two $\mathrm{H}^{0}$ atoms at $M$ sites (see Fig. 1) is calculated to be $1.6 \mathrm{eV}$. The barrier to molecular diffusion via the $H$ site (Fig. 1) is $2.7 \mathrm{eV}$. However, CNDO cluster based calculations by Mainwood and Stoneham $[100,123]$ found the molecule to be most stable at the $T$ site and aligned along [100]), with a rotation barrier of $0.093 \mathrm{eV}$ about the [110] axis. They also found the diffusion barrier to be $0.95 \mathrm{eV}$ and calculated the dissociation energies to two atoms at $T$ interstitial sites to be $\sim 4 \mathrm{eV}$.

In contrast, Deák et al. [22, 23, 124] using MINDO/3 cyclic cluster calculations on $\mathrm{Si}_{32}$ unit cells predicted that both $\mathrm{H}_{2}^{*}$ to be more stable than molecular hydrogen in the lattice by $0.49 \mathrm{eV}$. The molecule was found to be nearly degenerate in both the [100] and [111] alignments, with a $0.56 \mathrm{eV}$ barrier to diffusion.

More sophisticated, LDF calculations in supercells [16, 125] gave a [111] alignment although the barrier to rotation is negligible. The dissociation energy is $2.7 \mathrm{eV}$ into bond centred defects and the molecule is $0.4 \mathrm{eV}$ more stable than $\mathrm{H}_{2}^{*}$. Cluster calculations $[27,28]$ have also been used to examine the molecule. The molecular bond length was calculated to be $0.85 \AA$ at the $T$ site, with a vibrational frequency of $3561 \mathrm{~cm}^{-1}$. Importantly, in contrast with the supercell results, the most stable alignment of the molecule was found to be along [100] with a diffusion barrier of greater than $1 \mathrm{eV}$. The dissociation energy for conversion into bond centred defects was found to be $3.3 \mathrm{eV}$.

Estreicher et al. $[126,127]$ using both PRDDO (partial retention of differential diatomic overlap) in 14 and 44 atom clusters, and also HF calculations in 14 atom clusters, found the 
molecule at the $T$ site to have a bond length of $0.70 \AA$, and hence smaller than that of the free molecule, and a barrier to rotation of less than $0.1 \mathrm{eV}$. The preferred alignment was along [111] with the diffusion barrier less than $2 \mathrm{eV}$. In agreement with Deák et al. [22, 23], the molecule was found to be higher in energy than $\mathrm{H}_{2}^{*}$ by $0.82 \mathrm{eV}$ and $0.34 \mathrm{eV}$ respectively for the two methods.

A 32 atom supercell LDF calculation of Van de Walle et al. [15, 25, 31, 128, 129, 130] found, in agreement with the earlier cluster calculations [27, 28], that the molecule lies at the $T$ site along [100] although the energy of the [111] alignment is only $0.01 \mathrm{eV}$ greater. The $\mathrm{H}-\mathrm{H}$ bond length at $0.86 \AA$ exceeds that of the free molecule $(0.75 \AA)$ and leads to an anharmonic molecular frequency of $3396 \mathrm{~cm}^{-1}$, based on a polynomial fit of the molecular bond-length vs. energy [131]. These fit in well with the $\nu_{3}$ modes observed experimentally. The variations in the $\mathrm{H}-\mathrm{H}$ bond length in a variety of semiconductors [129] were accounted for by the size of the cavity surrounding the molecule leading to the greatest red-shift for the smallest cavity. This is explained by an increasing host charge density screening the binding between the hydrogen atoms. The diffusion and binding energies were found to be 1.1 and $2 \pm 0.5 \mathrm{eV}$ respectively and the molecule is $0.54 \mathrm{eV}$ lower in energy than $\mathrm{H}_{2}^{*}$. Recent LDF and GGA (generalised gradient approximation) calculations on molecules within $\mathrm{Si}_{32}$ and $\mathrm{Si}_{64}$ atom supercells $[132,133]$ found a preferred molecular alignment along [100], with a rotation barrier of about $0.003 \mathrm{eV}$. The diffusion barrier was found to be about $0.90 \mathrm{eV}$ and molecular frequencies of $3020 \mathrm{~cm}^{-1}$ for [100], and $3078 \mathrm{~cm}^{-1}$ for [111], alignments of the molecule. These frequencies became 3310 and $3363 \mathrm{~cm}^{-1}$ for the GGA calculations.

In contrast with these DFT results which imply a lower molecular frequency arising from the lengthened bond, Nakamura et al. [119, 134, 135, 136, 137] used both HF and DFT methods on small clusters $\left(\mathrm{Si}_{10} \mathrm{H}_{16}\right)$ and found that the molecule possesses a frequency very close to the gas value.

Recent cluster calculations [138] find the molecule to be stable when oriented either along [100], [111] or [110]. These three configurations are essentially degenerate in energy. The rotational barrier is also negligible, but the uncertainty in energy can easily exceed $0.1 \mathrm{eV}$, so the barrier is difficult to calculate accurately. This is similar to the situation in GaAs [139]. However, the para- and ortho-forms of the molecule are not detected in Si, which suggests that 
the barrier to rotation is larger in $\mathrm{Si}$ by $\sim 0.1 \mathrm{eV}[138]$. The calculated vibrational modes are given in Table 7 . These are all close together and lie within $90 \mathrm{~cm}^{-1}$ of the observed $\nu_{3}$ band. They are slightly lower than that found for the molecule trapped near oxygen $\left(3855.6 \mathrm{~cm}^{-1}\right)$. It is found that the $\mathrm{H}-\mathrm{H}$ length is $0.785 \AA$ for the [110] and [111] structures and $0.788 \AA$ for the [100] alignment. These are slightly longer than the gas value $(0.74 \AA)$.

Table 7. Calculated frequencies $\left(\mathrm{cm}^{-1}\right)$, of $\mathrm{H}_{2}$ molecules in Si with different alignments [138].

\begin{tabular}{cccc}
\hline \hline Alignment & $\mathrm{H}_{2}$ & $\mathrm{HD}$ & $\mathrm{D}_{2}$ \\
\hline$[110]$ & 3708.4 & 3217.1 & 2622.2 \\
{$[111]$} & 3713.0 & $3221.4,3221.6$ & 2625.5 \\
{$[100]$} & 3606.8 & 3128.6 & 2559.1 \\
\hline \hline
\end{tabular}

Molecules lying along [100] with $D_{2 d}$ symmetry would be infrared inactive, while those along [111] possess inequivalent $\mathrm{H}$ atoms. The latter defect produces two distinct HD modes as shown in Table 7 . As these are separated by $0.25 \mathrm{~cm}^{-1}$, they would give two bands with intensities corresponding to the different populations of the defects, contrary to observation. The $\nu_{3}$ band could not arise from these defects if the HD splitting was as large as this. The [110] orientation is infrared active with equivalent $\mathrm{H}$ atoms. Only one mode is then expected in the HD case. The $\nu_{3}$ band is therefore identified with a molecule lying along [110], although a [111] alignment cannot be completely ruled out. The stretch mode is about $90 \mathrm{~cm}^{-1}$ above that of the $\nu_{3}$ band but considerably lower than that calculated for an isolated molecule. Calculation [50] place this mode at $4424.8 \mathrm{~cm}^{-1}$, about $10 \%$ higher than the observed value, although anharmonicity must play a significant role in this overestimate.

The transition dipole moment of the molecule can be calculated directly. This is done by finding the change in the dipole moment of the cluster when the atoms are displaced according to their normal coordinates. The induced dipole lies along [001] (see Fig. 6) and arises as there is a slight displacement of the $\mathrm{H}$ atoms along this direction. The effective charge of the induced dipole is $0.1 e$ and comparable with that estimated for the molecule trapped by oxygen $[113,114]$. The molecular frequency is sensitive to the size of the cage surrounding it. If this is increased, the charge density at the centre of the cage would decrease, along with the $\mathrm{H}-\mathrm{H}$ bond length with a consequent increase in molecular frequency. This is seen in Fig. 7 where a relaxed molecule is placed in a cage of increasing size by scaling the surrounding $\mathrm{Si}-\mathrm{Si}$ 


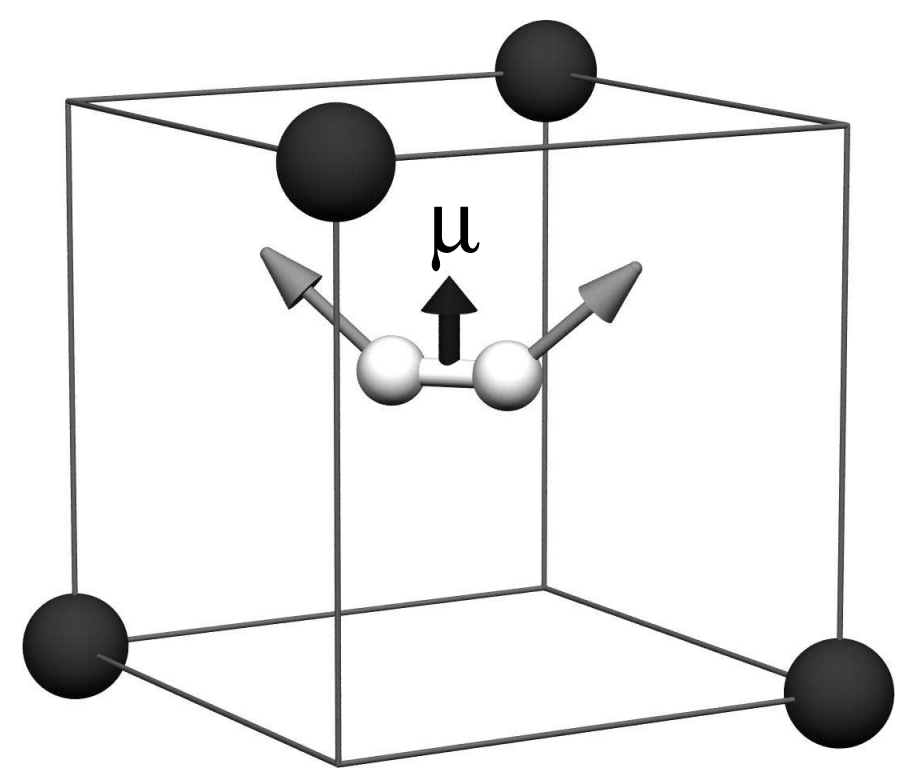

Fig. 6. Schematic figure of the stable form of the molecule at a $T$ site aligned along [110]. Arrows show an exaggerated movement of the atoms in the stretch mode leading to a transition dipole $\mu$ along [001].

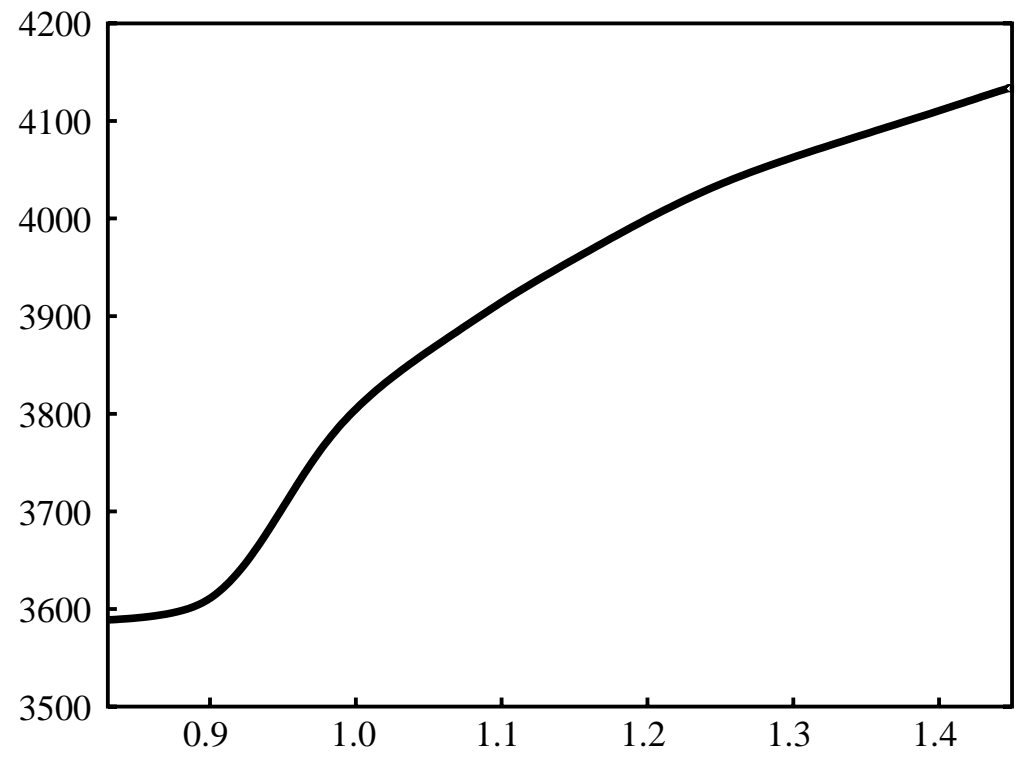

Fig. 7. Variation of molecular stretch frequency $\left(\mathrm{cm}^{-1}\right)$ verses multiples of the equilibrium interstitial cage size.

bonds. The gas value is reached for voids about 1.4 times the size of the actual cage, as shown in Fig. 7. This suggest that molecules within platelets might possess near gas frequencies [140].

A favoured model for the $\langle 111\rangle$ platelet (see Section 8), deduced from both transmission electron microscopy (TEM) studies [141] and theory [142], suggests that $\mathrm{H}$ forces apart the two nearby (111) planes by about $3 \AA$, and saturates the two surfaces of dangling bonds thus formed. This ideal structure does not involve any silicon vacancies. The vibrational modes of a 
$\mathrm{H}_{2}$ molecule trapped within a platelet have been calculated using LDF cluster methods [140]. The stretch frequencies for the molecule lie at $4385 \mathrm{~cm}^{-1}$ for $\mathrm{H}_{2}, 3796$ and $3804 \mathrm{~cm}^{-1}$ for HD, and $3101 \mathrm{~cm}^{-1}$ for $\mathrm{D}_{2}$. The modes of the hydrogen saturating the surrounding silicon atoms fall between 2117 and $2098 \mathrm{~cm}^{-1}$. The experimental values for platelets [143] lie at $\sim 2110$ and $\sim 1960 \mathrm{~cm}^{-1}$. The molecule has a bond length of $0.748 \AA$ when sited in the platelet, and is centred $2.459 \AA$ from the nearest passivating hydrogen atom and $3.255 \AA$ from the nearest structural silicon.

An alternative suggestion for the location of molecules is within a micro-void in the lattice $[144,145]$. Such voids are typically around $8 \mathrm{~nm}$ in diameter in crystalline silicon, this is somewhat large for current ab initio modelling techniques, but some insight into the behaviour of hydrogen in such defects can be obtained by simulation of smaller structures [138]. The smallest regular hydrogenated void that offers a more open environment than the crystal lattice is the passivated deca-vacancy, which is $1 \mathrm{~nm}$ across. The vibrational frequencies of the molecule in such a void lie at $4324.6 \mathrm{~cm}^{-1}$ for $\mathrm{H}_{2}, 3745.9$ and $3743.8 \mathrm{~cm}^{-1}$ for $\mathrm{HD}$, and 3057.9 for $\mathrm{D}_{2}$. The modes of the hydrogen atoms bound to silicon on the surface of the void fall into three bands at 2205-2191, 2141-2129 and 2108-2099 $\mathrm{cm}^{-1}$. Experimentally [144] bands at $\sim 2125, \sim 2087$ and $\sim 2073 \mathrm{~cm}^{-1}$ are observed. The molecular bond length is $0.759 \AA$ and the distance of the centre of the structure to a $\mathrm{H}$ atom on the void surface is $3.36 \AA$.

These results strongly suggest that Raman active modes at $4158 \mathrm{~cm}^{-1}$ found in H-plasma treated Si are due to molecules in open lattice defects where the electron density arising from the crystal is small and are not due to isolated molecules at interstitial sites. A realistic model of the void must have surface dangling bonds saturated by $\mathrm{H}$ [141] and a correlation of the Raman signals due to these $\mathrm{Si}-\mathrm{H}$ bonds and the $4158 \mathrm{~cm}^{-1}$ band is then to be expected, as has indeed been found by Leitch et al. [115, 116].

\subsection{Molecular diffusion barriers}

The molecule is not stable at the $H$ site but this site lies on a diffusion trajectory linking different $T$ sites. Cluster calculations [138] found that the energy of a molecule constrained to lie at the $H$ site is $0.72 \mathrm{eV}$ greater than that at a $T$ site and this then is an upper bound to the migration energy of the molecule. It is appreciably greater than the diffusion energy for 
$\mathrm{H}^{+}$or $\mathrm{H}^{0}$, which is around $0.43 \mathrm{eV}[46]$. The diffusion energy is in very good agreement with experimental value of $0.78 \mathrm{eV}[111]$

The curious discovery [146] that laser irradiation causes a decrease in the intensity of the $\nu_{3}$ band. This might to due to a photo-generated enhanced movement of the molecule at cryogenic temperatures. Such an effect might arise if the laser generated electron-holes pairs, which then destabilised the molecule. Alternatively, the molecule might dissociate as a result of a resonant absorption of vibrational energy. Cluster calculations [140] show that the diffusion barrier drops from $0.73 \mathrm{eV}$ in the neutral case, to 0.33 and $0.46 \mathrm{eV}$ for clusters charged to +2 or -2 respectively, with an expansion of the bond length whilst the molecule is at the hexagonal site from

$0.788 \AA$ to 0.901 and $0.930 \AA$ respectively. The bond length at the tetrahedral site is insensitive to charge state. However, this drop in diffusion barrier is insufficient to account for motion at cryogenic temperatures and the effect remains unexplained.

\subsection{Complexes of oxygen with hydrogen molecules}

We have already described the evidence that the molecule can be trapped near impurities such as oxygen, taking advantage of the opening of a lattice cage caused by this impurity. We now describe the results of cluster calculations on these defects [147]. There are several obvious locations for the molecule close to $\mathrm{O}_{i}$ in the silicon lattice but since the binding between the molecule and the $\mathrm{O}$ atom is so weak, these are close in energy and difficult to distinguish. Furthermore, it seems that there are a large number of configurations which differ only in the orientation of the molecule and all of these have to be considered as candidate structures.

Suppose that the molecule resides near a $T_{d}$ cage site and oxygen decorates one of the nearby $\mathrm{Si}-\mathrm{Si}$ bonds and consider the case when the $\mathrm{O}$ atom bridges a [11̄] $\mathrm{Si}-\mathrm{Si}$ bond forming part of the cage surrounding the $T$ site as shown in Fig. 8. The molecule in the relaxed configuration then is oriented almost perpendicular to the $\mathrm{Si}-\mathrm{O}-\mathrm{Si}$ bond and nearly along [110]. The $\mathrm{H}-\mathrm{H}$ bond length is $0.77 \AA$ and each $\mathrm{H}$ atom is about $2.37 \AA$ from O. It has been pushed slightly away from the $T$ site as the nearest two Si atoms are $2.19 \AA$ but these are not bonded to O. The calculated vibrational modes [147] are given in Table 8.

The $\mathrm{H}_{2}$ stretch mode at $3855 \mathrm{~cm}^{-1}$ is again lower than that calculated for an isolated molecule. The molecular frequency depends on the size of the surrounding cage. The $3855 \mathrm{~cm}^{-1}$ 


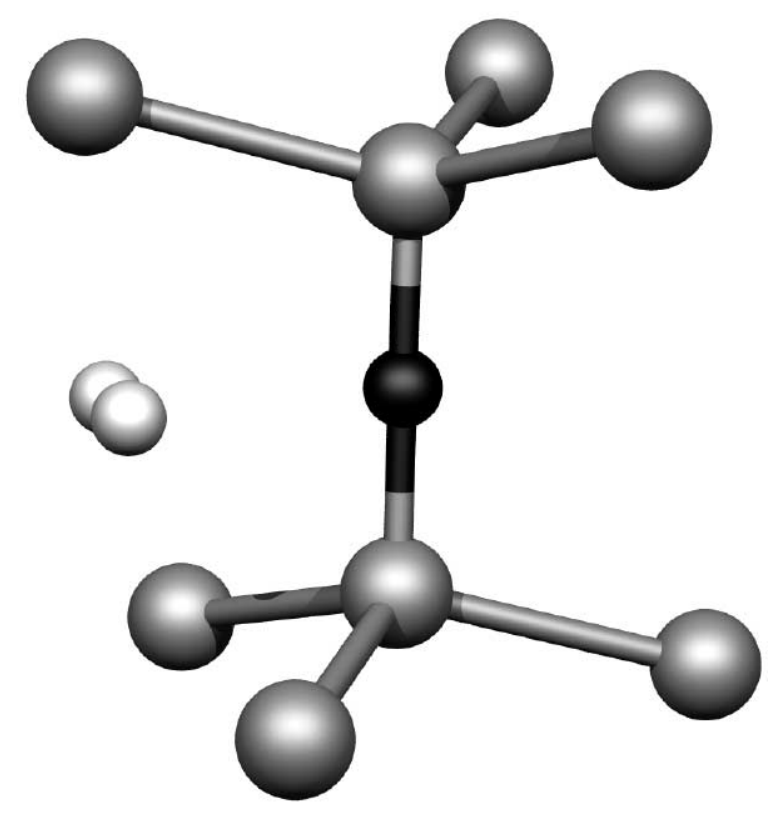

Fig. 8. Schematic illustration of $\mathrm{O}_{i}+\mathrm{H}_{2}$ defect.

Table 8. Local vibrational modes $\left(\mathrm{cm}^{-1}\right)$ of the hydrogen-molecule- $\mathrm{O}_{B C}$ complex in silicon, as illustrated in Fig. 8. Experimental frequencies are listed in Table 6.

\begin{tabular}{ccccc}
\hline \hline $\mathrm{H}_{2}{ }^{16} \mathrm{O}_{B C}$ & $\mathrm{HD}^{16} \mathrm{O}_{B C}$ & $\mathrm{DH}^{16} \mathrm{O}_{B C}$ & $\mathrm{D}_{2}{ }^{16} \mathrm{O}_{B C}$ & $\mathrm{H}_{2}{ }^{18} \mathrm{O}_{B C}$ \\
\hline 3855.6 & 3339.5 & 3350.1 & 2726.7 & 3855.6 \\
1129.8 & 1129.7 & 1129.6 & 1129.5 & 1078.1 \\
733.3 & 685.6 & 690.6 & 678.6 & 731.4 \\
696.2 & 663.2 & 654.6 & 653.7 & 685.3 \\
655.3 & 650.1 & 645.0 & 630.9 & 649.0 \\
643.6 & 627.6 & 627.4 & 536.3 & 638.9 \\
630.6 & 539.5 & 542.6 & 533.6 & 622.6 \\
576.7 & 536.0 & 536.1 & 532.8 & 576.1 \\
\hline \hline
\end{tabular}

mode lies close to the experimental modes around $3750 \mathrm{~cm}^{-1}$ associated with oxygen. The two $\mathrm{H}$ atoms are almost equivalent and this explains why only one $\mathrm{H}-\mathrm{D}$ mode is present although the calculations show a $10 \mathrm{~cm}^{-1}$ splitting caused by deviations, due to numerical noise, from ideal $C_{1 h}$ symmetry.

The mode at $1129.8 \mathrm{~cm}^{-1}$ is clearly due to $\mathrm{O}_{i}$ as it shifts downwards by only $0.3 \mathrm{~cm}^{-1}$ when D replaces $\mathrm{H}$ but by $52 \mathrm{~cm}^{-1}$ with ${ }^{18} \mathrm{O}$. This is comparable with the $51 \mathrm{~cm}^{-1}$ shift observed in the $1136 \mathrm{~cm}^{-1}$ mode of $\mathrm{O}_{i}$ when ${ }^{18} \mathrm{O}$ replaces ${ }^{16} \mathrm{O}$. The small downward shifts on replacement of $\mathrm{H}$ with $\mathrm{D}$ demonstrate that there is very little direct coupling between $\mathrm{O}_{i}$ and the molecule and this is essential to these arguments, based on anharmonicity, if an upward shift is to be seen in the deuterated case. It is not clear why the experimental O mode is shifted downwards 
by as much as $65 \mathrm{~cm}^{-1}$ from that of isolated $\mathrm{O}_{i}$.

The modes at 733 to $576 \mathrm{~cm}^{-1}$ all involve the movement of $\mathrm{H}$ and have not been detected so far. They represent $\mathrm{H}_{2}$ bend and librational modes. Their shifts with ${ }^{18} \mathrm{O}$ given in Table 8 demonstrate that many of them involve the movement of $\mathrm{O}$.

Of particular interest here is the mode at $577 \mathrm{~cm}^{-1}$. This represents a librational mode as the two $\mathrm{H}$ atoms are displaced almost parallel to their bond. It can be argued that the anomalous frequency shift of the $1075 \mathrm{~cm}^{-1}$ band is to be understood through an anharmonic coupling between an overtone, or combination band, of these low frequency modes and the $\mathrm{O}$ mode resulting in a Fermi resonance. Let $\left|n_{\mathrm{Ox}}\right\rangle$ be the $n^{\text {th }}$ oscillator state for the vibrations of the oxygen atom whose fundamental occurs at $\nu_{\mathrm{O}}=1129.8 \mathrm{~cm}^{-1}$, and $\left|m_{\mathrm{H}}\right\rangle$ be the $m^{\text {th }}$ state for a mode whose frequency, $\nu_{\mathrm{H}}$, is about half that of the $\mathrm{O}_{i}$ mode, i.e. either the modes in the region of $550 \mathrm{~cm}^{-1}$. The states of the coupled system are then described by linear combinations of $\left|n_{\mathrm{Ox}}, m_{\mathrm{H}}\right\rangle$. The effect of anharmonicity, $V$, is to couple together these states and second order perturbation theory gives the shift in the energy of the $\left|1_{\mathrm{Ox}}, 0_{\mathrm{H}}\right\rangle$ state to be dominated by

$$
-\frac{\left|\left\langle 1_{\mathrm{Ox}}, 0_{\mathrm{H}}|V| 0_{\mathrm{Ox}}, 2_{\mathrm{H}}\right\rangle\right|^{2}}{\left(\nu_{\mathrm{O}}-2 \nu_{\mathrm{H}}\right)} \text {. }
$$

This follows as the energy denominator is particularly small for these modes. The perturbation is negative for the $577 \mathrm{~cm}^{-1}$ mode and lowers the energy of the $\left|1_{\mathrm{Ox}}, 0_{\mathrm{H}}\right\rangle$ state, and hence that of the fundamental transition. On the other hand, when $\mathrm{H}$ is replaced by $\mathrm{D}$, as the frequency of this mode drops below $575 \mathrm{~cm}^{-1}$, the perturbation acts to raises the energy of the state. Thus the two cases reinforce the tendency to depress the $\mathrm{O}$ mode in the $\mathrm{H}$ case below that of $\mathrm{D}$. Another ways of describing the effect is an anti-crossing between the $\mathrm{O}$ mode and an overtone of the librational mode (as shown in Fig. 9). If this mechanism is correct, there have to be unreported modes in the $550 \mathrm{~cm}^{-1}$ region.

Other positions for the molecule have also been investigated. A second, almost degenerate configuration occurs when the molecule lies in the mirror plane containing the $\mathrm{O}_{i}$. This has similar modes to the first and could account for the second defect which is observed. A third possibility is that the molecule lies along the $\mathrm{Si}-\mathrm{O}-\mathrm{Si}$ axis near a $T$ site. This site is stable but the molecule is then close to the $\mathrm{Si}$ neighbour of $\mathrm{O}_{i}$ and this results in an $\mathrm{O}$ related mode that is strongly coupled with $\mathrm{H}$ in conflict with the experiment. However, the energy of this structure 


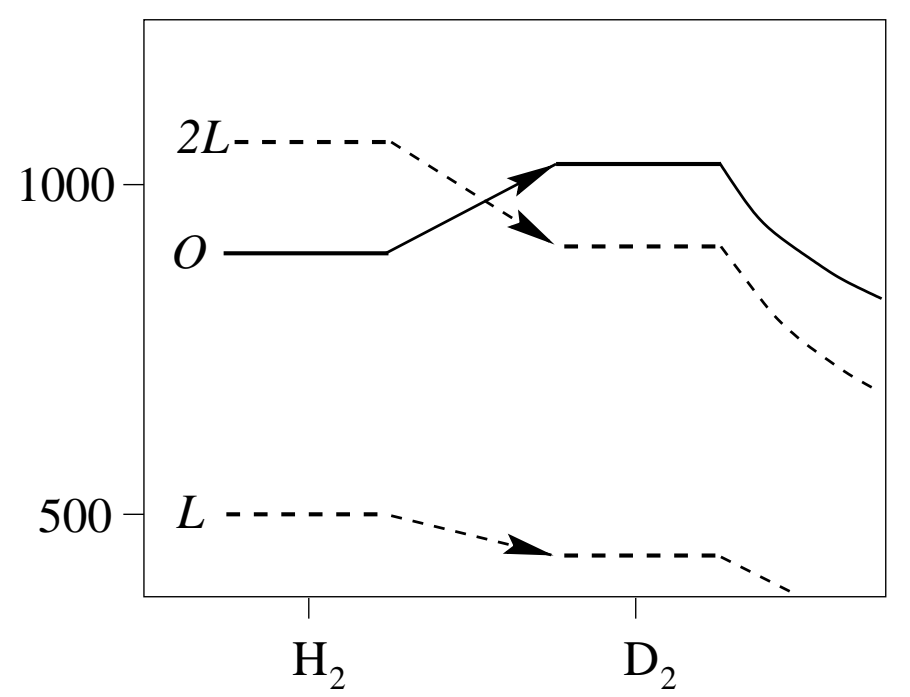

Fig. 9. Schematic of the anti-crossing of the oxygen mode and an overtone of a low-lying librational mode.

appears to be lower than that shown in Fig. 8 by $0.5 \mathrm{eV}$. This may be a due to the proximity of $\mathrm{O}_{i}$ to the surface of the cluster and further investigations are needed to clarify the most stable configuration.

\subsection{Other O-H complexes in silicon}

Several modes due to $\mathrm{O}-\mathrm{H}$ defects have been detected in proton-implanted $\mathrm{Cz}-\mathrm{Si}$ [148]. An oxygen-hydrogen complex $\mathrm{OH}_{I}$ was detected by FTIR in Si proton implanted around $\sim 20 \mathrm{~K}$ which, on annealing at $\sim 200 \mathrm{~K}$, converted to a second complex $\mathrm{OH}_{I I}$. Oxygen and deuterium isotopic studies confirmed the presence of one oxygen and one hydrogen atom in each defect. $\mathrm{OH}_{I}$ has an oxygen related IR active mode at $1077 \mathrm{~cm}^{-1}$ and a hydrogen mode at $1879 \mathrm{~cm}^{-1}$, whilst $\mathrm{OH}_{I I}$ has an oxygen related mode at $1028 \mathrm{~cm}^{-1}$ and a hydrogen mode at $1830 \mathrm{~cm}^{-1}$ (see Table 9 for the measured frequencies). On implantation with $\mathrm{H}$ and $\mathrm{D}$, both the $\mathrm{OH}$ complexes show only two hydrogen related lines, implying that a single hydrogen atom is present in each type of defect. The complexes were tentatively assigned to two forms of $\mathrm{O}_{B C} \mathrm{H}_{B C}^{+}$defects.

First principles calculations [148] were performed on three candidate structures for the $\mathrm{OH}$ defects. The hydrogen atom in the complex was placed at one of three possible different bond centred sites near the bond-centred oxygen, i.e. the nearest, next-nearest and third-nearest bond centres. It was suggested, based on the isotopic shifts of these structures that $\mathrm{OH}_{I I}$ might be due to $\mathrm{H}_{B C}$ at the second or third nearest neighbour sites, and $\mathrm{OH}_{I}$ might be due to $\mathrm{H}_{B C}$ at the third or fourth nearest neighbour sites. 
Table 9. Local vibrational modes $\left(\mathrm{cm}^{-1}\right)$ for the $\mathrm{OH}_{I}$ and $\mathrm{OH}_{I I}$ models [148].

\begin{tabular}{ccccc}
\hline \hline \multirow{2}{*}{ Isotope mix } & \multicolumn{2}{c}{$\mathrm{OH}_{I}$} & \multicolumn{2}{c}{$\mathrm{OH}_{I I}$} \\
& O-mode & H-mode & O-mode & H-mode \\
\hline${ }^{16} \mathrm{OH}$ & 1076.9 & 1879.2 & 1028.5 & 1830.4 \\
${ }^{16} \mathrm{OD}$ & 1076.7 & 1358.0 & 1032.3 & 1329.1 \\
${ }^{18} \mathrm{OH}$ & 1028.3 & 1879.1 & 987.1 & 1830.1 \\
${ }^{18} \mathrm{OD}$ & 1028. & 1358.0 & 987.7 & 1330.6 \\
\hline \hline
\end{tabular}

As with the $\mathrm{O}_{i} \mathrm{H}_{2}$ complexes observed by Markevitch et al. and Pritchard et al. (see Section 7.1), the oxygen related line of the $\mathrm{OH}_{I I}$ complex shifts upwards on replacing hydrogen with deuterium, presumably due to a similar anharmonic effect to that outlined in Section 7.4.

Diffusion of $\mathrm{H}$ into oxygen rich $\mathrm{Si}$ also leads to enhanced $\mathrm{O}$ diffusion in a temperature region around $350^{\circ} \mathrm{C}$, with an activation energy of 1.7-2.0 eV (Newman et al. [149], Stein and Hahn [150]). No stable O-H complexes have been reported in the temperature range at which enhanced diffusion occurs, and it is thought that $\mathrm{H}$ catalyses the movement of $\mathrm{O}$. There are several theories for the enhancement in the reorientation rate of $\mathrm{O}_{i}$ in the presence of $\mathrm{H}$. During the movement of $\mathrm{O}$ from one $B C$ site to the next, it must pass close to the $C$ site and is then necessarily bonded to three Si atoms, with one of the Si atoms having a dangling bond. This is the saddle point for normal O diffusion. Cluster calculations of the energy difference from the starting configuration determines the diffusion energy of $2.8 \mathrm{eV}$. However, its energy can be lowered if the Si atom with the dangling bond is able to make a strong bond with a $\mathrm{H}$ atom that happens to be in close vicinity. The lowest energy for $\mathrm{H}$ near $\mathrm{O}$ is at an $A B$ site opposite to $\mathrm{Si}-\mathrm{O}-\mathrm{Si}$. Relative to one of these two sites, the energies of $\mathrm{H}_{B C}$ and the three $\mathrm{H}_{T_{d}}$ sites are $1.5 \mathrm{eV}$ and $0.75 \mathrm{eV}$ respectively. With $\mathrm{H}$ at the $A B$ site, the energy difference between $\mathrm{O}$ at a $B C$ and at a $C$ site is only $0.2 \mathrm{eV}$. Thus the barrier of $2.8 \mathrm{eV}$ for normal O-diffusion has been effectively eliminated by the presence of $\mathrm{Si}-\mathrm{H}$. Now, when $\mathrm{O}$ moves away from the C-site, the energy increases because the $\mathrm{Si}-\mathrm{H}$ bond starts to weaken and a normal $\mathrm{Si}-\mathrm{Si}$ bond starts to form. The new activation barrier is calculated to be $1.4 \mathrm{eV}$ : somewhat lower than the observed barrier. When the $\mathrm{O}$ atom moves to its new bond centred site $\mathrm{H}$ is no longer in the lowest energy configuration and must jump to a new anti-bonding site. It has to move to the further of these sites if long ranged $\mathrm{O}$ migration is to occur. 
In contradiction with this, Estreicher [151] finds that $\mathrm{H}_{B C}-\mathrm{Si}-\mathrm{O}_{B C}$ is more stable than $\mathrm{H}_{A B^{-}}$ $\mathrm{Si}-\mathrm{O}_{B C}$. The energy for a metastable configuration $\mathrm{H}_{T_{d}}-\mathrm{Si}-\mathrm{O}_{B C}$ was $0.66 \mathrm{eV}$ higher. He then argued that when $\mathrm{H}$ is located near to this metastable $\mathrm{T}_{d}$ site, it is able to lower the barrier for $\mathrm{O}$ migrating to the $C$ site through the formation of the $\mathrm{Si}-\mathrm{H}$ bond and leaving $\mathrm{O}$ bonded to two atoms. This barrier, when $\mathrm{H}$ is initially at the $T_{d}$ site, is $1.25 \mathrm{eV}$ and is much lower than in the absence of $\mathrm{H}$. The final configuration consists of $\mathrm{H}$ and $\mathrm{O}$ at adjacent $B C$ centres. This is not the starting point of the process and it is necessary to assume that $\mathrm{H}$ can leave the $B C$ site after the O hop.

Recently, first principles molecular dynamics has been used to determine the migration barrier for oxygen in the presence of hydrogen [18]. The authors find that in $p$-type $\mathrm{Si}, \mathrm{H}^{+}$lies at a bond centred site adjacent to the oxygen atom and migrates together with $\mathrm{O}$ so that at the saddle point, $\mathrm{H}$ saturates a $\mathrm{Si}$ dangling bond. The activation energy of oxygen is reduced from $2 \mathrm{eV}$ to about $1.5 \mathrm{eV}$ in the presence of hydrogen. The problem with this study is that the starting structure, where $\mathrm{O}$ and $\mathrm{H}$ share a common $\mathrm{Si}$ atom, seems to be at variance with the experimental results of Bech Nielsen et al. [148] which show that there is a very weak interaction between $\mathrm{O}$ and $\mathrm{H}$.

Further work is necessary to determine the precise mechanism.

\section{Evolution of $\mathrm{H}$ species in $\mathrm{Si}$}

The types of hydrogen defect present in silicon depend critically on the manner in which the impurity is introduced, the impurities and defects present in $\mathrm{Si}$, and the subsequent treatment of the sample. Common techniques used to introduce hydrogen are, as we have seen, i) proton implantation, ii) in-diffusion at high temperatures of $\sim 1200^{\circ} \mathrm{C}$ from the molecular species followed by a quench, iii) via a plasma, and iv) by room temperature wet etching.

\subsection{Proton Implantation}

At low temperatures, protons with energy greater than $150 \mathrm{eV}$ generate $\mathrm{V}^{-}, \mathrm{V}^{2-}, \mathrm{V}^{0}, \mathrm{H}^{+}$and

I. The number of vacancies per proton depends on the beam energy. Fabian et al. [152] suggest that $7.5 \mathrm{keV}$ protons produce on average 3.5 vacancies per ion, but $0.5 \mathrm{keV}$ protons only produce 0.5 vacancies per ion at the end of the range of the proton. FTIR studies combined with DLTS 
show that the dominant $\mathrm{H}$ species at the lowest of temperatures is $\mathrm{H}_{B C}^{+}[34]$. This must be charge compensated by another defect which is probably $\mathrm{V}^{-}$or $\mathrm{V}^{2-}$.

The equilibrium concentrations of $\mathrm{H}^{+}, \mathrm{H}^{-}$and neutral $\mathrm{H}\left(\mathrm{H}^{0}\right)$ can be written:

$$
\begin{aligned}
& {\left[\mathrm{H}^{+}\right]=\frac{[\mathrm{H}]}{Z} \exp \left\{-E\left(\mathrm{H}^{+}\right) / k T\right\},} \\
& {\left[\mathrm{H}^{0}\right]=\frac{[\mathrm{H}]}{Z} \exp \left\{-\left[E\left(\mathrm{H}^{0}\right)-E_{F}\right] / k T\right\},} \\
& {\left[\mathrm{H}^{-}\right]=\frac{[\mathrm{H}]}{Z} \exp \left\{-\left[E\left(\mathrm{H}^{-}\right)-2 E_{F}\right] / k T\right\} .}
\end{aligned}
$$

Here $[\mathrm{H}]$ is the total concentration of hydrogen and $E\left(\mathrm{H}^{q}\right)$ is the energy of a species with charge q. Thus we can write:

$$
\left[\mathrm{H}^{0}\right]=\left[\mathrm{H}^{+}\right] \exp \left\{-\left(E[0 /+)-E_{F}\right] / k T\right\} \quad \text { and } \quad\left[\mathrm{H}^{-}\right]=\left[\mathrm{H}^{0}\right] \exp \left\{-\left[E(-/ 0)-E_{F}\right] / k T\right\} \text {. }
$$

We take $E(0 /+)$ to lie at $E_{c}-0.16 \mathrm{eV}$ and $E(-/ 0)$ to be $E_{c}+\Delta-0.56 \mathrm{eV}$ where $E_{c}-0.56 \mathrm{eV}$ is the $(-/ 0)$ level deduced from studies of $\mathrm{Mu}^{-}[49] . \Delta$ is the energy difference between a neutral $\mathrm{H}$ atom at this site and the stable $B C$ site. We suppose $\Delta \sim 0.1-0.2 \mathrm{eV}$ and the effective $(-/ 0)$ level for $\mathrm{H}$ is then around $E_{c}-0.4 \mathrm{eV}$. This is within $0.2 \mathrm{eV}$ of the acceptor level deduced by capacitance studies on $n$-type $\mathrm{Si}[29]$ as well as the calculations described in Section 9.

Clearly, the ratio of $\mathrm{H}^{-}$to $\mathrm{H}^{+}$is then given by

$$
\left[\mathrm{H}^{-}\right]=\left[\mathrm{H}^{+}\right] \exp \left\{-\left[E(0 /+)+E(-/ 0)-2 E_{F}\right] / k T\right\}
$$

Thus $\mathrm{H}^{-}$is not present at low temperatures if $E_{F}$ is lower than $(E(0 /+)+E(-/ 0)) / 2 \sim$ $E_{c}-0.3 \mathrm{eV}$. However, increasing the numbers of electrons in the system, as in photo-generation (we suppose that these are attracted to $\mathrm{H}^{+}$whereas holes are repelled) or injecting electrons under forward bias, will elevate the Fermi-level and increase the concentration of $\mathrm{H}^{-}$[38]. For this process, $\mathrm{H}^{+}$must capture an electron becoming $\mathrm{H}^{0}$ leading to the $A A 9 \mathrm{EPR}$ spectrum. This, however, is only a metastable defect and $\mathrm{H}^{0}$ will attempt to hop to the $T$ site, pick up another electron and become $\mathrm{H}^{-}$. However, these processes are in competition with a loss of $\mathrm{H}^{+}$caused by diffusion of say $\mathrm{V}^{2-}$ to $\mathrm{H}^{+}$leading to the creation of $\mathrm{VH}^{-}$.

In addition, if $\mathrm{H}^{0}$ can jump or tunnel into the $T$ site leading to $\mathrm{H}^{-}$, then we must expect that another $\mathrm{H}^{+}$will be able to combine with this $\mathrm{H}^{-}$to create $\mathrm{H}_{2}$ or $\mathrm{H}_{2}^{*}$. Reorientation of $\mathrm{H}^{0}$ is known to be activated with a barrier of $0.43 \mathrm{eV}[46]$ around $200 \mathrm{~K}$ and motion of $\mathrm{H}^{0}$ 
would appear to be frozen out at lower temperatures unless tunnelling takes place. There has been recent suggestions based on channelling data that tunnelling of $\mathrm{H}^{+}$can take place at low temperatures $<200 \mathrm{~K}[152]$. Here, the diffusivity is found to be proportional to $T^{n}$ with $n=5.6$. Thus it seems possible to imagine the formation of $\mathrm{H}_{2}^{*}$ without the necessity of a vacancy or interstitial.

On the other hand $\mathrm{V}^{2-}$ is known to diffuse around $80 \mathrm{~K}$ and can complex with $\mathrm{H}^{+}$, forming $\mathrm{VH}^{-}$. The $20 \%$ drop in the intensity of the vibrational band at $1998 \mathrm{~cm}^{-1}$ band assigned to $\mathrm{H}^{+}$ around $80 \mathrm{~K}$ is attributed to this reaction [34]. However, the main annealing sequence for this band occurs around $200 \mathrm{~K}$ and this involves the activated movement of $\mathrm{H}^{+}$. In this temperature range $\mathrm{V}_{2}$ defects are formed. The consequent raising of $E_{F}$ must force the formation of more $\mathrm{H}^{-}$and thus of $\mathrm{H}_{2}^{*}$.

Alternatively, diffusing vacancies can complex with $\mathrm{H}^{-}$forming $\mathrm{VH}$ and $\mathrm{VH}_{2}$ defects. It has been suggested that these complex with interstitials yielding $\mathrm{H}_{2}^{*}$ [153], although it is not clear that $\mathrm{IH}_{2}$ defects are also formed in this temperature range. Which of these many processes leading to $\mathrm{H}_{2}^{*}$ is dominant is not known although it does appear that $\mathrm{H}_{2}^{*}$ is created before $\mathrm{VH}_{2}$ or $\mathrm{IH}_{2}$ defects [34].

If one regards $\mathrm{H}_{2}^{*}$ as a defect with two nearby $\mathrm{Si}-\mathrm{H}$ bonds, then conversion of this into the more stable $\mathrm{H}_{2}$ molecule would require an activation energy of around $2 \mathrm{eV}$. Thus $\mathrm{H}_{2}^{*}$ would be expected to be stable up to about $180^{\circ} \mathrm{C}$ which is consistent with annealing studies.

Annealing proton implanted Si around $200{ }^{\circ} \mathrm{C}$ leads to the formation of platelets [154], while Rutherford backscattering experiments have been interpreted [155] as showing that between 150 and $350^{\circ} \mathrm{C}$, molecules are produced.

\subsection{High temperature in-diffusion}

We now consider the case of $\mathrm{H}_{2}$ introduced from the gas phase. This is usually carried out by heating the sample in a quartz ampoule containing hydrogen gas at temperatures around $1100{ }^{\circ} \mathrm{C}$. At these temperatures there is a copious supply of $e$ and $h, \mathrm{H}^{+}$, and $\mathrm{H}^{-}$. The solubility of hydrogen in Si is observed to be proportional to the square root of the gas pressure $p$ [47], implying that molecular hydrogen within $\mathrm{Si}$ is dissociated at these temperatures. If there is equilibrium between $\mathrm{H}$ inside $\mathrm{Si}$ and $\mathrm{H}_{2}$ outside, then the chemical potential $\mu(\mathrm{H})$ for $\mathrm{H}$ inside 
the crystal is related to the chemical potential of $\mathrm{H}_{2}$ outside by:

$$
\mu(\mathrm{H})=\mu\left(\mathrm{H}_{2}\right) / 2
$$

Now, from the statistical mechanics of an ideal gas,

$$
\begin{aligned}
\mu\left(\mathrm{H}_{2}\right) & =E_{g}\left(\mathrm{H}_{2}\right)+k T \ln \left\{\left(\frac{p}{k T}\right)\left(\frac{2 \pi \hbar^{2}}{m k T}\right)^{3 / 2} \frac{1}{Z_{\mathrm{rot}}}\right\}, \\
& =E_{g}\left(\mathrm{H}_{2}\right)+k T \ln [D(g)]
\end{aligned}
$$

Here $E_{g}\left(\mathrm{H}_{2}\right)$ is the free energy of a gas molecule, including vibrational modes and $Z_{\text {rot }}$ is the rotational partition function. At these temperatures, $Z_{\text {rot }} \sim 17$. Thus the concentrations of $\mathrm{H}$ and $\mathrm{H}_{2}$ inside the crystal are given by:

$$
\begin{aligned}
{[\mathrm{H}] } & =2 N \exp \{-[E(\mathrm{H})-\mu(\mathrm{H})] / k T\}, \\
& =2 N \sqrt{D(g)} \exp \left\{-\left[E(\mathrm{H})-E_{g}\left(\mathrm{H}_{2}\right) / 2\right] / k T\right\}, \\
{\left[\mathrm{H}_{2}\right] } & =N D(g) \exp \left\{-\left[E\left(\mathrm{H}_{2}\right)-E_{g}\left(\mathrm{H}_{2}\right)\right] / k T\right\} .
\end{aligned}
$$

Here $N$ is the density of $T$ sites which is half the density of $B C$ sites and $E$ is the free energy of the species inside the crystal. The equilibrium molecular concentration inside the crystal is related to the density of the monatomic species by:

$$
\left[\mathrm{H}_{2}\right]=\frac{[\mathrm{H}]^{2}}{4 N} \exp \left\{-\left[E\left(\mathrm{H}_{2}\right)-2 E(\mathrm{H})\right] / k T\right\} \text {. }
$$

The quantity $D$ evaluates to $6 \times 10^{-8}$ assuming the ampoule originally contained hydrogen gas at STP. The first principles calculations give the energy of the $\mathrm{H}$ atom at a $B C$ site to be $-1.05 \mathrm{eV}$ relative to a hydrogen atom in vacuo. With a $\mathrm{H}-\mathrm{H}$ bond energy, $E_{g}\left(\mathrm{H}_{2}\right)$, of $4.5 \mathrm{eV}$, we find $E(\mathrm{H})-E_{g}\left(\mathrm{H}_{2}\right) \sim 1.2 \mathrm{eV}$. Hence $[\mathrm{H}] \sim 5 \times 10^{19} \exp \left(-u_{1} / k T\right) \mathrm{cm}^{-3}$ with $u_{1} \sim 1.2 \mathrm{eV}$. On the other hand $E\left(\mathrm{H}_{2}\right)$ has energy about $0.6 \mathrm{eV}$ above that of the gas molecule and the molecular concentration is then given by $\left[\mathrm{H}_{2}\right] \sim 6 \times 10^{15} \exp \left(-u_{2} / k T\right) \mathrm{cm}^{-3}$ with $u_{2} \sim 0.6 \mathrm{eV}$.

At $1100^{\circ} \mathrm{C}$ the concentration of hydrogen atoms is about $3 \times 10^{15} \mathrm{~cm}^{-3}$, i.e. around 100 times the molecular concentration. If the ampoule is now cooled without loss of hydrogen, there is conversion of $\mathrm{H}$ into $\mathrm{H}_{2}$. Around $800^{\circ} \mathrm{C}$, there are equal quantities, but at room temperature all the hydrogen will be molecular. These results although very reasonable seem to differ from the available experimental data. 
When hydrogen is in-diffused into $\mathrm{Si}$ at temperatures $T_{i}$ between 900 and $1300{ }^{\circ} \mathrm{C}$ and the samples allowed to cool, it aggregates into molecules leaving a temperature dependent fraction as a rapidly diffusing monatomic species. Below about $200{ }^{\circ} \mathrm{C}$, this fraction can becomes trapped by boron forming B-H pairs. The complexes dissociate above this temperature. The concentration of $\mathrm{B}-\mathrm{H}$ pairs, and thus of trapped monatomic hydrogen, can be found from IR-absorption studies. The concentration of $\mathrm{H}$ found in this way depends on the solubility of hydrogen (and thus $T_{i}$ ) and is unaffected by the concentration of boron up to $10^{17} \mathrm{~cm}^{-3}$. SIMS measurements indicate that there is far more hydrogen in the sample than bound to boron consistent with the view that much is of molecular form.

Annealing below $200{ }^{\circ} \mathrm{C}$, increases the concentration of $\mathrm{B}-\mathrm{H}$ pairs. Around about $100{ }^{\circ} \mathrm{C}$, molecules will begin to diffuse and participate in the reaction $\mathrm{H}_{2}+2 \mathrm{~B} \rightarrow 2 \mathrm{~B}-\mathrm{H}$. According to calculations [31], this reaction is exothermic with $\Delta H$ about $-0.5 \mathrm{eV}$. Thus the concentration of $\mathrm{B}-\mathrm{H}$ pairs increases until all the molecules have been converted.

FTIR measurements on samples annealed below $200^{\circ} \mathrm{C}$ showed the the maximum concentration of hydrogen bound to B was consistent with SIMS measurements and is given by $9.1 \times 10^{21} \exp \left(u_{1} / k T_{i}\right) \mathrm{cm}^{-3}$, where $u_{1}=-1.8 \mathrm{eV}$. The pre-exponential constant and energy seem rather large here and both are larger than the the theoretical results. At $1100{ }^{\circ} \mathrm{C}$, the concentration of $\mathrm{H}, \sim 5 \times 10^{15} \mathrm{~cm}^{-3}$, is about the same as the theoretical value but the solubility is much lower at lower temperatures. This may reflect the fact that not all molecular hydrogen reacts with boron.

For quenched (unannealed) samples, the concentration of B-H pairs is much lower than this, and most of the hydrogen is in a molecular form. This is supported by recent observations which have detected $\nu_{3}$ modes due to $\mathrm{H}-\mathrm{H}$ molecules (see Section 7.1) in thick samples. Assuming that there is equilibrium between the monatomic and diatomic hydrogen species, then the equilibrium concentration of molecules can be found from the difference in the absorption intensities between quenched and annealed samples. Thus the molecular concentration is found to be $5.2 \times 10^{23} \exp \left(u_{2} / k T_{i}\right) \mathrm{cm}^{-3}, u_{2}=-2.4 \mathrm{eV}$. The pre-exponential factor and energy are very much larger than the theoretical estimates with the former being exceptionally large. This clearly shows the difficulty in measuring the molecular density. Further work is necessary to resolve these discrepancies. 
The hydrogen molecules can be broken up by irradiation and thermal treatments. Room temperature electron irradiation [156] of the quenched samples generated $\mathrm{H}_{2}^{*}$. showing that molecules are easily converted in presence of vacancies or interstitials consistent with modelling studies [153]. Curiously, no $\mathrm{V}_{n} \mathrm{H}_{m}$ defects were detected, and this is not understood.

In quenched thick samples where only $5 \%$ of the total hydrogen concentration was bound to boron, FTIR measurements [157] revealed that the $\nu_{3}$ band (Section 7.1) due to molecules was present. This is direct evidence that the 'hidden' hydrogen is molecular. Furthermore, the intensity of the $\nu_{3}$ band decreased with anneals at $160^{\circ} \mathrm{C}$, because of conversion to $\mathrm{B}-\mathrm{H}$ defects via the reaction:

$$
\mathrm{B}+\mathrm{H}_{2} \rightarrow \mathrm{BH}+\mathrm{H}
$$

The activation barrier for this reaction was found to be greater than the barrier for diffusion of $\mathrm{H}_{2}\left(0.78 \mathrm{eV}\right.$, see Section 7.3). Extended anneals at $170{ }^{\circ} \mathrm{C}$, did not succeed in removing all the molecular hydrogen and led to a loss of BH defects. This indicates that hydrogen was forming new defects as there was neither an increase in the $\nu_{3}$ band, nor hydrogen effusion from the sample. This shows that more stable defects are being formed. FTIR measurements showed that there is a growth in the intensity of a line at $2222 \mathrm{~cm}^{-1}$, already assigned to $\mathrm{VH}_{4}$ defects (Section 5.1). It is unclear whether the vacancies are produced from the break up of grown in defects, defects created by the quench, or created directly by hydrogen.

\subsection{Plasma Treatments}

Hydrogen concentrations obtained from low temperature plasmas $\left(<300^{\circ} \mathrm{C}\right)$ are $\sim 10^{20} \mathrm{~cm}^{-3}$ within a depth of around $0.1 \mu \mathrm{m}[143]$ of the surface. The hydrogen concentration at greater

depths is sensitive to doping type and penetrates B-doped silicon more easily than P-doped, presumably due to a greater number of traps. Many planar defects are then created lying on $\{111\}$ and $\{100\}$ planes [158]. These give vibrational bands around 1960 and $2100 \mathrm{~cm}^{-1}$ indicating that their inner surfaces are saturated with $\mathrm{Si}-\mathrm{H}$ bonds. They are not associated with dislocations, and therefore are generated by an opening of the lattice rather than a slip process. They are stable to about $350^{\circ} \mathrm{C}$ [159]. NMR studies show that, in deuterated material, they contain molecular $\mathrm{D}_{2}$ [102]. Plasma treated material also gives a broad band luminescence around $1 \mathrm{eV}$ and DLTS studies show the presence of deep $(-/ 0)$ electron traps within the 
band gap at $E_{c}-0.51$ and $E_{c}-0.06 \mathrm{eV}$. These traps have densities around $10^{12} \mathrm{~cm}^{-3}$ and are unrelated to the extended defects. It is likely that they are related to vacancy, or multivacancy, hydrogen defects. Leitch et al. [106] have presented evidence that a plasma treatment at above $150{ }^{\circ} \mathrm{C}$ generates platelets to which molecules subsequently diffuse. Thus molecules are a stable form of hydrogen within the lattice, but will diffuse to a void if their concentration exceeds the solubility limit.

There have been a number of theoretical attempts to predict the structure of platelets. Van de Walle et al. $[15,25]$, using DFT with 32 atom supercell calculations, considered pairs of $\mathrm{H}_{B C}^{0}$ in two adjacent $\mathrm{Si}-\mathrm{Si}$ bonds arrayed in the same (111) plane. They found a small lowering in energy when compared to isolated $\mathrm{H}_{B C}^{0}$ and similarly found that two $\mathrm{H}$ atoms placed to break a $\mathrm{Si}-\mathrm{Si}$ bond was also more stable. However, both configurations were higher in energy than molecular hydrogen in the lattice. Deák et al. [142] using MINDO/3 in 32 atom cyclic clusters, considered five models for platelets:

1. Groups of hydrogen molecules at adjacent $T$ sites.

2. Groups of $\mathrm{H}_{B C}^{0}$ in adjacent bonds along the same (111) plane.

3. Pairs of hydrogen atoms breaking a $\mathrm{Si}-\mathrm{Si}$ bond, so forming two $\mathrm{Si}-\mathrm{H}$ bonds.

4. Pairs of hydrogen atoms saturating the dangling bonds formed by removing a double layer of silicon atoms thus creating an array of $\left[\mathrm{V}_{2} \mathrm{H}_{2}\right]_{n}$ defects.

5. Groups of $\mathrm{H}_{2}^{*}$ defects (see Section 4) aligned in the same direction.

They considered that the third model, i.e. pairs of hydrogen atoms breaking a Si-Si bond forcing apart two (111) planes, was the most likely to be the defect responsible for platelets and calculated that an inter-planar separation of $5.92 \AA$ between the silicon atoms forced apart by the hydrogen was the most stable. Zhang et al. [160] also considered variations of the $\mathrm{H}_{2}^{*}$ structure used by Deák et al., using 24 atom supercells. They suggested that a double-layer $\{111\}$ platelet formed of alternating $\mathrm{H}_{2}^{*}$ units was $0.15 \mathrm{eV}$ lower in energy than interstitial $\mathrm{H}_{2}$ molecules.

Roberson and Estreicher [61] suggested that an aggregation of mobile vacancy-hydrogen complexes (see Section 5) could occur, leading to a platelet structure. However, it is unclear why the planar structure of the platelet would be formed in such a model.

Transmission electron microscopic studies have been carried out on (111) platelets [141] 
introduced by proton implantation followed by an anneal at $200{ }^{\circ} \mathrm{C}$. The TEM observations showed that the best model was one in which hydrogen displaced apart the (111) planes joined by $\langle 111\rangle$ orientated $\mathrm{Si}-\mathrm{Si}$ bonds by $3 \AA$. The analysis favoured the model of Deak et al. [142] where hydrogen saturated the Si dangling bonds on the two displaced surfaces.

\subsection{Wet etching}

Another method of incorporating $\mathrm{H}$ at low temperatures has been used with great success in a number of DLTS studies. Hydrogen is introduced via wet chemical etching (acid solution) at around room temperature $[161,162,163]$. The amount of hydrogen introduced is very low and is confined to the surface region. Much of the hydrogen is bound to $\mathrm{P}$ donors or B acceptors present in the material but annealing under a reverse bias (RBA) has the effect of driving the hydrogen deeper into the sample $[164,165,166,167,168,169]$. This method is particularly useful for a study of hydrogen related defects which dissociate around room temperature.

\section{Transition metal-hydrogen defects}

Si wafers are easily contaminated by transition metal (TM) impurities. This happens, for example, during heat treatments or when metallic contacts are added to devices. The main effect of the TM impurity is to introduce carrier traps and recombination centres. As unintentional contaminants $\mathrm{Fe}, \mathrm{Cu}$ or $\mathrm{Ni}$ are usually found in concentrations of $\sim 10^{12} \mathrm{~cm}^{-3}$ but, even in these minute amounts, they have measurable effects on minority carrier lifetimes.

It is now known that the electrical properties of a TM centre can be greatly modified by interaction with hydrogen. Hydrogenation of substitutional TM centres can shift the position of the deep levels due to the impurity $[164,165,166,167,168,169,170,171,172,173,174]$. Remarkably, the TM impurity can, in some cases, be completely passivated $[175,176,177]$. This, of course, has raised interest in the properties of TM-H defects although, disappointingly, the dissociation temperature of the complexes appears to be quite low [178]: the levels of isolated $\mathrm{Au}$ and $\mathrm{Pt}$ are reactivated under annealing around $200^{\circ} \mathrm{C}[168]$ and $330^{\circ} \mathrm{C}$ respectively [165].

Moreover, it is becoming apparent that levels reported many years ago are, or could be, due to TM-H defects. For example, DLTS measurements [179, 180, 181, 182] on Pt doped $n$-Si have revealed a deep electron trap, located near the surface, with a level at $\sim E_{c}-0.50 \mathrm{eV}$ and 
which plays an important part in limiting the lifetime of minority carriers. The assignment of this level has been very controversial and there are several suggestions of models for the defect responsible: Pt-Pt pairs [183, 184], Pt-O pairs [181, 185, 186] and Pt-vacancy. However, very recently, Sachse et al. [178] have argued that this mid-gap level arises from a defect involving Pt and hydrogen. This has created additional interest in the effects of $\mathrm{H}$ on the electrical levels of TM defects.

In this chapter, we review the experimental data on $\mathrm{TM}-\mathrm{H}$ defects and describe recent theoretical advances on these defects.

\subsection{Substitutional transition-metal defects}

As isolated impurities in $\mathrm{Si}$, transition metals show remarkable properties. They can possess very high diffusivities as exemplified by $\mathrm{Cu}$, which has an activation energy for diffusion similar to hydrogen. Its diffusivity between $400-900^{\circ} \mathrm{C}$ is $\sim 1.2 \times 10^{-4} \mathrm{~cm}^{2} \mathrm{~s}^{-1}$ while its solubility is $8 \times 10^{17} \mathrm{~cm}^{-3}$ at $1100^{\circ} \mathrm{C}$ [187]. TM impurities have large covalent radii and, like $\mathrm{H}$, readily react with dopants and other defects, as well as complexing with other TM centres [184, 188, 189, 190, 191, 192, 193]. They are readily attracted to aggregates of vacancies (micro-voids) and precipitate at stacking faults and along dislocations [194]. Their attraction to voids, created by irradiation, and located in the inactive regions of the device, can be used to control the effects of the TM impurities. This 'proximity gettering' is being intensively investigated [195, 196, 197]. Very recently, Au 'labelling' has been used to map the concentration of vacancy clusters in MeV-implanted Si [198].

Isolated TM impurities can sit interstitially, such as Fe [199, 200], or substitutionally like $\mathrm{Au}[201,202], \operatorname{Ag}[192,203]$, Pt [204, 205, 206] and Ni [207, 208]. There does not appear to be a simple theory which explains why one element prefers to sit at an interstitial site while another is a substitutional defect. Interstitial TM defects migrate rapidly and are able to find dopants to form centres such as Fe-B pairs, before they occupy substitutional sites via a kick-out mechanism as in the case of $\mathrm{Pt}[181,195,209,210]$.

EPR studies have revealed that isolated substitutional $\mathrm{Ni}_{s}^{-}[207,211,212,213], \mathrm{Pt}_{s}^{-}$[204, 206, 214, 215], $\operatorname{Pd}_{s}^{-}[204,207,212,213]$, and $\operatorname{Ag}_{s}^{0}[192,203]$ centres have orthorhombic symmetry $\left(C_{2 v}\right)$ in their paramagnetic charge states with a $S=\frac{1}{2}$ ground-state. The 'vacancy' model of 
Watkins [216] explains this distortion as arising from a close connection with the isoelectronic $\mathrm{V}^{-}$centre. Take as an example the substitutional $\mathrm{Pt}^{-}$and $\mathrm{Au}^{0}$ centres. The impurity $d$-levels are filled by ten electrons, lie deep in valence band and interact weakly with the $t_{2}$-states of the charged vacancy. The remaining electrons derived from the atomic configuration $5 d^{10} 6 s^{1}$ go into the vacancy related $t_{2}$-manifold (triplet) which is then occupied by three electrons (as in $\mathrm{V}^{-}$). In other words, a TM impurity ion is thought as a black-box which possesses electrical properties of a centre arising solely from the vacancy-like Si $s p^{3}$ hybrid orbitals. Calculations [217, 218, 219] support this model as the impurity $t_{2}$-levels were found to be energetically much lower than those of the Si dangling-bonds with which they interact. The partial occupancy of the $t_{2}$-level provides the basis for a Jahn-Teller distortion. This is similar to the lattice vacancy [220, 221] which undergoes a reconstruction through a pairing of the vacancy neighbours. The effect of this distortion is a splitting of the $t_{2}$-manifold into three close-by singlet levels.

Uniaxial stress measurements [222] on the EPR spectrum of $\mathrm{Pt}_{s}^{-}$have shown that the $t_{2}$ gap-levels split to form a $b_{1}^{\uparrow \downarrow} b_{2}^{\uparrow} a_{1}^{0}$ configuration, which is, however, the reverse of that of $\mathrm{V}^{-}$, i.e., $a_{1}^{\uparrow \downarrow} b_{1}^{\uparrow} b_{2}^{0}$ [221]. Anderson et al. [205] have demonstrated that if non-linear JT-coupling terms are considered, the vacancy model can account qualitatively for the sense of the JT distortion undergone by the $\mathrm{Pt}_{s}^{-}$centre. LDF calculations support the ordering of levels found in $\mathrm{Ni}_{s}^{-}$[223].

The vacancy model (which supersedes the ionic model of Ludwig and Woodbury [224]) postulates the transfer of electrons from the $d$-shell of the impurity into valence states to achieve strong dative bonding between the impurity ion and its four Si nearest-neighbours with Hund's rule being satisfied. This appears to be the case for $\mathrm{Ni}_{s}^{-}$in diamond where $S=\frac{3}{2}[225,226]$, but in $\mathrm{Si}$ where the lattice is softer, JT effects are much stronger.

Unexpectedly, the EPR spectrum of $\mathrm{Au}_{s}^{0}$ has never been observed. However, Zeeman luminescence studies on the $\mathrm{Au}_{s}^{+} \rightarrow \mathrm{Au}_{s}^{0}$ transition together with uniaxial stress measurements $[202,227]$ demonstrate that the $\mathrm{Au}$ centre exhibits similar properties to all the other TMs near the end of the $3 d, 4 d$ and $5 d$ series. Moreover, this study shows that spin-orbit effects are even more important for $\mathrm{Au}_{s}$ than for $\mathrm{Pt}_{s}$, with the $g$-tensor components of the former being $g_{x x}=g_{y y} \sim 0$ and $g_{z z}=2.8$. These are to be compared with $g_{x x}=1.38, g_{y y}=1.42$ and $g_{z z}=2.07$ for $\mathrm{Pt}_{s}^{-}$[204]. The vanishing of $g_{x x}$ and $g_{y y}$ in the case of $\mathrm{Au}$ then accounts for the absence of an EPR spectrum. These TM impurities show an ease of reorientation even 
at the lowest of temperatures $(T<4 \mathrm{~K})[206,215]$, suggesting a very small departure from $T_{d}$ symmetry. This is supported by modelling which has shown that the off-site displacement of the TM ion is $0.03 \AA$ for $\mathrm{Au}_{s}^{0}[30]$ and $0.05 \AA$ for $\mathrm{Pt}_{s}^{-}[228]$.

A characteristic of a TM impurity in silicon is a number of deep-lying levels within the Si bandgap which can trap carriers. In contrast with shallow level defects (like those of B, P, Ga, or $\mathrm{Al}$ ), which are characterised by effective mass like wavefunctions and small binding energies, TM levels are deep: sometimes around mid-gap and with wavefunctions which are localised. Deep levels are defined by the position of the Fermi level where the occupancy changes. For example, the $(-/ 0)$ acceptor level is the position in the gap above which the Fermi level must lie, for the defect to change from being neutral to negatively charged at zero-temperature. The same impurity may exhibit several deep levels. For example, $\mathrm{Cu}$ in $\mathrm{Ge}$ can act as a triple, double and single acceptor as well as a donor $[229,230]$. For this to happen it is necessary that the Mott-Hubbard Coulomb energy, $U$, responsible for the separation between the $(-/ 0)$ and $(0 /+)$ levels of a given defect, is extremely small. It has been argued that this arises from a change in hybridization with charge state which has the effect of distributing the additional electrons amongst the ligands. Thus the additional charge resides in the host lattice rather than on the impurity itself [231]. However, this argument does not take into account the change in the structure of the defect consequent upon a charge state change. These latter considerations are important in TM-H defects where for every $\mathrm{H}$ atom added, the electron occupancy of the $t_{2}$-manifold increases. The fact that the TM impurity can bind several electrons implies that several $\mathrm{H}$ atoms can also be added and significantly, as we shall see in Section 9.5, the H atoms do not bond with the TM impurity itself, except possibly in the defect with one $\mathrm{H}$ atom, but lie outside the vacancy cage.

TM impurities are introduced into Si either by direct doping of the melt during crystal growth or by evaporation of the metal at high temperatures onto the surface. Due to their high diffusivity and tendency to precipitate [187], only approximately $1 \%$ of the total metal content gives rise to electrically active centres.

Substitutional isolated gold, $\mathrm{Au}_{s}$, is an amphoteric centre giving rise to only two levels in the bandgap: a single acceptor level $0.56 \mathrm{eV}$ below the conduction band minimum $\left(E_{c}-0.56 \mathrm{eV}\right)$, and a single donor level $0.35 \mathrm{eV}$ above the valence band top $\left(E_{v}+0.35 \mathrm{eV}\right)[168] . \mathrm{Ag}_{s}$ shows a 
similar level structure, with the $(-/ 0)$ level at $E_{c}-0.55$ and the $(0 /+)$ level at $E_{v}+0.37 \mathrm{eV}[167]$. Substitutional Pt, $\mathrm{Pd}$ and $\mathrm{Ni}$ show a similar behaviour although in addition to the single acceptor and donor levels, $\mathrm{Pt}_{s}$ and $\mathrm{Pd}_{s}$, possesses a double donor level close to the valence band top $[165,232,233,234] . \mathrm{Ni}_{s}$ has a $(0 /+)$ level at $E_{v}+0.15$, a $(-/ 0)$ level at $E_{c}-0.39$ and a double acceptor level, (=/-), at $E_{c}-0.08 \mathrm{eV}[164]$.

Information in the literature on the activation energies and capture cross-sections of TM impurities determined by DLTS measurements suffers from discrepancies and inconsistencies. Thus the tabulated capture cross-sections of a given defect sometimes differ by orders of magnitude [235]. Capture cross-sections are sometimes thermally activated which imply that the activation energy measured by DLTS is not simply the thermodynamic energy level. This can cause difficulties in reconciling theory with experiment. In addition, the emission of carriers from bistable defects may give information on the levels of a metastable defect configuration and are not directly related to thermodynamic ones. The di-carbon centre in Si is an excellent example of this [236]. Finally, a serious problem confronting theoretical modelling is that the electrical character of the level is not always reported. Thus whether a transition is $(0 /+)$ or $(-/ 0)$ is in many cases unknown. The magnitude of the cross-section can give a guide to the character: those of second acceptors and donors are usually an order of magnitude smaller than the single acceptors or donors because of the Coulomb barrier encountered in the reactions:

$$
\begin{aligned}
& D^{+}+h^{+} \rightarrow D^{2+} \\
& D^{-}+e^{-} \rightarrow D^{2-}
\end{aligned}
$$

However, there appear to some exceptions to this as in the cross-sections for the $(=/-)$ and $(-/ 0)$ transitions in $\mathrm{V}_{2}$ [237]. Additional help in the assignment of the character of the transition comes from the field dependence of the emission rate. Emission of electrons by single acceptor levels in the upper half of the band gap is not expected to be sensitive to a electrical field because the final state is neutral. This, however, is not the case when the transition arises from a donor where a large Poole-Frenkel effect is to be expected. A smaller effect occurs for double acceptors. Analogous arguments can be given for hole transitions to donors in the lower half of the band gap. These aspects show that the analysis of a DLTS spectrum is rarely ever straightforward. 
DLTS can also provide information on defect concentration as a function of depth. If two different transitions have the same depth profile, it is tempting to argue that they arise from the same defect. This is particularly important as it is rarely the case that theoretical modelling is able to give sufficiently precise information to assign a defect based on a single DLTS transition. If several transitions have been assigned then the possibility for error is reduced. However, if one transition is masked by another defect, then the method may be misleading. An example of this are the $(-/ 0)$ levels of $\mathrm{AuH}_{2}$ and $\mathrm{AuH}_{1}$ (as well as the $\mathrm{Ag}$ analogues) which are particularly close and whose profiles have caused confusion. Another example is an early DLTS study of $\mathrm{Au}_{s}$ by Lang et al. [238] in which the traps at $E_{c}-0.56$ and $E_{v}+0.35 \mathrm{eV}$ were incorrectly assigned to different defects [201]. Unfortunately, DLTS does not provide any direct information about the structure or chemical composition of the defect.

The application of uniaxial stress can lead to information on the symmetry of the defect which is extremely useful in its identification. This technique has been applied [239] to the $A$-centre, or $\mathrm{V}-\mathrm{O}$ centre in $\mathrm{Si}$, which is known to have $C_{2 v}$ symmetry [240]. To our knowledge, this technique has only been applied to investigate the symmetry of one TM-related centre in silicon [241].

It is to be hoped that theory may also provide useful information on TM-related centres and in the next section, we summarise the available theoretical tools for the study of their properties and in particular their electrical levels.

\subsection{Modelling deep-level TM centres}

Pioneering studies of TM defects in Si were carried out by Cartling [218] and Hemstreet [216, 217, 242, 243] using a self-consistent $X \alpha$-cluster formalism. Although the clusters used were very small and no structural relaxation was included, the method served to demonstrate that the impurities introduce gap levels, and that their wavefunctions were mainly localised on $\mathrm{Si}$ atoms. These studies then laid the foundation for the vacancy model. Next, more sophisticated methods were introduced to determine the energy levels of TM substitutional defects. These included the use of Green-function methods within the density-functional formalism [219, 244, $245,246,247,248,249,250,251,252,253,254,255]$.

All these different approaches have provided information on the properties of TM impurities 
in Si but with a relatively large error bar. The source of this error varies from one study to another, either being due to the use of a very small clusters, lack of spin polarisation, poor description of correlation effects or, the absence of structural optimisation. The inclusion of the latter is of course essential in the understanding of JT distortions [221, 256, 257, 258].

Of particular interest here are the donor and acceptor levels associated with TM-related defects. The position of these levels within the bandgap are usually calculated as the energy difference between a defect state and the band-edge. Consider the process involving the capture of an electron in the conduction band by the $t_{2}$ manifold of a neutral impurity centre. The corresponding change in the total energy $\left(E_{T}\right)$ of the system leads to the $(-/ 0)$ level given by

$$
E_{c}-E(-/ 0) \equiv E_{T}\left[t_{2}^{n} ;(\mathrm{CB})^{1}\right]-E_{T}\left[t_{2}^{n+1} ;(\mathrm{CB})^{0}\right]
$$

A practical disadvantage in using this expression to evaluate the $(-/ 0)$ level lies in the fact that the energy change due to the change in occupancy of two levels is very small compared with the total energy. However, Slater's transition-state method [259] allows a much more accurate calculation of this energy difference. This assumes that $E_{T}$ is a continuous function of the occupation numbers of $n$ electrons in the $t_{2}$-manifold and in the conduction band $(\mathrm{CB})$ respectively, and therefore each of the above expressions can be found from a Taylor expansion about the transition state defined by the configuration $\left[t_{2}^{n+1 / 2} ;(\mathrm{CB})^{1 / 2}\right]$. The difference in total energies of the two configurations is then related to the difference in the derivatives of $E_{T}$ with occupancy of the $t_{2}$-manifold and the CB. These derivatives, by Janak's theorem [260] are simply the Kohn-Sham energy levels. Thus,

$$
E(-/ 0) \sim E_{c}-E_{t_{2}}
$$

where $E_{t_{2}}$ is the Kohn-Sham eigenvalue corresponding to the $t_{2}$-manifold, with the electronic configuration of the transition state. The energy level of the CB is taken to be independent of configuration.

Similarly, the donor level can be related to the difference in $E_{t_{2}}$ and $E_{v}$ in the transition state configuration corresponding to an occupancy $t_{2}^{n-1 / 2}$. A problem is that none of the ab initio methods are able to properly describe simultaneously the localised defect state and the extended $\mathrm{CB}$ and in particular the energy gap. To circumvent this, the band gap calculated by the 
Table 10. Calculated donor and acceptor levels for substitutional TM impurities in silicon. $(0 /+)$ is referred to $E_{v}$ and, $(-/ 0)$ and $(=/-)$, to $E_{c}$. Values are in $\mathrm{eV}$.

\begin{tabular}{cccccccc}
\hline \hline & \multicolumn{2}{c}{$(0 /+)$} & \multicolumn{2}{c}{$(-/ 0)$} & \multicolumn{2}{c}{$(=/-)$} & \\
& Calc. & Obs. & Calc. & Obs. & Calc. & Obs. & Refs. \\
\hline \multirow{2}{*}{$\mathrm{Au}$} & 0.56 & 0.35 & 0.28 & 0.56 & & & {$[252]$} \\
$\mathrm{Ag}$ & 0.50 & 0.37 & 0.4 & 0.55 & & & {$[252]$} \\
$\mathrm{Ni}$ & 0.18 & 0.15 & 0.30 & 0.39 & 0.03 & 0.08 & {$[254]$} \\
$\mathrm{Co}$ & 0.30 & 0.40 & 0.19 & 0.40 & 0.14 & $\ldots$ & {$[254]$} \\
\hline \hline
\end{tabular}

method is either scaled or a scissors operator [261] is used to bring it into alignment with the experimental one. This method has been applied to calculate the levels of a number of substitutional isolated TM centres in Si with considerable success (Table 10).

Very recently, a new approach to this problem has been introduced [30]. To circumvent the difficulty of describing the band gap, the electron affinity of the defect $A_{d}$ is calculated. This is simply the difference in energies between a charged and neutral defect which again can be calculated by a Taylor expansion about a relaxed transition state configuration $t_{2}^{n+1 / 2}$. If the equilibrium structures of the neutral, charged and transition states are denoted by $\mathbf{R}^{0}, \mathbf{R}^{-}$, and $\mathbf{R}^{t}$ respectively, then

$$
\begin{aligned}
A_{d} & =E_{T}\left(t_{2}^{n}, \mathbf{R}^{0}\right)-E_{T}\left(t_{2}^{n+1}, \mathbf{R}^{-}\right) \\
& \simeq-E_{t_{2}}-\frac{\partial^{2} E_{T}\left(t_{2}^{n+1 / 2}, \mathbf{R}^{t}\right)}{\partial n \partial \mathbf{R}^{t}} \cdot\left(\mathbf{R}^{-}-\mathbf{R}^{0}\right) .
\end{aligned}
$$

Here $E_{t_{2}}$ is the Kohn-Sham eigenvalue associated with the $t_{2}$-manifold in the transition state. The second term can be ignored if the difference in the structures of the neutral and charged states of the defect are small. The affinity is then related to the Kohn-Sham level of the defect in a transition state. However, the calculation of this quantity is subject to the same difficulties as described above. For example, the electron affinity of bulk Si is simply the band gap which cannot be calculated with sufficient precision. However, we can compare the electron affinities for two defects having localised states within the band gap. Their difference being total energy differences, are simply their relative acceptor levels. In practice, the carbon interstitial defect, $\mathrm{C}_{i}$, which possesses $(-/ 0)$ and $(0 /+)$ levels at $E_{c}-0.1$ and $E_{v}+0.28 \mathrm{eV}$ respectively [236] is used as a standard. For second acceptor levels, the $(=/-)$ level of $\mathrm{PtH}_{2}$ is used (see Section 9.3 below), which is taken to lie at $E_{c}-0.15 \mathrm{eV}[262]$. 
The acceptor and donor levels of the defect, are then given by:

$$
(-/ 0)_{d}=(-/ 0)_{s}+A_{s}-A_{d} \quad \text { and } \quad(0 /+)_{d}=(0 /+)_{s}+I_{s}-I_{d}
$$

where $I_{d}$ and $I_{s}$ are the calculated ionisation energies of the defect and the standard.

The error associated with this method depends on how well the asymptotic part of the wavefunction of the ionised defect compares with that of the standard defect. The method works best when the defect levels are close to those of the standard.

Calculations using spin-polarised density functional cluster theory have been carried out for the standard defect $\mathrm{C}_{i}$ centre, $\mathrm{C}_{i}-\mathrm{P}$, the $T$-centre $\left(\mathrm{C}_{s}-\mathrm{C}_{i} \mathrm{H}\right), \mathrm{H}$ at a bond centred site $\left(\mathrm{H}_{B C}\right)$, $\mathrm{H}$ at an anti-bonded lattice site, $\mathrm{H}_{A B}, \mathrm{VO}, \mathrm{VP}, \mathrm{V}_{2}$, and $\mathrm{C}_{i}$. The results are given in Table 11 where they are compared with experimental values.

Table 11. Calculated [30] and observed electrical levels of deep centres. $(0 /+)$ is referred to $E_{v}$ and $(-/ 0)$ to $E_{c}$. Values are in $\mathrm{eV}$.

\begin{tabular}{lccccr}
\hline \hline & \multicolumn{2}{c}{$(0 /+)$} & \multicolumn{2}{c}{$(-/ 0)$} \\
& Calc. & Obs. & Calc. & Obs. & Refs. \\
\hline $\mathrm{VO}$ & 0.00 & $\ldots$ & 0.29 & 0.18 & {$[240]$} \\
$\mathrm{VP}$ & 0.43 & $\ldots$ & 0.44 & 0.43 & {$[263]$} \\
$\mathrm{V}_{2}\left(C_{2 h}\right)$ & 0.42 & 0.23 & 0.51 & 0.43 & {$[237]$} \\
$\mathrm{HC}_{i}-\mathrm{C}_{s}$ & 0.24 & $\ldots$ & 0.20 & 0.20 & {$[264]$} \\
$\mathrm{C}_{i} \mathrm{P}$ & 0.36 & 0.48 & 0.60 & 0.38 & {$[265]$} \\
$\mathrm{H}_{B C}$ & 0.94 & 1.00 & $\ldots$ & $\ldots$ & {$[38]$} \\
$\mathrm{H}_{A B}$ & $\ldots$ & $\ldots$ & 0.78 & $0.56^{*}$ & {$[49,29]$} \\
$\mathrm{V}_{2} \mathrm{O}$ & $\ldots$ & $\ldots$ & 0.47 & $\ldots$ & \\
$\mathrm{Pd}$ & 0.32 & 0.31 & 0.29 & 0.22 & {$[233,266]$} \\
$\mathrm{Pt}$ & 0.10 & 0.33 & 0.35 & 0.23 & {$[165,201]$} \\
$\mathrm{Ag}$ & 0.46 & 0.37 & 0.60 & 0.55 & {$[167]$} \\
$\mathrm{Au}$ & 0.21 & 0.35 & 0.66 & 0.56 & {$[168,201]$} \\
\hline \hline
\end{tabular}

* Muon spin-resonance experiments.

In general the calculated electrical levels of light impurities are within about $0.2 \mathrm{eV}$ of the experimental results where available. Whereas the donor and acceptor levels of $\mathrm{C}_{s}-\mathrm{C}_{i} \mathrm{H}, \mathrm{C}_{i} \mathrm{P}$, VP, VO and even $\mathrm{V}_{2}$ are within $0.2 \mathrm{eV}$ of the experimental values, it is remarkable that the $(0 /+)$ level of $\mathrm{H}_{B C}$ is found so close to the observed level given that the theory is expected to be worse when the level moves far away from that of $\mathrm{C}_{i}$. Inverted levels are found for hydrogen.

The theory is now used in Section 9.3 to describe the electrical levels of TM-H defects. 


\subsection{Theoretical Results}

$\mathbf{A u}-\mathbf{H}$ and $\mathbf{A g}-\mathbf{H}$ defects The electrical levels of various combinations of transition metalhydrogen defects containing several hydrogen atoms in Si have been calculated using spinpolarised local density functional cluster theory as described above. There are two likely sites for $\mathrm{H}$ next to the TM impurity. Either $\mathrm{H}$ lies (a) outside the vacancy or, (b) inside with approximate trigonal symmetry (Fig. 10). The energy differences between these configurations is not large for $n=1$ but the outside configuration is favoured for $n>1$. For example, in the case of $\mathrm{AuH}_{1}$, the energy of $\mathrm{H}$ anti-bonded to a Si neighbour ( $A B$ site) is lower by $0.23 \mathrm{eV}$ than when $\mathrm{H}$ lies inside the vacancy at an $A B$ site.

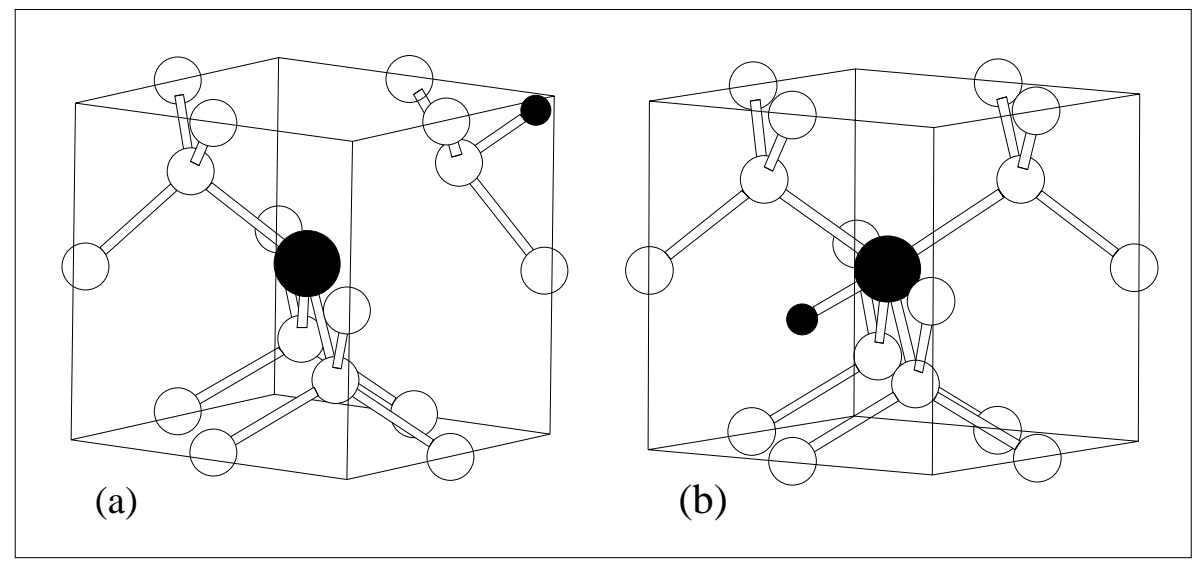

Fig. 10. Structure of TM- $\mathrm{H}_{1}$ complexes $\left(C_{1 h}\right)$ with $\mathrm{H}$ at anti-bonding $(A B)$ sites, outside (a) and inside the vacancy (b), according to theoretical calculations [228]. Cube axes lie along $\langle 100\rangle$ directions.

The $\mathrm{H}$ stretch vibrational frequencies for the three configurations are given in Table 12 along with experimental results [267]. Agreement is best for the configuration (a) but the sense of the small shifts arising with different charge states are not reproduced. The H-reorientation barrier among the equivalent $\langle 111\rangle$ directions is $0.41 \mathrm{eV}$ for (a) and $0.23 \mathrm{eV}$ for (b). The observed barrier is athermal and presumably must proceed by a tunnelling mechanism.

For $\mathrm{AuH}_{2}$ and $\mathrm{AgH}_{2}$ defects with $C_{2 v}$ symmetry, configuration (a) is more stable than (b) [30]. This also is the case for TM- $\mathrm{H}_{n}$ defects with $n>2$ (Figs. 11 and 12). This structure is the same as that suggested by magnetic resonance on $\mathrm{PtH}_{2}$ defects [268]. $\mathrm{AuH}_{2}$ defects have been detected by IR-absorption studies [267, 269]. Calculations [30] have shown that the separation in frequencies between the symmetric $\left(A_{1}\right)$ and antisymmetric $\left(B_{1}\right)$ modes for configuration (a) is much closer to experiment than the separations in configuration (b). 


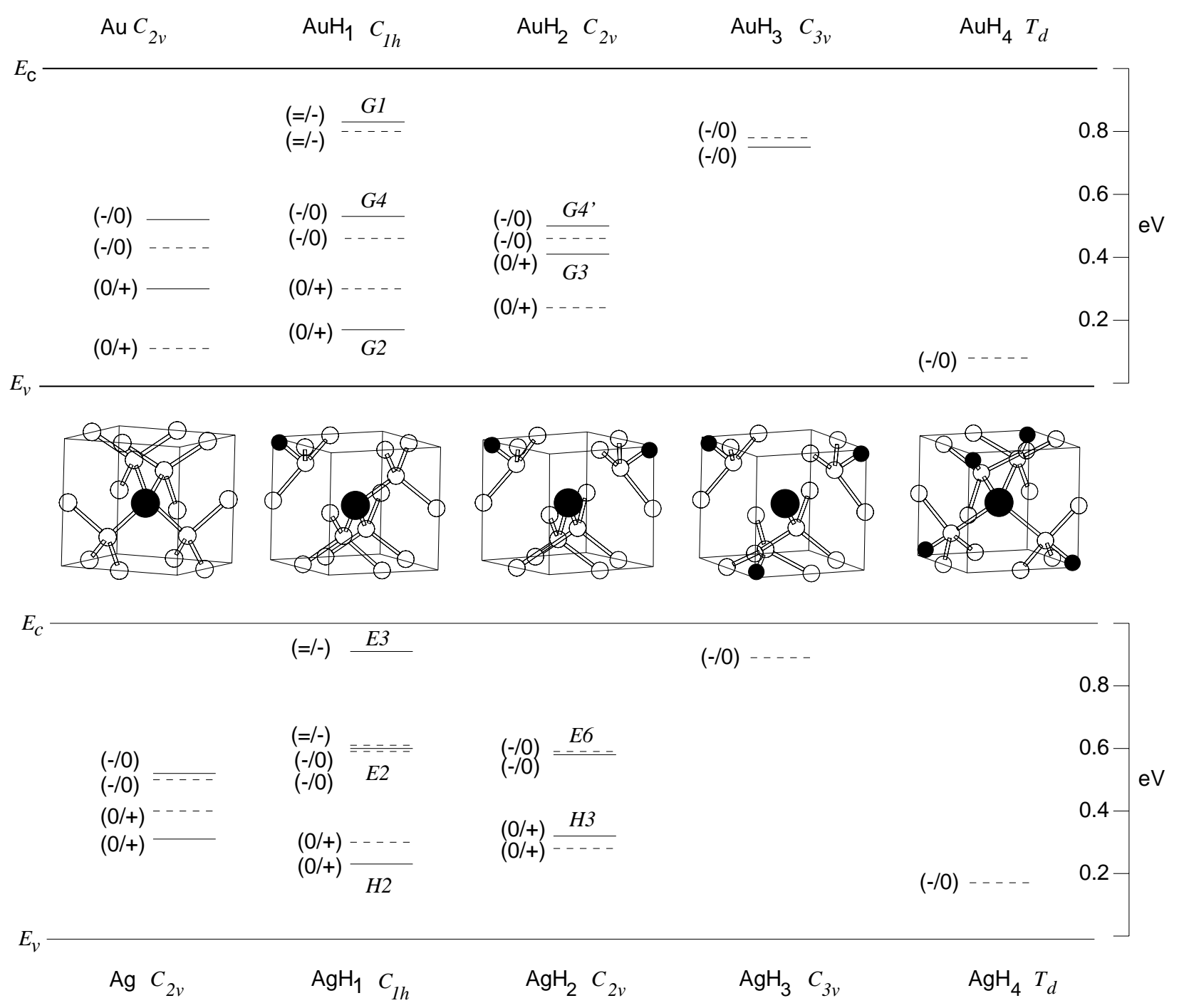

Fig. 11. Structure and corresponding electrical levels of gold and silver and their complexes with hydrogen in Si according to theoretical calculations [30, 228]. Experimental and calculated levels are represented by solid and dashed lines, respectively. The $\mathrm{AuH}_{3}$ assignment is tentative (Section 9.6).

Table 12. Calculated [30] and observed [267] hydrogen and deuterium stretch modes $\left(\mathrm{cm}^{-1}\right)$ for two configurations (see text) of $C_{1 h} \mathrm{AuH}_{1}$, in charge state $q$. The observed values are assumed to correspond to the given charge states.

\begin{tabular}{lcccccccccc}
\hline \hline & & \multirow{3}{*}{$q=0$} & & \multicolumn{3}{c}{$q=-1$} & & \multicolumn{3}{c}{$q=-2$} \\
\cline { 6 - 8 }$\left(\mathrm{AuH}_{1}\right)^{q}$ & $(\mathrm{a})$ & $(\mathrm{b})$ & Obs. & $(\mathrm{a})$ & (b) & Obs. & (a) & (b) & Obs. \\
\hline $\mathrm{AuH}_{1}$ & 1947.4 & 2014.5 & 1787.7 & 1890.5 & 1980.0 & 1813.3 & 1893.2 & 2118.0 & 1827.1 \\
$\mathrm{AuD}_{1}$ & 1401.1 & 1427.3 & 1292.9 & 1359.6 & 1402.8 & 1310.9 & 1361.4 & 1500.2 & 1319.4 \\
\hline \hline
\end{tabular}

The electrical levels are determined assuming that a low spin state results from the addition of each $\mathrm{H}$ atom, which lowers and splits the $t_{2}$ level into a filled $a_{1}$ level lying below a half-filled $e$-level. The lowering of the $t_{2}$-manifold is off-set by the splitting so that the differences in 


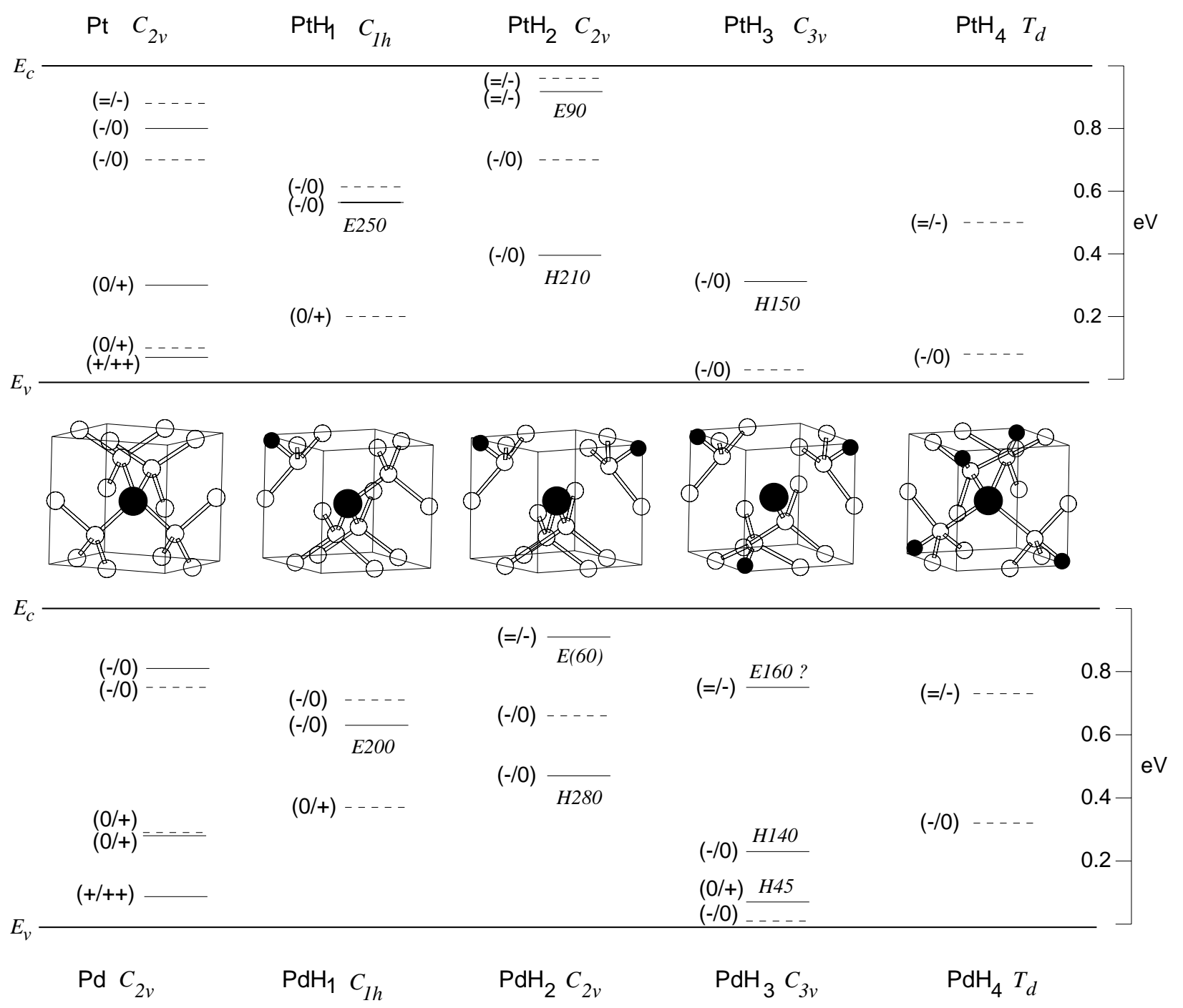

Fig. 12. Structure and corresponding electrical levels of platinum and palladium and their complexes with hydrogen in Si according to theoretical calculations [30, 228]. Experimental and calculated levels are represented by solid and dashed lines, respectively.

the donor and acceptor levels from those of the TM impurity are quite small. Table 13 gives these levels for configuration (a). The levels found for configuration (b) are very similar. For example, the $(0 /+),(-/ 0)$ and $(=/-)$ levels of $\mathrm{AuH}_{1}$ lie at $E_{v}+0.37, E_{c}-0.76$ and $E_{c}-0.45 \mathrm{eV}$ respectively.

A second $\mathrm{H}$ atom, added in configuration (a), results in an additional electron occupying the e-manifold which is pushed downward and splits with the upper level being occupied. Thus once again there are only small shifts in the donor and acceptor levels on going from say $\mathrm{AgH}_{1}$ to $\mathrm{AgH}_{2}$. The e-manifold is filled for $\left(\mathrm{AuH}_{2}\right)^{-}$and $\left(\mathrm{AgH}_{2}\right)^{-}$, and a second acceptor level can only arise from a new state entering the gap. The calculations give no hint of such states and hence the di-hydrogen defects do not possess $(=/-)$ levels. 
Table 13. Calculated [30, 228] and observed electrical levels of substitutional $\mathrm{Au}, \mathrm{Ag}, \mathrm{Pd}$ and $\mathrm{Pt}$ isolated centres and their complexes with hydrogen. $(0 /+)$ is referred to $E_{v}$ and, $(-/ 0)$ and $(=/-)$, to $E_{c}$. Values are in $\mathrm{eV}$.

\begin{tabular}{|c|c|c|c|c|c|c|c|}
\hline & \multicolumn{2}{|c|}{$(0 /+)$} & \multicolumn{2}{|r|}{$(-/ 0)$} & \multicolumn{2}{|r|}{$(=/-)$} & \multirow[b]{2}{*}{ Refs. } \\
\hline & Calc. & Obs. & Calc. & Obs. & Calc. & Obs. & \\
\hline $\mathrm{Au}$ & 0.21 & 0.35 & 0.66 & 0.56 & & & {$[168,201]$} \\
\hline $\mathrm{AuH}_{1}$ & 0.36 & $0.21(\mathrm{G} 2)$ & 0.62 & $0.54(\mathrm{G} 4)$ & 0.29 & $0.19(\mathrm{G} 1)$ & {$[166,168,172]$} \\
\hline $\mathrm{AuH}_{2}$ & 0.28 & 0.47 (G3) & 0.62 & $0.58\left(\mathrm{G} 4^{\prime}\right)$ & 0 & & {$[166,168,172]$} \\
\hline $\mathrm{AuH}_{3}$ & 0.00 & $\cdots$ & 0.26 & 0.28 & & & {$[173]$} \\
\hline $\mathrm{AuH}_{4}$ & $\ldots$ & $\cdots$ & $1.40^{\dagger}$ & $\cdots$ & & & \\
\hline $\mathrm{Ag}$ & 0.46 & 0.37 & 0.60 & 0.55 & & & [167] \\
\hline $\mathrm{AgH}_{1}$ & 0.36 & $0.28(\mathrm{H} 2)$ & 0.45 & $0.45(\mathrm{E} 2)$ & 0.45 & 0.09 (E3) & [167] \\
\hline $\mathrm{AgH}_{2}$ & 0.33 & $0.38(\mathrm{H} 3)$ & 0.50 & $0.5(\mathrm{E} 6)$ & 0 & & [167] \\
\hline $\mathrm{AgH}_{3}$ & 0.00 & $\ldots$ & 0.13 & $\ldots$ & & & \\
\hline $\mathrm{AgH}_{4}$ & 0.00 & $\cdots$ & 0.97 & $\ldots$ & & & \\
\hline $\mathrm{Pt}$ & 0.30 & 0.33 & 0.31 & 0.23 & 0.12 & & {$[165,201]$} \\
\hline $\mathrm{PtH}_{1}$ & 0.44 & $\ldots$ & 0.51 & $0.50(\mathrm{E} 250)$ & 0 & $\cdots$ & {$[165]$} \\
\hline $\mathrm{PtH}_{2}$ & 0 & $\ldots$ & 0.42 & $0.76(\mathrm{H} 210)$ & $0.15^{\ddagger}$ & 0.18 (E90) & {$[165,166]$} \\
\hline $\mathrm{PtH}_{3}$ & 0 & $\ldots$ & $1.35^{\dagger}$ & $0.90(\mathrm{H} 150)$ & 0 & $\ldots$ & [165] \\
\hline $\mathrm{PtH}_{4}$ & 0 & $\cdots$ & 1.14 & $\cdots$ & 0.54 & $\cdots$ & \\
\hline $\mathrm{Pd}$ & 0.52 & 0.31 & 0.25 & 0.22 & & & {$[233,266]$} \\
\hline $\mathrm{PdH}_{1}$ & 0.62 & $\cdots$ & 0.30 & $0.43(\mathrm{E} 200)$ & 0 & & {$[266]$} \\
\hline $\mathrm{PdH}_{2}$ & 0 & $\ldots$ & 0.36 & $0.61(\mathrm{H} 280)$ & 0 & $0.10(\mathrm{E} 60)$ & {$[266]$} \\
\hline $\mathrm{PdH}_{3}$ & 0 & $0.07(\mathrm{H} 45)$ & $1.15^{\dagger}$ & $0.93(\mathrm{H} 140)$ & 0 & $0.29(\mathrm{E} 160)^{*}$ & {$[266]$} \\
\hline $\mathrm{PdH}_{4}$ & 0 & $\cdots$ & 0.71 & $\cdots$ & 0.23 & $\cdots$ & \\
\hline
\end{tabular}

$\dagger$ - the method has a larger error when the predicted level is further away from that of the standard.

$\ddagger$ - this is the standard level, as discussed in the text.

* - this assignment is tentative.

Adding a third $\mathrm{H}$ fills the $e$-manifold which now falls below $E_{v}$. Thus $\mathrm{AgH}_{3}$ and $\mathrm{AuH}_{3}$ do not possess any donor levels. However, it appears that an empty level, due to the $5 s$ and $6 s$ levels of $\mathrm{Ag}$ and $\mathrm{Au}$ respectively, creeps into the band gap. The resulting (-/0) levels of $\mathrm{AgH}_{3}$ and $\mathrm{AuH}_{3}$ are placed at 0.13 and $0.26 \mathrm{eV}$ below $E_{c}$.

$\mathbf{P t}-\mathbf{H}$ and $\mathbf{P d}-\mathbf{H}$ defects For $\mathrm{PtH}_{1}^{0}$, the vacancy-like $e$-doublet contains one electron and is split by the reduced symmetry due to the presence of the proton. The splitting lowers the acceptor level and this is exaggerated by a further lowering due to the Coulomb potential of the proton. Thus the calculations find the $(-/ 0)$ level of $\mathrm{PtH}_{1}$ lies deeper in the gap than that 
of Pt. A second acceptor level lies close to $E_{c}$. In $\mathrm{PtH}_{1}^{+}$, the e-level is empty and the donor level is slightly deeper than that of Pt.

The addition of a second $\mathrm{H}$ atom results in an additional electron occupying the $e$-manifold, which is now half-full. There is only a slight upward shift in energy of the acceptor level in going from $\mathrm{PtH}_{1}$ to $\mathrm{PtH}_{2}$. This might make it difficult to resolve these two levels. There is no donor level as the $(0 /+)$ level lies in the valence band. We cannot calculate the $(=/-)$ level as we use this level as a standard.

For $\mathrm{PtH}_{3}^{0}$, the e-manifold contains three electrons and could trap a single electron. The calculation finds the $(-/ 0)$ level to be exceptionally deep at $E_{c}-1.35 \mathrm{eV}$. Clearly, in this case the shift from the level of the standard defect makes this estimate prone to error. However, there is evidence from DLTS studies (Section 9.6) that $\mathrm{PtH}_{3}$ or $\mathrm{PtH}_{4}$ possess a (-/0) level at $E_{c}-0.9 \mathrm{eV}$. A second acceptor can only arise from a non-vacancy like state, possibly arising from the $6 s$ orbital entering the bandgap. A similar situation occurs for the single acceptor level, $(-/ 0)$ of $\mathrm{PtH}_{4}$. This lies deep in the gap close to $E_{v} . \mathrm{PtH}_{2}$ and $\mathrm{PtH}_{3}$ do not possess any donor levels. Table 13 shows that $\mathrm{PdH}_{n}$ and $\mathrm{PtH}_{n}$ defects possess similar levels. None of the defects investigated are passive.

We now turn to the experimental investigations of the structure and energy levels of the defects.

\subsection{Experimental Techniques}

Several hydrogenation methods are currently used to investigate the interaction of $\mathrm{H}$ with TM centres in Si. Historically, the first was introduced by Pearton and co-workers [176, 270], following the pioneering work of Benton et al. [271] on the passivation of point defects created by laser illumination. Hydrogen is introduced into bulk silicon by subjecting the samples to a remote, low pressure, H-plasma in the range $200-300^{\circ} \mathrm{C}$. This technique is quite aggressive, leading to voids and platelets (see Section 8.2) and other native defects [272]. The distribution of $\mathrm{H}$ is rather inhomogeneous with concentrations of order $10^{20} \mathrm{~cm}^{-3}$ near the surface. As a consequence, $\mathrm{H}$ can only be found, in appreciable concentration, in the vicinity of the surface of the Schottky or $p$ - $n$ junction diode. Wet etching has also been used to great effect in DLTS studies. 
The heating of the sample in $\mathrm{H}_{2}$-gas at high temperatures, usually between 1200 and $1300{ }^{\circ} \mathrm{C}$ followed by rapid quenching to room temperature is another method of incorporating $\mathrm{H}$ (see Section 8.2). This hydrogenation process has been tailored for EPR or IR absorption studies which require an uniform density typically $\sim 10^{15} \mathrm{~cm}^{-2}[268,273]$. This method has been successfully applied to the study of $\mathrm{Pt}-\mathrm{H}$ and $\mathrm{Au}-\mathrm{H}$ complexes $[267,268,269,274]$.

Three techniques have been used to study the interaction of $\mathrm{H}$ with transition-metal impurities in crystalline silicon. These are EPR and IR absorption experiments which give information on the composition and structure of the defect, while DLTS gives information on the electrical levels. However, it has been difficult to cross-correlate these studies. It seems essential to include information from other studies such as minority-carrier transient spectroscopy (MCTS) [171] and Laplace DLTS (LDLTS) $[172,173]$. MCTS permits the measurement of the capture of electrons by hole traps in the lower part of the bandgap, or the capture of holes by electron traps near the conduction band edge. This combined with DLTS allows both acceptor and donor levels of the defect to be found in the same sample. Similarly, LDLTS, is able to resolve close-by electrical levels which distinguishes it from conventional DLTS. It has been successfully used to discriminate the $(-/ 0)$ levels of $\mathrm{AuH}_{1}$ and $\mathrm{AuH}_{2}$ which lie very close together and cannot be resolved in conventional DLTS. In LDLTS, the observed transient is measured as a function of time at a fixed temperature, and may be very useful for defects which diffuse rapidly or change their structure with temperature.

A principal problem with DLTS is that the numbers of atoms, and the chemical composition in a defect cannot be obtained directly. Recently, Feklisova and Yarykin [275] have suggested that the dependence of the defect concentration on depth might be used to identify the chemical composition of the defect. Assuming that multi-H defects are formed by diffusion of $\mathrm{H}$ from the surface, which is varying during the etching process, and successive capture of $\mathrm{H}$ by the defect, the authors argued that the concentration-depth dependence of a $\mathrm{TM}-\mathrm{H}_{n}$ defect, containing $n$ $\mathrm{H}$ atoms, is

$$
\left[\mathrm{TM}-\mathrm{H}_{n}\right] \propto \exp \left(-x L_{n}\right) \quad \text { and } \quad L_{n}=\frac{L}{n}
$$

provided $\left[\mathrm{TM}-\mathrm{H}_{n}\right] \gg\left[\mathrm{TM}-\mathrm{H}_{n+1}\right]$. Here, $x$ is the distance to the surface and $L_{n}$ is a characteristic penetration depth of the $\mathrm{TM}-\mathrm{H}_{n}$ defect, which depends on the diffusivity, lifetime and drift 
velocity of $\mathrm{H}$. If this equation is correct, then the slope of the concentration profiles gives $n$ the number of $\mathrm{H}$ atoms in the defect. However, an application of the method to $\mathrm{VOH}_{2}$ defects is controversial [276].

\subsection{Structural properties of TM-H defects}

Despite the obvious technological and scientific importance of complexes involving TM impurities and hydrogen in Si, details on their structure are rather sparse. Early studies were made of $\mathrm{CuH}_{2}$ complexes in germanium [277] and TM-H defects in bulk III-V crystals [278]. Clerjaud et al. [278] analysed the vibrational properties of TM-H complexes by means of LVM absorption measurements demonstrating the utility of the method.

Recently a combined EPR and IR absorption study of $\mathrm{PtH}_{2}$ defects has led to a detailed model of the defect $[222,267,268,269,274]$. LVM spectroscopy on samples subjected to a uniaxial stress, together with EPR studies, demonstrated that $\mathrm{PtH}_{2}^{-}$is a spin $\frac{1}{2}$ defect possessing $C_{2 v}$ symmetry with two equivalent $\mathrm{H}$ atoms. $\mathrm{H}$ related antisymmetric and symmetric modes at 1888.2 and $1901.6 \mathrm{~cm}^{-1}$ were correlated with the EPR activity. Additional H-vibrations were detected and assigned to other non-paramagnetic charge states of the same $\mathrm{PtH}_{2}$ defect. These are given in Table 14. The observations of only one $\mathrm{H}$ related mode in the mixed H/D isotopic case also shows that both $\mathrm{H}$ atoms are equivalent. Furthermore, the analysis of the anisotropic part of the hyperfine tensor, located the $\mathrm{H}$ atom at a distance of $\sim 4.2 \AA$ from the Pt ion lying on the $C_{2}$ axis. This distance is consistent with a model for the $\mathrm{PtH}_{2}$ defect, in which the $\mathrm{H}$ atoms lie at anti-bonding sites $(A B)$, being back-bonded to two of the four Si neighbours of $\mathrm{Pt}^{-}$, as shown in Fig. 12. This model was first suggested by theoretical studies on $\mathrm{NiH}_{2}^{-}$[223]. Subsequent theoretical investigations on $\mathrm{PtH}_{2}^{-}[30]$ has supported this picture and found a Pt-H distance equal to $4.45 \AA$. A similar calculation for $\mathrm{AuH}_{2}^{0}$, determined this length to be $4.62 \AA[279]$.

Neutral $\mathrm{AuH}_{2}$ defects have also been detected and studied by IR absorption [267]. The vibrational spectra (Table 15) show similar features to those of the isoelectronic $\mathrm{PtH}_{2}^{-}$defect, suggesting an identical structure. Theory also finds close similarities between $\mathrm{AuH}_{2}$ and $\mathrm{PtH}_{2}$, as well as $\mathrm{AgH}_{2}$ and $\mathrm{PdH}_{2}$ defects [30, 228].

Several LVMs have been identified as arising from different charge states of trigonal or 
Table 14. Calculated [30, 228] and observed [267, 274] hydrogen and deuterium stretch frequencies $\left(\mathrm{cm}^{-1}\right)$ for $C_{2 v} \mathrm{PtH}_{2}$ complexes in three charge states, $q$. The observed values are assumed to correspond to the given charge states.

\begin{tabular}{ccccccccc}
\hline \hline & & \multicolumn{2}{c}{$q=0$} & \multicolumn{2}{c}{$q=-1$} & \multicolumn{2}{c}{$q=-2$} \\
& & Symm. & Calc. & Obs. & Calc. & Obs. & Calc. & Obs. \\
\hline \multirow{2}{*}{$\mathrm{PtH}_{2}$} & $A_{1}$ & 2081.4 & 1891.9 & 1986.2 & 1901.9 & 1959.8 & 1898.0 \\
& $B_{1}$ & 2077.5 & 1873.1 & 1986.1 & 1888.2 & 1943.5 & 1889.0 \\
& $A_{2}$ & 2079.5 & 1880.3 & 1986.2 & 1894.6 & 1951.7 & 1893.9 \\
& $B_{2}$ & 1495.2 & 1361.0 & 1429.2 & 1366.9 & 1400.9 & 1367.5 \\
& $A_{1}$ & 1496.6 & 1365.2 & 1429.2 & 1370.7 & 1406.9 & 1363.3 \\
& $B_{1}$ & 1493.8 & 1352.4 & 1429.1 & 1362.5 & 1395.1 & $\ldots$ \\
\hline \hline
\end{tabular}

near-trigonal $\mathrm{PtH}_{1}$ and $\mathrm{AuH}_{1}$ defects [274]. Their frequencies are very similar [267, 269] to each other (see Table 16), implying an identical structure. These are important observations since they demonstrate that the defect possesses several gap levels. In the case of $\mathrm{AuH}_{1}$, only three charge states have been detected. This is one less than found by the theory and DLTS studies (Section 9.5). For $\mathrm{PtH}_{1}$, two vibrational modes have been found corresponding to two different charge states.

Table 15. Calculated [30] and observed [267] hydrogen and deuterium stretch frequencies $\left(\mathrm{cm}^{-1}\right)$ for neutral $C_{2 v} \mathrm{AuH}_{2}$ complex. The observed values are assumed to correspond to the given charge states.

\begin{tabular}{cccc}
\hline \hline & Symm. & Calc. & Obs. \\
\hline \multirow{2}{*}{$\mathrm{AuH} \mathrm{H}_{2}$} & $A_{1}$ & 1947.1 & 1803.3 \\
& $B_{1}$ & 1970.5 & 1785.6 \\
$\mathrm{AuHD}$ & $A_{2}$ & 1972.6 & 1792.5 \\
& $B_{2}$ & 1419.2 & 1298.6 \\
$\mathrm{AuD}$ & $A_{1}$ & 1420.6 & 1304.4 \\
& $B_{1}$ & 1417.8 & 1292.1 \\
\hline \hline
\end{tabular}

Finally, we note that if $\mathrm{H}$ were bonded to $\mathrm{Si}$, then satellite lines due to ${ }^{29} \mathrm{Si}$ and ${ }^{30} \mathrm{Si}$ would be expected. The absence of these lines, together with the ease of the reorientation of the defect at cryogenic temperatures suggests that $\mathrm{H}$ is bonded directly to the TM impurity [269], contrary to the results of total energy calculations. This requires further investigation. 
Table 16. Comparison between the calculated [30, 228] and observed [267, 274] hydrogen and deuterium stretch frequencies $\left(\mathrm{cm}^{-1}\right)$ of $\mathrm{PtH}_{1}$ and $\mathrm{AuH}_{1}$ in charge state $q$. The observed values are assumed to correspond to the given charge states.

\begin{tabular}{lcccccc}
\hline \hline & \multicolumn{2}{c}{$q=0$} & \multicolumn{2}{c}{$q=-1$} & \multicolumn{2}{c}{$q=-2$} \\
\cline { 4 - 5 } & Calc. & Obs. & Calc. & Obs. & Calc. & Obs. \\
\hline $\mathrm{PtH}_{1}$ & 2034.1 & 1880.7 & 2022.2 & 1897.2 & $\ldots$ & $\ldots$ \\
$\mathrm{PtD}_{1}$ & 1464.3 & 1358.5 & 1453.2 & 1368.8 & $\ldots$ & $\ldots$ \\
$\mathrm{AuH}_{1}$ & 1947.4 & 1787.7 & 1890.5 & 1813.3 & 1893.2 & 1827.1 \\
$\mathrm{AuD}$ & 1401.1 & 1292.9 & 1359.6 & 1310.9 & 1361.4 & 1319.4 \\
\hline \hline
\end{tabular}

\subsection{Electrical levels of TM-H defects}

$\mathbf{P t}-\mathbf{H}$ and $\mathbf{P d}-\mathbf{H}$ defects A partial passivation of $\mathrm{Pt}$ by hydrogen was first observed by Pearton and Haller [176] in $n$-Si. However, the concentration of the $\operatorname{Pt}(0 /+)$ level detected in p-Si was unaffected by hydrogenation carried out using a H-plasma at $300^{\circ} \mathrm{C}$. Surprisingly, in view of later studies, no H-related levels were reported by these authors. Uftring and coworkers [274], as described above were able to locate approximately the electrical levels of $\mathrm{PtH}_{2}$, which is stable up to $600 \mathrm{~K}$, by varying the Fermi level position through electron irradiation. The $(-/ 0)$ level was placed between the $(-/ 0)$ and $(0 /+)$ levels of Pt, i.e. between $E_{c}-0.23 \mathrm{eV}$ and $E_{c}-0.9 \mathrm{eV}$, and the $(=/-)$ level between the $(0 /+)$ level of $\mathrm{P}$ at $E_{c}-0.045 \mathrm{eV}$ and the $(-/ 0)$ level of $\mathrm{C}_{i}$ at $E_{c}-0.1 \mathrm{eV}$. More recent work has pushed this level downwards to lie around $E_{c}-0.15 \mathrm{eV}[262]$. As discussed in Section 9.5, the FTIR absorption studies indicate that $\mathrm{PtH}_{1}$ has two charge states.

Studies using a wet chemical etch to introduce $\mathrm{H}$ revealed a plethora of $\mathrm{Pt}$-hydrogen levels. The DLTS spectrum of $n$-type hydrogenated Si samples doped with Pt exhibits two distinct peaks, $\mathrm{E}(90)$ and $\mathrm{E}(250)$, at $E_{c}-0.18$ and $E_{c}-0.50 \mathrm{eV}$, respectively [165, 178]. The smaller cross-section of the first is consistent with a $(=/-)$ level. Depth profiling and thermal annealing imply that $\mathrm{E}(90)$ and $\mathrm{E}(250)$ are levels of two different Pt-hydrogen defects which are stable up to $600 \mathrm{~K}$.

In a subsequent paper, Sachse et al. [165] reported two Pt-H related DLTS signals in $p$-type Si. The concentration of these $\mathrm{H}(150)$ and $\mathrm{H}(210)$ levels was shown to be strongly dependent upon the temperature and duration of the annealing procedure and that they arise from different 
defects. $\mathrm{H}(210)$ at $E_{v}+0.4 \mathrm{eV}$ is detected immediately after etching suggesting a defect with few hydrogen atoms. Its concentration increases after an anneal at $400 \mathrm{~K}$. Longer annealing times at $T=490 \mathrm{~K}$ revealed another peak, $\mathrm{H}(150)$, at $E_{v}+0.30 \mathrm{eV}$. The observation of a Poole-Frenkel effect suggests that $\mathrm{H}(150)$ is an acceptor $(-/ 0)$ level in the lower half of the band gap. Both $\mathrm{H}(150)$ and $\mathrm{H}(210)$ disappear after anneals above $T>650 \mathrm{~K}$. MCTS measurements [178] on p-type samples suggest that the concentration of $\mathrm{E}(250)$ is higher than that of $\mathrm{H}(210)$, implying that there are more $\mathrm{H}$ atoms in $\mathrm{H}(210)$.

Analysis of the depth profiles using Yarykin's procedure discussed in Section 9.4 gives $L_{n}$ for the $\mathrm{E}(90)$ and $\mathrm{E}(250)$ traps to be 0.80 and $1.55 \mu \mathrm{m}$ respectively [280]. This suggests that $\mathrm{E}(90)$ involves twice as many $\mathrm{H}$ atoms as E(250). Assuming that the complex responsible for E(250) contains a single $\mathrm{H}$ atom, then $\mathrm{E}(90)$ should be assigned to a $\mathrm{PtH}_{2}$ complex. The depth profiles also suggest that $\mathrm{E}(90)$ and $\mathrm{H}(210)$ arise from the same defect. It is now clear that $\mathrm{E}(90)$ is the $(=/-)$ level of $\mathrm{PtH}_{2}$. Consequently, $\mathrm{H}(210)$ is assigned the $(-/ 0)$ level of $\mathrm{PtH}_{2}$. This is consistent with EPR data showing that $\mathrm{PtH}_{2}$ has a deep $(-/ 0)$ level between $E_{c}-0.23 \mathrm{eV}$ and $E_{c}-0.9 \mathrm{eV}$. In this way Sachse et al. were able to assign DLTS lines to specific H defects. Their results are given in Table 13 along with the theoretical results deduced by cluster calculations described in Section 9.3.

The assignments were made as follows:

1. The calculations show that not only does $\mathrm{PtH}_{1}$ possess a $(-/ 0)$ level at $E_{c}-0.51 \mathrm{eV}$ in good agreement with the $0.50 \mathrm{eV}$ E(250) level, but also a deep donor level at $E_{v}+0.44 \mathrm{eV}$. There is no evidence for this level which might be obscured by the Pt $(0 /+)$ level at $E_{v}+0.30 \mathrm{eV}$. Alternatively, the calculated level may be in error by $\sim 0.2 \mathrm{eV}$ and the actual level falls below $E_{v}$. LDLTS studies should clarify these possibilities.

2. The $(=/-)$ level at $E_{c}-0.18 \mathrm{eV}$ due to $\mathrm{PtH}_{2}$ is used as a standard by the theoretical calculations. $\mathrm{H}(210)$ at $E_{c}-0.76 \mathrm{eV}$ is now assigned to the $(-/ 0)$ level. The calculated level at $E_{c}-0.47 \mathrm{eV}$ is about $0.3 \mathrm{eV}$ too shallow and this discrepancy originally suggested to us that $\mathrm{H}(210)$ was the $(0 /+)$ level of $\mathrm{PtH}_{1}[281]$. There should be an associated $(=/-)$ level but the lack of any firm experimental evidence for the $(=/-)$ level of $\mathrm{PtH}_{1}$, appears to rule this out [282]. Nevertheless, there should be a Poole-Frenkel effect associated with $\mathrm{H}(210)$ which has not, as far as we are aware, been reported. There is no donor level 
associated with $\mathrm{PtH}_{2}$.

3. The theoretical results show clearly that $\mathrm{PtH}_{3}$ has a deep acceptor level in the lower half of the band gap in agreement with the depth and character of $\mathrm{H}(150)$. Thus the assignment of this defect agrees with the conclusions of the DLTS profiling studies [280].

The DLTS studies have also provided evidence for the passivation of $\mathrm{Pt}$ in $n$-type samples. Near the sample surface, the total concentration of the three levels, E(90), $\mathrm{Pt}(-/ 0)$ and $\mathrm{E}(250)$, is less than that of $\mathrm{Pt}(-/ 0)$ in 'H-free' (cleaved) samples. This suggests that a passive defect has been formed, in the H-rich near-surface region, as a result of the interaction of atomic hydrogen with the Pt ion. The theory however gives no support that a substitutional Pt-H defect is passive.

DLTS studies on $\mathrm{Pd}-\mathrm{H}$ defects show close similarities with a $\mathrm{Pt}-\mathrm{H}$ ones enabling a similar set of assignments [234]. The calculations also show a close connection between corresponding defects and hence corresponding assignments can be made and are given in Table 13.

AuH and AgH defects Annealing and defect profiling studies have shown that the G1 (at $\left.E_{c}-0.19 \mathrm{eV}\right), \mathrm{G} 4$ and G2 levels due to gold-hydrogen defects arise from the same complex [168]. The location of the levels (see Table 13) and their emission cross-sections suggest that they correspond to $(=/-),(-/ 0)$ and $(0 /+)$ respectively. G2 was characterised as a donor level located at $E_{v}+0.21 \mathrm{eV}$ and this has been supported by MCTS measurements [269]. The deep penetration of these defects is taken to imply that they possess only one $\mathrm{H}$ atom.

Table 13 shows the observed G3 level of the $\mathrm{AuH}_{2}$ defect is within $0.2 \mathrm{eV}$ of the calculated donor level of $\mathrm{AuH}_{2}$. The profiling theory due to Yarykin et al. [167] also suggests that these levels should be assigned to $\mathrm{AuH}_{2}$. They are considered to be $(0 /+)$ levels [167].

The calculations place the $(-/ 0)$ level of $\mathrm{AuH}_{2}$ to be very close to that of $\mathrm{AuH}_{1}$. Evidence for the $(-/ 0)$ levels of $\mathrm{AuH}_{2}$ has been more difficult to obtain. However, recent Laplace DLTS studies has been able to decompose G4 into three separate defects with close-by levels $[172,173]$. These were the $(-/ 0)$ level of $\mathrm{Au}_{s}$ at $E_{c}-0.56 \mathrm{eV}$, the $\mathrm{G} 4(-/ 0)$ level due to $\mathrm{AuH}_{1}$ at $E_{c}-$ $0.54 \mathrm{eV}$, and the $\mathrm{G} 4^{\prime}$ level due to $\mathrm{AuH}_{2}$ at $E_{c}-0.58 \mathrm{eV}$. The assignment of $\mathrm{G}^{\prime}$ to the $(-/ 0)$ level of $\mathrm{AuH}_{2}$ along with assignments for all the other levels of $\mathrm{AuH}_{n}$ with $n<2$ is entirely in agreement with the theoretical calculations. Similar considerations apply to $\mathrm{Ag}-\mathrm{H}_{n}$ defects. 
We turn now to the levels of $\mathrm{AuH}_{3}$ and $\mathrm{AgH}_{3}$. The calculated (-/0) levels of $\mathrm{AgH}_{3}$ and $\mathrm{AuH}_{3}$ are relatively shallow. It may be that, with errors of order $0.2 \mathrm{eV}$, these defects are passive. This would explain the experimental observation that $\mathrm{H}$ can eliminate the electrical properties of $\mathrm{Au}$ and $\mathrm{Ag}$. However, in the case of $\mathrm{Au}$, a recent LDLTS finding [173] is that after a long room temperature anneal, a new level appeared at $E_{c}-0.13 \mathrm{eV}$. This is close to the calculated $(-/ 0)$ level of $\mathrm{AuH}_{3}$ although there is no evidence that the defect contains three $\mathrm{H}$ atoms. If this assignment is correct then the passive defect is unlikely to involve a substitutional TM atom.

Despite the obvious similarities between the structural and electrical properties of multihydrogen-Au and Ag defects, the differences in thermal stability between these remain to be explained. The $\mathrm{Ag}-\mathrm{H}$ complexes more stable than the $\mathrm{Au}$ analogue ones, annealing out at around $400-500{ }^{\circ} \mathrm{C}[167]$, vs. $250-300{ }^{\circ} \mathrm{C}$ for the $\mathrm{Au}-\mathrm{H}$ complexes [168].

\subsection{Electrical passivation}

As described previously, it is now clear that the electrical activity of substitutional Pt and $\mathrm{Pd}$ defects cannot be eliminated by the simple interaction with atomic hydrogen. However, calculations [30, 228] have shown that molecular hydrides $\mathrm{PtH}_{2}, \mathrm{PdH}_{2}$ and even $\mathrm{AuH}_{1}$ and $\mathrm{AgH}_{1}$ are all passive when inserted into lattice micro-voids induced by hydrogen. This suggests that passivation is not a simple process involving the bonding of $\mathrm{H}$ atoms to the TM impurity. Transition-metal ions have large atomic radius and are readily attracted to voids in the crystal through strain effects. This characteristic has been used to improve gettering [196, 197, 283].

This model for the electrical passivation of $\mathrm{Pt}$ and $\mathrm{Pd}$ could explain the results of Pearton and Haller [176]. It is known that the hydrogenation process used by these workers is quite aggressive, resulting in the creation of aggregates of vacancies in the near-surface region of the samples. However, it is not clear whether chemical etching, results in the formation of aggregates of vacancies yet this is clearly a requirement of the model.

\section{Concluding Remarks}

It is clear that our understanding of complexes of hydrogen with deep level defects is in its infancy. The particular defects introduced by hydrogenation depend upon the technique used. 
Low temperature proton implantation leads to isolated primitive defects and perhaps offers the best way forward in understanding their evolution with temperature. Interpretation will, however, be complicated by the generation of metastable defects like $\mathrm{H}_{2}^{*}$. These defects are not created by high temperature in-diffusion which succeeds largely in creating hydrogen molecules along with impurity - hydrogen defects such as $\mathrm{B}-\mathrm{H}, \mathrm{PtH}_{n}$ and $\mathrm{AuH}_{n}$. The most common technique used, plasma treatments, produce a much richer spectrum of defects including extended ones, such as platelets.

From a theoretical viewpoint, the behaviour of isolated $\mathrm{H}$ is understood at least on a semiquantitative footing. The interaction of $\mathrm{H}$ with shallow dopants is largely resolved in $\mathrm{Si}-$ although there are fundamental problems in other materials such as Ge and diamond — but the interaction of hydrogen with neutral defects like carbon and oxygen is still an active area. There are continuing uncertainties with the way $\mathrm{H}$ defects evolve in various heat treatments. For example, in implanted material the formation mechanisms of $\mathrm{H}_{2}^{*}, \mathrm{VH}_{n}$ and $\mathrm{IH}_{2}$ can only be guessed.

The structure of the hydrogen molecule within Si is not completely understood. There are uncertainties about, for example, their alignment within the lattice, or whether the infra-red active species are complexes with other impurities or defects. There are unanswered questions about the effects of $e$-irradiation on Si containing these molecules. The observation of only one defect namely $\mathrm{H}_{2}^{*}$ and the absence of complexes with vacancies is both surprising and unexpected and in contrast with results on $e$-irradiation of plasma treated material which leads to a great number of $\mathrm{V}_{n} \mathrm{H}_{m}$ defects.

Surprisingly, the elucidation of the properties of transition metal hydrogen defects has been rapid: the electrical properties of substitutional Au and Pt when complexed with hydrogen are now reasonably established. One of the main effects of $\mathrm{H}$ is to lower the position of the donor levels which disappear into the valence band after one or two $\mathrm{H}$ atoms have been added. This is a consequence of the Coulomb field arising from the protons. In some cases, new levels are introduced, for example the $(=/-)$ levels of $\mathrm{AuH}_{1}$ and $\mathrm{PtH}_{2}$. In others, there is only a minute shift in the levels of the isolated impurity, e.g., the $(-/ 0)$ levels of $\mathrm{AuH}_{1}$ and $\mathrm{AuH}_{2}$. A key role for $\mathrm{H}$ is in the addition of electrons to the $t_{2}$-manifold and when this becomes filled, there is a possibility of elimination of the electrical activity if higher levels arising from $5 s$ and $6 s$ orbitals 
do not enter the band gap. The theory, which also is in its infancy for these systems, suggests that substitutional Pt and Pd defects cannot be passivated but this is less clear in the case of $\mathrm{Ag}$ and $\mathrm{Au}$.

It is clear that the interaction between hydrogen and defects with deep levels is an area of growing importance because of the implications for semiconductor devices. However, there is a need for more sophisticated experiments and theories than are currently available.

\section{Acknowledgements}

The authors thank Michael Budde, Brian Bech Nielsen, Michael Stavola and Ron Newman for many useful discussions.

\section{References}

[1] S. M. Myers, M. I. Baskes, H. K. Birnbaum, J. W. Corbett, G. G. DeLeo, S. K. Estreicher, E. E. Haller, P. Jena, N. M. Johnson, R. Kirchheim, S. J. Pearton, and M. J. Stavola, Rev. Mod. Phys. 64, 559 (1992).

[2] S. K. Estreicher, Mater. Sci. Eng. R 14, 319 (1995).

[3] C. G. Van de Walle, Journal of Vacuum Sci. and Technol. A 16, 1767 (1998).

[4] Theory of the inhomogeneous electron gas, edited by S. Lundqvist and N. H. March (Plenum, New York, 1983).

[5] J. Ihm, Rep. Prog. Phys. 51, 105 (1988).

[6] J. A. Pople, J. S. Binkley, and R. Seeger, Int. J. Quant. Chem. Symp. 10, 1 (1976).

[7] M. T. Yin and M. L. Cohen, Phys. Rev. B 25, 7403 (1982).

[8] G. B. Bachelet, D. R. Hamann, and M. Schlüter, Phys. Rev. B 26, 4199 (1982).

[9] G. B. Bachelet and M. Schlüter, Phys. Rev. B 25, 2103 (1982).

[10] P. Deák and L. C. Snyder, International journal of quantum chemistry S21, 547 (1987).

[11] T. A. Halgren and W. N. Lipscomb, J. Chem. Phys. 58, 1569 (1973).

[12] M. Saito and A. Oshiyama, Phys. Rev. B 38, 10711 (1988).

[13] R. Jones and P. R. Briddon, in Identification of Defects in Semiconductors, Vol. 51A of Semiconductors and Semimetals, edited by M. Stavola (Academic Press, Boston, 1998), Chap. 6, p. 287.

[14] P. J. Kelly and R. Car, Phys. Rev. B 45, 6543 (1992).

[15] C. G. Van de Walle, Y. Bar-Yam, and S. T. Pantelides, Phys. Rev. Lett. 60, 2761 (1988).

[16] K. J. Chang and D. J. Chadi, Phys. Rev. B 40, 11644 (1989).

[17] M. Car and M. Parrinello, Phys. Rev. Lett. 55, 2471 (1985). 
[18] R. B. Capaz, L. V. C. Assali, L. C. Kimerling, K. Cho, and J. D. Joannopoulos, Phys. Rev. B 59, 4898 (1999).

[19] T. L. Estle, S. K. Estreicher, and D. S. Marynick, Hyperfine Interactions 32, 637 (1986).

[20] S. Estreicher, Phys. Rev. B 36, 9122 (1987).

[21] C. H. Chu and S. K. Estreicher, Phys. Rev. B 42, 9486 (1990).

[22] P. Deák, L. C. Snyder, and J. W. Corbett, Phys. Rev. B 37, 6887 (1988).

[23] P. Deák, L. C. Snyder, J. L. Lindstrom, J. W. Corbett, S. J. Pearton, and A. J. Tavendale, Phys. Lett. A 126, 427 (1988).

[24] P. Deák, L. C. Snyder, and J. W. Corbett, Phys. Rev. B 43, 4545 (1991).

[25] C. G. Van de Walle, P. J. H. Denteneer, Y. Bar-Yam, and S. T. Pantelides, Phys. Rev. B 39, 10791 (1989).

[26] G. G. DeLeo, M. J. Dorogi, and W. B. Fowler, Phys. Rev. B 38, 7520 (1988).

[27] R. Jones, Physica B 170, 181 (1991).

[28] P. R. Briddon and R. Jones, Hyperfine Interactions 64, 593 (1990).

[29] N. M. Johnson, C. Herring, and C. G. Van de Walle, Phys. Rev. Lett. 73, 130 (1994).

[30] A. Resende, R. Jones, P. R. Briddon, and S. Öberg, Phys. Rev. Lett. 82, 2111 (1999).

[31] C. G. Van de Walle, Phys. Rev. B 49, 4579 (1994).

[32] P. Deák, M. Heinrich, L. C. Snyder, and J. W. Corbett, Mater. Sci. Eng. B 4, 57 (1989).

[33] P. Deák, L. C. Snyder, M. Heinrich, C. R. Ortiz, and J. W. Corbett, Physica B 170, 253 (1991).

[34] M. Budde, Ph.D. thesis, Aarhus Center for Atomic Physics, University of Aarhus, Denmark, 1998.

[35] Y. V. Gorelkinskii and N. N. Nevinnyi, Physica B 170, 155 (1991).

[36] B. Bech Nielsen, Phys. Rev. B 37, 6353 (1988).

[37] K. Irmscher, K. Klose, and K. Maass, J. Phys. C 17, 6317 (1984).

[38] B. Holm, K. Bonde Nielsen, and B. Bech Nielsen, Phys. Rev. Lett. 66, 2360 (1991).

[39] C. H. Seager, R. A. Anderson, and S. K. Estreicher, Phys. Rev. Lett. 74, 4565 (1995).

[40] N. M. Johnson, C. Herring, and C. G. Van de Walle, Phys. Rev. Lett. 74, 4566 (1995).

[41] S. F. J. Cox and M. C. R. Symmons, Chem. Phys. Lett. 126, 516 (1986).

[42] T. L. Estle, S. Estreicher, and D. S. Marynick, Phys. Rev. Lett. 58, 1547 (1987).

[43] J. H. Brewer, K. M. Crowe, F. N. Gygax, R. F. Johnson, R. F. Patterson, D. G. Fleming, and A. Schenk, Phys. Rev. Lett. 31, 143 (1973).

[44] C. G. Van de Walle and P. E. Blöchl, Phys. Rev. B 47, 4244 (1993).

[45] B. Bech Nielsen, K. Bonde Nielsen, and J. R. Byberg, Mater. Sci. Forum 143-147, 909 (1994).

[46] Y. V. Gorkelkinskii and N. N. Nevinnyi, Mater. Sci. Eng. B 36, 133 (1996). 
[47] A. Van Weiringen and N. Warmholz, Physica 22, 849 (1956).

[48] H. K. Stein, Phys. Rev. Lett. 43, 1030 (1979).

[49] B. Hitti, S. R. Kreitzman, T. L. Estle, E. S. Bates, M. R. Dawdy, T. L. Head, and R. L. Litchi, Phys. Rev. B 59, 4918 (1999).

[50] P. R. Briddon, R. Jones, and G. M. S. Lister, J. Phys. C 21, L1027 (1988).

[51] K. J. Chang and D. J. Chadi, Phys. Rev. Lett. 62, 937 (1989).

[52] M. Budde, B. Bech Nielsen, R. Jones, J. Goss, and S. Öberg, Phys. Rev. B 54, 5485 (1996).

[53] J. D. Holbech, B. Bech Nielsen, R. Jones, P. Sitch, and S. Öberg, Phys. Rev. Lett. 71, 875 (1993).

[54] B. N. Mukashev, M. F. Tamendarov, and S. Z. Tokmoldin, Sov. Phys. Semicond. 26, 628 (1992).

[55] S. B. Zhang, W. B. Jackson, and D. J. Chadi, Phys. Rev. Lett. 65, 2575 (1990).

[56] K. J. Chang and D. J. Chadi, Phys. Rev. B 42, 7651 (1990).

[57] G. D. Watkins and J. W. Corbett, Phys. Rev. 138, A543 (1965).

[58] Y. H. Lee and J. W. Corbett, Phys. Rev. B 8, 2180 (1973).

[59] Y. H. Lee and J. W. Corbett, Phys. Rev. B 9, 4351 (1974).

[60] S. K. Estreicher, J. L. Hastings, and P. A. Fedders, Appl. Phys. Lett. 70, 432 (1997).

[61] M. A. Roberson and S. K. Estreicher, Phys. Rev. B 49, 17040 (1994).

[62] B. Bech Nielsen, L. Hoffman, and M. Budde, Mater. Sci. Eng. B 36, 259 (1996).

[63] A. N. Nazarov, V. M. Pinchuk, and T. V. Yanchuk, Model. Simul. Mater. Sci. Eng. 4, 323 (1996).

[64] H. Xu, Phys. Rev. B 46, 1403 (1992).

[65] S. K. Estreicher, J. L. hastings, and P. A. Fedders, in Materials Science Forum (Trans Tech Publications, Switzerland, in press).

[66] Y. K. Park, S. K. Estreicher, and C. W. Myles, Phys. Rev. B 52, 1718 (1995).

[67] B. Bech Nielsen, L. Hoffman, M. Budde, R. Jones, J. Goss, and S. Öberg, Mater. Sci. Forum 196-201, 933 (1995).

[68] L. M. Xie, M. W. Qi, and J. M. Chen, J. Phys. Cond. Matter 3, 8519 (1991).

[69] W. M. Chen, O. O. Awadelkarim, and B. Monemar, Phys. Rev. Lett. 64, 3042 (1990).

[70] B. Bech Nielsen, P. Johannesen, P. Stallinga, and K. Bonde Nielsen, Phys. Rev. Lett. 79, 1507 (1997).

[71] P. Stallinga, P. Johannesen, S. Herstrom, K. Bond Nielsen, B. Bech Nielsen, and J. R. Byberg, Phys. Rev. B 58, 3842 (1998).

[72] S. K. Estreicher, J. L. Hastings, and P. A. Fedders, Phys. Rev. B 57, 12663 (1998).

[73] B. Hourahine, B. J. Coomer, R. Jones, and S. Öberg, (unpubished).

[74] M. Suezawa, Japan J. Appl. Phys. 37, L806 (1998). 
[75] M. Suezawa, J. Appl. Phys. 83, 1958 (1998).

[76] B. Hourahine, R. Jones, S. Öberg, and P. R. Briddon, (to appear in Phys. Rev. B).

[77] B. Bech Nielsen, L. Hoffman, M. Budde, R. Jones, J. Goss, and S. Öberg, in Defects in Semiconductors 18, edited by M. Suezawa and H. Katyama-Yoshida (Trans Tech, Aedermannsdork, 1995), Vol. 196-201, p. 933.

[78] S. Herstrøm, Master's thesis, Institute of Physics and Astronomy, University of Aarhus, 1998, to be published.

[79] S. K. Estreicher, Mater. Sci. Eng. R 14, 317 (1997).

[80] R. Jones, J. Goss, C. Ewels, and S. Öberg, Phys. Rev. B 50, 8378 (1994).

[81] A. Resende, J. P. Goss, R. Jones, S. Öberg, and P. R. Briddon, (to be published).

[82] S. J. Pearton, J. W. Corbett, and T. S. Shi, Appl. Phys. A 43, 153 (1987).

[83] B. J. Coomer, P. Leary, M. Budde, B. Bech Nielsen, R. Jones, S. Öberg, and P. R. Briddon, Mater. Sci. Eng. B (in press).

[84] E. E. Haller, G. S. Hubbard, W. L. Hansen, and A. Seeger, Inst. Phys. Conf. Ser. 31, 309 (1977).

[85] M. Pesola, J. von Boehm, S. Pöykkö, and R. M. Nieminen, Phys. Rev. B 58, 1106 (1998).

[86] M. Saito and A. Oshiyama, Phys. Rev. Lett. 73, 866 (1994).

[87] H. Seong and L. J. Lewis, Phys. Rev. B 53, 9791 (1996).

[88] B. J. Coomer, A. Resende, and R. Jones, (to be published).

[89] C. G. Van de Walle and J. Neugebauer, Phys. Rev. B 52, 14320 (1995).

[90] M. Budde, B. Bech Nielsen, P. Leary, J. Goss, R. Jones, P. R. Briddon, S. Öberg, and S. J. Breuer, Mater. Sci. Forum 258-263, 35 (1997).

[91] M. Budde, B. Bech Nielsen, P. Leary, J. Goss, R. Jones, P. R. Briddon, S. Öberg, and S. J. Breuer, Phys. Rev. B 57, 4397 (1998).

[92] Y. Baryam and J. D. Joannopoulos, Phys. Rev. Lett. 52, 1129 (1984).

[93] R. Car, P. J. Kelly, A. Oshiyama, and S. T. Pantelides, Phys. Rev. Lett. 54, 360 (1985).

[94] P. E. Blöchl, E. Smargiassi, R. Car, D. B. Laks, W. Andreoni, and S. T. Pantelides, Phys. Rev. Lett. 70, 2435 (1993).

[95] D. J. Chadi, Phys. Rev. B 46, 9400 (1992).

[96] J. F. Zheng, M. Stavola, and G. D. Watkins, in The Physics of Semiconductors, edited by D. J. Lockwood (World Scientific, Singapore, 1994), p. 2363.

[97] P. Leary, R. Jones, S. Öberg, and V. J. B. Torres, Phys. Rev. B 55, 2188 (1997).

[98] B. Bech Nielsen, L. Hoffmann, M. Budde, R. Jones, J. Goss, and S. Öberg, Mater. Sci. Forum 196-201, 933 (1995).

[99] J. W. Corbett, S. N. Sahu, T. S. Shi, and L. C. Snyder, Phys. Lett. A 93, 303 (1983).

[100] A. Mainwood and A. M. Stoneham, Physica B\&C 116, 101 (1983).

[101] M. Stutzmann, W. Beyer, L. Tapfer, and C. P. Herrero, Physica B 170, 240 (1991). 
[102] J. B. Boyce, N. M. Johnson, S. E. Ready, and J. Walker, Phys. Rev. B 46, 4308 (1992).

[103] K. Murakami, N. Fukata, S. Sasaki, K. Ishioka, M. Kitajima, S. Fujimura, J. Kikuchi, and H. Haneda, Phys. Rev. Lett. 77, 3161 (1996).

[104] J. Vetterhöffer, J. Wagner, and J. Weber, Phys. Rev. Lett. 77, 5409 (1996).

[105] N. Fukata, K. Murakami, K. Ishioka, K. G. Nakamura, M. Kitajima, S. Fujimura, J. Kikuchi, and H. Haneda, Mater. Sci. Forum 258-263, 211 (1997).

[106] A. W. R. Leitch, V. Alex, and J. Weber, Phys. Rev. Lett. 81, 421 (1998).

[107] P. Stallinga, T. Gregorkiewicz, C. A. J. Ammerlaan, and Y. V. Gorkelkinskii, Mater. Sci. Forum 143-147, 853 (1994).

[108] M. J. Binns, R. C. Newman, S. A. McQuaid, and E. C. Lightowlers, Mater. Sci. Forum 143-147, 861 (1994).

[109] V. P. Markevich, M. Suezawa, and K. Sumino, Mater. Sci. Forum 196-201, 915 (1995).

[110] V. P. Markevich, I. F. Medvedeva, and L. I. Murin, in Early stages of oxygen precipitation in silicon, Vol. 17 of NATO ASI - 3. High Technology, edited by R. Jones (Kluwer Accademic Publishers, Dordrecht, 1996), p. 103.

[111] V. P. Markevich and M. Suezawa, J. Appl. Phys. 83, 2988 (1998).

[112] R. E. Pritchard, M. J. Ashwin, R. C. Newman, J. H. Tucker, E. C. Lightowlers, M. J. Binns, R. Falster, and S. A. Mcquaid, Mater. Sci. Forum 258-263, 283 (1997).

[113] R. E. Pritchard, M. J. Ashwin, J. H. Tucker, R. C. Newman, E. C. Lightowlers, M. J. Binns, S. A. Mcquaid, and R. Falster, Phys. Rev. B 56, 13118 (1997).

[114] R. E. Pritchard, M. J. Ashwin, J. H. Tucker, and R. C. Newman, Phys. Rev. B 57, 15048 (1998).

[115] A. W. R. Leitch, V. Alex, and J. Weber, Mater. Sci. Forum 258-263, 241 (1997).

[116] A. W. R. Leitch, V. Alex, and J. Weber, Solid State Commun. 105, 215 (1998).

[117] N. Fukata, S. Sasaki, K. Murakami, K. Ishioka, M. Kitajima, S. Fujimura, and J. Kikuchi, Jap. J. Appl. Phys. Part 2 35, L1069 (1996).

[118] M. Kitajima, K. Ishioka, K. G. Nakamura, N. Fukata, K. Murakami, J. Kikuchi, and S. Fujimura, Mater. Sci. Forum 258-263, 203 (1997).

[119] K. Ishioka, K. G. Nakamura, M. Kitajima, N. Fukata, K. Murakami, S. Fujimura, and J. Kikuchi, Mater. Sci. Forum 258-263, 235 (1997).

[120] K. Ishioka, K. G. Nakamura, M. Kitajima, N. Fukata, S. Sasaki, K. Murakami, S. Fujimura, J. Kikuchi, and H. Haneda, Appl. Surf. Sci. 117, 37 (1997).

[121] K. G. Nakamura, K. Ishioka, M. Kitajima, N. Fukata, K. Murakami, A. Endou, M. Kubo, and A. Miyamoto, Appl. Surf. Sci. 132, 243 (1998).

[122] N. Fukata, S. Sasaki, K. Murakami, K. Ishioka, K. G. Nakamura, M. Kitajima, S. Fujimura, J. Kikuchi, and H. Haneda, Phys. Rev. B 56, 6642 (1997).

[123] A. Mainwood and A. M. Stoneham, J. Phys. C 17, 2513 (1984).

[124] P. Deák and L. C. Snyder, Radiation Effects and Defects in Solids 111 \& 112, 77 (1989). 
[125] K. J. Chang and D. J. Chadi, Phys. Rev. Lett. 60, 1422 (1988).

[126] S. K. Estreicher, M. A. Roberson, and D. M. Maric, Phys. Rev. B 50, 17018 (1994).

[127] D. M. Maric, M. A. Roberson, and S. K. Estreicher, Mater. Sci. Forum 143-147, 1245 (1994).

[128] C. G. Van de Walle, Physica B 170, 21 (1991).

[129] C. G. Van de Walle, Phys. Rev. Lett. 80, 2177 (1998).

[130] C. G. Van de Walle, Phys. Rev. B 58, 1689 (1998).

[131] L. D. Landau and E. M. Lifshitz, Quantum mechanics, 3rd ed. (Pergamon, Oxford, 1977).

[132] Y. Okamoto, M. Saito, and A. Oshiyama, Phys. Rev. B 56, 10016 (1997).

[133] Y. Okamoto, M. Saito, and A. Oshiyama, Phys. Rev. B 58, 7701 (1998).

[134] K. G. Nakamura, K. Ishioka, M. Kitajima, A. Endou, M. Kubo, and A. Miyamoto, J. Chem. Phys. 108, 3222 (1998).

[135] K. G. Nakamura, K. Ishioka, M. Kitajima, and K. Murakami, Solid State Commun. 101, 735 (1997).

[136] K. G. Nakamura and M. Kitajima, Jap. J. Appl. Phys. Part 1 36, 2004 (1997).

[137] K. G. Nakamura, K. Ishioka, and M. Kitajima, Jap. J. Appl. Phys. Part 2 36, L1479 (1997).

[138] B. Hourahine, R. Jones, S. Öberg, R. C. Newman, P. R. Briddon, and E. Roduner, Phys. Rev. B 57, 12666 (1998).

[139] S. J. Breuer, R. Jones, P. R. Briddon, and S. Öberg, Phys. Rev. B 53, 16289 (1996).

[140] B. Hourahine, R. Jones, S. Öberg, and P. R. Briddon, Mater. Sci. Eng. B (in press).

[141] S. Muto, S. Takeda, and M. Hirata, Phil. Mag. A 72, 1057 (1995).

[142] P. Deák, C. R. Ortiz, L. C. Snyder, and J. W. Corbett, Physica B 170, 223 (1991).

[143] N. M. Johnson, F. A. Ponce, R. A. Street, and R. J. Nemanich, Phys. Rev. B 35, 4166 (1987).

[144] H. J. Stein, S. M. Myers, and D. M. Follstaedt, J. Appl. Phys. 73, 2755 (1993).

[145] Y. J. Chabal and C. K. N. Patel, Phys. Rev. Lett. 53, 1771 (1984).

[146] J. Weber, private communication, 1998.

[147] B. Hourahine, R. Jones, S. Öberg, and P. R. Briddon, Mater. Sci. Forum 258-263, 277 (1997).

[148] B. Bech Nielsen, K. Tanderup, M. Budde, K. Bond Nielsen, J. L. Lindstrom, R. Jones, S. Öberg, B. Hourahine, and P. R. Briddon, Mater. Sci. Forum 258-263, 391 (1997).

[149] R. C. Newman, J. H. Tucker, A. R. Brown, and S. A. McQuaid, J. Appl. Phys. 70, 3061 (1991).

[150] H. J. Stein and S. K. Hahn, J. Appl. Phys. 75, 3477 (1994).

[151] S. K. Estreicher, Phys. Rev. B 41, 9886 (1990).

[152] S. Fabian, S. Kalbitzer, C. Klatt, M. Behar, and C. Langpape, Phys. Rev. B 58, 16144 (1998).

[153] S. K. Estreicher, J. L. Hastings, and P. A. Fedders, Phys. Rev. Lett. 82, 815 (1999).

[154] S. Muto, S. Takeda, and M. Hirata, Mater. Sci. Forum 196-201, 1171 (1995). 
[155] L. Meda, G. F. Cerofolini, G. Ottaviani, T. R., F. Corni, R. Balboni, A. M., R. Canteri, and R. Dierckx, Physica B 170, 259 (1991).

[156] M. J. Binns, S. A. McQuaid, R. C. Newman, and E. C. Lightowlers, Semicond. Sci. Technol. 8, 1908 (1993).

[157] R. E. Pritchard, J. H. Tucker, R. C. Newman, and E. C. Lightowlers, Semicond. Sci. Technol. 14, 77 (1999).

[158] N. M. Johnson, C. Herring, C. Doland, G. Walker, G. Anderson, and F. A. Ponce, Mater. Sci. Forum 83-87, 33 (1992).

[159] N. M. Johnson, C. Doland, F. Ponce, J. Walker, and G. Anderson, Physica B 170, 3 (1991).

[160] S. B. Zhang and W. B. Jackson, Phys. Rev. B 43, 12142 (1991).

[161] A. J. Tavendale, A. A. Williams, and S. J. Pearton, Mater. Res. Soc. Symp. Proc 104, 285 (1988).

[162] M. Yoneta, Y. Kamimura, and F. Hashimoto, J. Appl. Phys. 70, 1295 (1991).

[163] Y. Tokuda, Japan J. Appl. Phys. 37, 1815 (1998).

[164] M. Shiraishi, J.-U. Sachse, H. Lemke, and J. Weber, Mater. Sci. Eng. B (in press).

[165] J.-U. Sachse, E. Ö. Sveinbjörnsson, W. Jost, and J. Weber, Phys. Rev. B 55, 16176 (1997).

[166] J.-U. Sachse, E. Ö. Sveinbjörnsson, N. Yarykin, and J. Weber, Mater. Sci. Eng. B (in press).

[167] N. Yarykin, J.-U. Sachse, H. Lemke, and J. Weber, Phys. Rev. B 59, 5551 (1999).

[168] E. Ö. Sveinbjörnsson and O. Engström, Phys. Rev. B 52, 4884 (1995).

[169] E. Ö. Sveinbjörnsson, G. I. Andersson, and O. Engström, Phys. Rev. B 49, 7801 (1994).

[170] M. Kouketsu, K. Watanabe, and S. Isomae, Mater. Sci. Forum 196-201, 861 (1995).

[171] J. A. Davidson and J. H. Evans, Semicond. Sci. Technol. 11, 1704 (1996).

[172] P. Deixler, J. Terry, I. D. Hawkins, J. H. Evans-Freeman, A. R. Peaker, L. Rubaldo, D. K. Maude, J.-C. Portal, L. Dobaczewski, K. Bonde Nielsen, and A. Nylandsted Larsen, Appl. Phys. Lett. 73, 3126 (1998).

[173] L. Rubaldo, P. Deixler, I. D. Hawkins, J. Terry, D. K. Maude, J.-C. Portal, J. H. Evans-Freeman, L. Dobaczewski, and A. R. Peaker, Mater. Sci. Eng. B (in press).

[174] T. Sadoh, K. Tsukamoto, A. Baba, D. Bai, A. Kenjo, and T. Tsurushima, J. Appl. Phys. 82, 3828 (1997).

[175] S. J. Pearton and A. J. Tavendale, Phys. Rev. B 26, 7105 (1982).

[176] S. J. Pearton, J. M. Kahn, W. L. Hansen, and E. E. Haller, J. Appl. Phys. 55, 1464 (1983).

[177] S. J. Pearton and A. J. Tavendale, J. Phys. C 17, 6701 (1984).

[178] J.-U. Sachse, E. Ö. Sveinbjörnsson, W. Jost, and J. Weber, Appl. Phys. Lett. 70, 1584 (1997).

[179] W. Stöffler and J. Weber, Phys. Rev. B 33, 8892 (1986).

[180] H. Lemke, Phys. Stat. Sol. A 101, 193 (1987).

[181] Y. K. Kwon, T. Ishikawa, and H. Kuwano, J. Appl. Phys. 61, 1055 (1987). 
[182] A. A. Gill, N. Baber, and M. Z. Iqbal, J. Appl. Phys. 67, 1130 (1990).

[183] M. Höhne, Phys. Rev. B 45, 5883 (1992).

[184] H. J. von Bardeleben, D. Stiévenard, M. Brousseau, and J. Barrau, Phys. Rev. B 38, 6308 (1988).

[185] M. Höhne and U. Juda, Mat. Sci. Technol. 11, 680 (1995).

[186] U. Juda, O. Scheerer, M. Höhne, H. Riemann, H.-J. Schilling, J. Donecker, and A. Gerhardt, J. Appl. Phys. 80, 3435 (1996).

[187] K. Graff, Metal Impurities in Silicon-Device Fabrication (Springer, Berlin, 1995).

[188] R. L. Kleinhenz, Y. H. Lee, J. W. Corbett, E. G. Sieverts, S. H. Müller, and C. A. J. Ammerlaan, Phys. Stat. Sol. A 108, 363 (1981).

[189] P. Alteheld, S. Greulich-Weber, J.-M. Spaeth, H. Overhof, and M. Höhne, Mater. Sci. Forum 143-147, 1173 (1994).

[190] A. Thilderkvist, S. G. Nilsson, M. Kleverman, and H. G. Grimmeiss, Phys. Rev. B 49, 16926 (1994).

[191] P. M. Williams, P. W. Mason, and G. D. Watkins, Phys. Rev. B 53, 12570 (1996).

[192] P. N. Hai, T. Gregorkiewicz, C. A. J. Ammerlaan, and D. T. Don, Mater. Sci. Forum 258-263, 491 (1997).

[193] J.-M. Spaeth, S. Martini, and S. Greulich-Weber, Semicond. Sci. Technol. 13, 725 (1998).

[194] W. Lerch, N. A. Stowijk, H. Meher, and C. Poisson, Semicond. Sci. Technol. 10, 1257 (1995).

[195] D. C. Schmidt, B. G. Svensson, N. Keskitalo, S. Godey, E. Ntsoenzok, J. Barbot, and C. Blanchard, J. Appl. Phys. 84, 4214 (1998).

[196] B. Mohadjeri, J. S. Williams, and J. Wong-Leung, Appl. Phys. Lett. 66, 1889 (1995).

[197] A. Kinomura, J. S. Williams, J. Wong-Leung, and M. Petravic, Appl. Phys. Lett. 72, 2713 (1998).

[198] V. C. Venezia, D. Englesham, T. E. Haynes, A. Agarwal, D. C. Jacobson, H.-J. Gossmann, and F. H. Baumann, Appl. Phys. Lett. 73, 2980 (1998).

[199] G. W. Ludwig and H. H. Woodbury, in Electron Spin Resonance in Semiconductors, Vol. 13 of Solid State Physics, edited by F. Seitz and D. Turnbull (Academic Press, New York, 1962).

[200] A. Thiderkvist, G. Grossmann, M. Kleverman, and H. G. Grimmeiss, Phys. Rev. B 58, 7723 (1998).

[201] J. W. Petersen and J. Nielsen, Appl. Phys. Lett. 56, 1122 (1990).

[202] G. D. Watkins, M. Kleverman, A. Thilderkvist, and H. G. Grimmeiss, Phys. Rev. Lett. 67, 1149 (1991).

[203] N. T. Són, V. E. Kustov, T. Gregorkiewicz, and C. A. J. Ammerlaan, Phys. Rev. B 46, 4544 (1992).

[204] H. H. Woodbury and G. W. Ludwig, Phys. Rev. 126, 466 (1962).

[205] F. G. Anderson, F. S. Ham, and G. D. Watkins, Phys. Rev. B 45, 3287 (1992).

[206] F. G. Anderson, R. F. Milligan, and G. D. Watkins, Phys. Rev. B 45, 3279 (1992). 
[207] L. S. Vlasenko, N. T. Són, A. B. von Oosten, C. A. J. Ammerlaan, A. A. Lebedev, E. S. Tapygov, and V. A. Khramtsov, Solid State Commun. 73, 393 (1990).

[208] N. T. Són, A. B. von Oosten, and C. A. J. Ammerlaan, Solid State Commun. 80, 439 (1990).

[209] U. Gösele, W. Frank, and A. Seeger, Appl. Phys. 23, 361 (1980).

[210] S. Mantovani, F. Nava, C. Nobili, and G. Ottaviani, Phys. Rev. B 33, 5536 (1986).

[211] A. B. van Oosten, N. T. Són, L. S. Vlasenko, and C. A. J. Ammerlaan, Mater. Sci. Forum 38-41, 355 (1989).

[212] N. T. Són, A. B. von Oosten, and C. A. J. Ammerlaan, Solid State Commun. 80, 439 (1991).

[213] G. D. Watkins and P. M. Williams, Phys. Rev. B 52, 16575 (1995).

[214] R. F. Milligan, F. G. Anderson, and G. D. Watkins, Phys. Rev. B 29, 2819 (1984).

[215] F. G. Anderson, C. Delerue, M. Lannoo, and G. Allan, Phys. Rev. B 44, 10925 (1991).

[216] G. D. Watkins, Physica B\&C 117-118, 9 (1983).

[217] L. A. Hemstreet, Phys. Rev. B 15, 834 (1976).

[218] B. G. Cartling, J. Phys. C 8, 3183 (1975).

[219] J. L. A. Alves and J. R. Leite, Phys. Rev. B 34, 7174 (1986).

[220] G. D. Watkins, in Defects and Their Structure in Non-metallic Solids, edited by B. Henderson and A. E. Hughes (Plenum, London, 1976).

[221] G. D. Watkins, in Deep Centers in Semiconductors, 2nd ed., edited by S. T. Pantelides (Gordon and Breach, Switzerland, 1992), Chap. 3.

[222] P. M. Williams, G. D. Watkins, S. Uftring, and M. Stavola, Mater. Sci. Forum 143-147, 891 (1994).

[223] R. Jones, S. Öberg, J. Goss, P. R. Briddon, and A. Resende, Phys. Rev. Lett. 75, 2734 (1995).

[224] G. W. Ludwig and H. H. Woodbury, Phys. Rev. Lett. 5, 98 (1960).

[225] J. Isoya, H. Kanda, J. R. Norris, J. Tang, and M. K. Bowman, Phys. Rev. B 41, 3905 (1990).

[226] J. Goss, A. Resende, R. Jones, S. Öberg, and P. R. Briddon, Mater. Sci. Forum 196-201, 67 (1995).

[227] M. Kleverman, A. Thilderkvist, G. Grossman, H. G. Grimmeiss, and G. D. Watkins, Solid State Commun. 93, 383 (1995).

[228] A. Resende, R. Jones, S. Öberg, and P. R. Briddon, (to be published).

[229] M. H. Woodbury and W. W. Tyler, Phys. Rev. 105, 2080 (1957).

[230] H. Lemke, in Semiconductor Silicon, edited by H. R. Huff, W. Bergholz, and K. Sumino (The Electrochemical Society, Pennington, NJ, 1994), p. 695.

[231] A. Zunger, in Electronic Structure of 3d Transition-Atom Impurities in Semiconductors, Vol. 39 of Solid State Physics, edited by H. Ehrenreich and D. Turnbull (Academic Press, New York, 1986).

[232] H. Lemke, Mater. Sci. Forum 196-201, 683 (1995). 
[233] H. Zimmermann and H. Ryssel, Appl. Phys. Lett. 58, 499 (1991).

[234] J.-U. Sachse, W. Jost, J. Weber, and H. Lemke, Appl. Phys. Lett. 71, 1379 (1997).

[235] W. Schröter and M. Seibt, in Deep Levels of Metal Impurities in Silicon, EMIS data review series, in Properties of Silicon (in press).

[236] L. W. Song, X. D. Zhan, B. W. Benson, and G. D. Watkins, Phys. Rev. B 42, 5765 (1990).

[237] B. G. Svensson, B. Mohadjeri, A. Hallén, J. H. Svensson, and J. W. Corbett, Phys. Rev. B 43, $2292(1991)$.

[238] D. V. Lang, H. G. Grimmeiss, E. Meijer, and M. Jaros, Phys. Rev. B 22, 3917 (1980).

[239] J. M. Meese, J. W. Farmer, and C. D. Lamp, Phys. Rev. Lett. 14, 1286 (1983).

[240] G. D. Watkins and J. W. Corbett, Phys. Rev. 121, A1001 (1961).

[241] L. Wang, X. C. Yao, J. Zhou, and G. G. Qin, Phys. Rev. B 38, 13494 (1988).

[242] L. A. Hemstreet and J. O. Dimmock, Phys. Rev. B 20, 1527 (1979).

[243] L. A. Hemstreet, Phys. Rev. B 22, 4590 (1980).

[244] G. G. DeLeo, G. D. Watkins, and W. B. Fowler, Phys. Rev. B 25, 4972 (1982).

[245] G. G. DeLeo, G. D. Watkins, and W. B. Fowler, Phys. Rev. B 25, 4962 (1982).

[246] P. Pêcheur and G. Toussaint, Physica B\&C 116, 112 (1983).

[247] G. D. Watkins, G. G. DeLeo, and W. B. Fowler, Phys. Stat. Sol. B 116, 28 (1983).

[248] G. Picoli, A. Chomette, and M. Lannoo, Phys. Rev. B 30, 7138 (1984).

[249] U. Lindfelt and A. Zunger, J. Phys. C 17, 6047 (1984).

[250] J. L. A. Alves and J. R. Leite, Phys. Rev. B 30, 7284 (1984).

[251] H. Katayama-Yoshida and A. Zunger, Phys. Rev. B 31, 7877 (1985).

[252] A. Fazzio, M. J. Caldas, and A. Zunger, Phys. Rev. B 32, 934 (1985).

[253] C. Delerue, M. Lannoo, and G. Allan, Phys. Rev. B 39, 1669 (1989).

[254] F. Beeler, O. K. Andersen, and M. Scheffler, Phys. Rev. B 41, 1603 (1990).

[255] J. Dreyhsig, H.-E. Gumlich, and J. W. Allen, Phys. Rev. B 48, 15002 (1993).

[256] M. Lannoo, Phys. Rev. B 36, 9355 (1987).

[257] G. G. DeLeo, G. D. Watkins, and W. B. Fowler, Phys. Rev. B 37, 1013 (1988).

[258] S. Öğüt, H. Kim, and J. R. Chelikowsky, Phys. Rev. B 56, 11353 (1997).

[259] J. C. Slater, The Self-Consistent Field for Molecules and Solids (McGraw-Hill, New York, 1974), Vol. IV.

[260] J. F. Janak, Phys. Rev. B 18, 7165 (1978).

[261] G. A. Baraff and Schlüter, Phys. Rev. B 30, 3460 (1984).

[262] M. Stavola, private communication, 1999. 
[263] G. D. Watkins and J. W. Corbett, Phys. Rev. 134, A1359 (1964).

[264] A. N. Safonov, E. C. Lightowlers, G. Davies, P. Leary, R. Jones, and S. Öberg, Phys. Rev. Lett. 77, 4812 (1996).

[265] X. D. Zhan and G. D. Watkins, Phys. Rev. B 47, 6363 (1993).

[266] J.-U. Sachse, J. Weber, and H. Lemke, Mater. Sci. Forum 258-263, 307 (1997).

[267] M. J. Evans, M. Stavola, M. G. Weinstein, and S. J. Uftring, Mater. Sci. Eng. B (in press).

[268] P. M. Williams, G. D. Watkins, S. Uftring, and M. Stavola, Phys. Rev. Lett. 70, 3816 (1993).

[269] M. J. Evans, M. G. Gornstein, and M. Stavola, Mat. Res. Soc. Symp. Proc. 442, 275 (1997).

[270] S. J. Pearton, Appl. Phys. Lett. 40, 253 (1981).

[271] J. L. Benton, C. J. Doherty, S. D. Ferris, D. L. Flamm, L. C. Kimmerling, and H. J. Leamy, Appl. Phys. Lett. 36, 670 (1980).

[272] E. C. Lightowlers, Mater. Sci. Forum 196-201, 817 (1995).

[273] I. A. Veloarisoa, M. Stavola, D. M. Kozuch, and G. D. Watkins, Appl. Phys. Lett. 59, 2121 (1991).

[274] S. J. Uftring, M. Stavola, P. M. Williams, and G. D. Watkins, Phys. Rev. B 51, 9612 (1995).

[275] O. V. Feklisova and N. A. Yarykin, Semicond. Sci. Technol. 12, 742 (1997).

[276] R. Jones, J. Coomer, A. Resende, S. Öberg, and P. R. Briddon, (to be published).

[277] J. M. Kahn, L. M. Falicov, and E. E. Haller, Phys. Rev. Lett. 57, 2077 (1986).

[278] B. Clerjaud, D. Côte, and C. Naud, Phys. Rev. Lett. 58, 1755 (1987).

[279] A. Resende, R. Jones, S. Öberg, and P. R. Briddon, (to be published).

[280] J.-U. Sachse, Ph.D. thesis, Max-Planck-Institut für Festkörperforschung, Stuttgart, 1997.

[281] R. Jones, A. Resende, S. Öberg, and P. R. Briddon, Mater. Sci. Eng. B (in press).

[282] M. G. Weinstein and M. Stavola, (unpublished).

[283] S. M. Meyers, G. D. Petersen, and C. H. Seager, J. Appl. Phys. 80, 3717 (1996).

Contact FAX: +44-1392-264111, email: jones@excc.ex.ac.uk 